\title{
A determination of the charm content of the proton
}

\section{NNPDF Collaboration}

\author{
Richard D. Ball ${ }^{1}$, Valerio Bertone ${ }^{2}$, Marco Bonvini ${ }^{2}$, Stefano Carrazza ${ }^{3}$, Stefano Forte ${ }^{4, a}$ (i), Alberto Guffanti ${ }^{5}$, \\ Nathan P. Hartland ${ }^{2}$, Juan Rojo ${ }^{2}$, Luca Rottoli ${ }^{2}$ \\ ${ }^{1}$ The Higgs Centre for Theoretical Physics, University of Edinburgh, JCMB, KB, Mayfield Rd, Edinburgh EH9 3JZ, Scotland \\ ${ }^{2}$ Rudolf Peierls Centre for Theoretical Physics, University of Oxford, 1 Keble Road, Oxford OX1 3NP, UK \\ 3 Theory Division, CERN, Geneva, Switzerland \\ ${ }^{4}$ Dipartimento di Fisica, Università di Milano and INFN, Sezione di Milano, Via Veloria 16, 20133 Milan, Italy \\ ${ }^{5}$ Dipartimento di Fisica, Università di Torino and INFN, Sezione di Torino, Via P. Giuria 1, 10125 Turin, Italy
}

Received: 25 May 2016 / Accepted: 31 October 2016 / Published online: 24 November 2016

(C) The Author(s) 2016. This article is published with open access at Springerlink.com

\begin{abstract}
We present an unbiased determination of the charm content of the proton, in which the charm parton distribution function (PDF) is parametrized on the same footing as the light quarks and the gluon in a global PDF analysis. This determination relies on the NLO calculation of deepinelastic structure functions in the FONLL scheme, generalized to account for massive charm-initiated contributions. When the EMC charm structure function dataset is included, it is well described by the fit, and PDF uncertainties in the fitted charm PDF are significantly reduced. We then find that the fitted charm PDF vanishes within uncertainties at a scale $Q \sim 1.6 \mathrm{GeV}$ for all $x \lesssim 0.1$, independent of the value of $m_{c}$ used in the coefficient functions. We also find some evidence that the charm PDF at large $x \gtrsim 0.1$ and low scales does not vanish, but rather has an "intrinsic" component, very weakly scale dependent and almost independent of the value of $m_{c}$, carrying less than $1 \%$ of the total momentum of the proton. The uncertainties in all other PDFs are only slightly increased by the inclusion of fitted charm, while the dependence of these PDFs on $m_{c}$ is reduced. The increased stability with respect to $m_{c}$ persists at high scales and is the main implication of our results for LHC phenomenology. Our results show that if the EMC data are correct, then the usual approach in which charm is perturbatively generated leads to biased results for the charm PDF, though at small $x$ this bias could be reabsorbed if the uncertainty due to the charm mass and missing higher orders were included. We show that LHC data for processes, such as high $p_{T}$ and large rapidity charm pair production and $Z+c$ production, have the potential to confirm or disprove the implications of the EMC data.
\end{abstract}

\footnotetext{
a e-mail: stefano.forte@mi.infn.it
}

\section{Contents}

1 Introduction $\ldots \ldots \ldots \ldots \ldots \ldots$

2 Settings ................... 2

2.1 Experimental data . . . . . . . . . . . . 2

2.2 Theory. . . . . . . . . . . . . . . 3

2.3 Fit settings . . . . . . . . . . . . . . . . . 4

3 Results . . . . . . . . . . . . . 5 5

3.1 Fit results . . . . . . . . . . . 6

3.2 Dependence on the charm quark mass and fit stability 7

3.3 Impact of the EMC data . . . . . . . . . . . . 12

3.4 The charm PDF and its intrinsic component . . 15

4 LHC phenomenology . . . . . . . . . . . 21

4.1 Parton luminosities . . . . . . . . . . . . 21

4.2 LHC standard candles . . . . . . . . . . . . 21

4.2.1 Total cross sections . . . . . . . . . . 21

4.2.2 Differential distributions . . . . . . . 22

4.3 Probing charm at the LHC . . . . . . . 25

4.3.1 Z production in association with charm quarks 25

4.3.2 Charm quark pair production . . . . . . 28

5 Delivery and outlook . . . . . . . . . . . . . . . 29

References ... . . . . . . . . . . . . . 31

\section{Introduction}

Current general-purpose global PDF sets [1-7] assume that the charm PDF is perturbatively generated through pair production from gluons and light quarks. This assumption could be a limitation, and possibly a source of bias, for at least three different reasons. First, the charm PDF might have a nonvanishing "intrinsic" component of non-perturbative origin, such that it does not vanish at any scale within the perturba- 
tive region (see [8] for a recent review). Second, even if the charm PDF is purely perturbative in origin and thus vanishes below the physical threshold for its production, it is unclear what the value of this physical threshold is, as it is related to the charm pole mass, which in itself is not known very precisely. Finally, even if charm is entirely perturbative, and we knew accurately its production threshold, in practice massive charm production cross sections are only known at low perturbative order (at most NLO) and it is unclear whether this leads to sufficiently accurate predictions.

All these difficulties are solved if the charm quark PDF is parametrized and determined along with light quark and gluon PDFs. Whether or not the PDF vanishes, and, if it does, at which scale, will then be answered by the fit. From this point of view, the distinction between the perturbatively generated component, and a possible intrinsic component (claimed to be power suppressed [8,9] before mixing with other PDFs upon perturbative evolution) becomes irrelevant. This is quite advantageous because the ensuing PDF set automatically incorporates in the standard PDF uncertainty the theoretical uncertainty related to the size of the perturbative charm component due to uncertainty in the value of the charm mass. Also, the possible intrinsic component, though concentrated at large $x$ at a suitably chosen starting scale, will affect non-trivially PDFs at lower $x$ at higher scale due to mixing through perturbative evolution.

The aim of this paper is to perform a first determination of the charm PDF of the proton in which no assumption is made about its origin and shape, and charm is treated on the same footing as the other fitted PDFs. This will be done using the NNPDF methodology: we will present a variant of the NNPDF3.0 [1] PDF determination, in which the charm PDF is parametrized in the same way as the light quark and gluon PDFs, i.e. with an independent neural network with 37 free parameters. In the present analysis, we will assume the charm and anticharm PDFs to be equal, since there is currently not data which can constrain their difference.

The possibility of introducing a non-perturbative "intrinsic" charm PDF has been discussed several times in the past; see e.g. Refs. [10-15]. In all of these earlier studies, only charm PDFs with a restrictive parametrization based on model assumptions are considered. Moreover, in the CT family of PDF determinations $[11,13,15]$, intrinsic charm is introduced as a non-vanishing boundary condition to PDF evolution, but the massive corrections to the charm-initiated contributions $[16,17]$ are not included. While this would be consistent if all charm were generated perturbatively, as in the standard FONLL $[18,19]$ or S-ACOT [20] schemes, when there is a non-perturbative charm PDF it is justified only if this non-perturbative component is uniformly power-suppressed (of order $\Lambda^{2} / m_{c}^{2}$, as in Ref. [21]) over the full range of $x$.

Here, however, as explained above, we wish to be able to parametrize the charm PDF at any scale, without com- mitting ourselves to any specific hypothesis on its shape, and without having to separate the perturbative and nonperturbative components. A formalism which includes the mass corrections $[16,17]$ by extending the FONLL [18] GMVFN scheme for deep-inelastic scattering of Ref. [19] was implemented at NLO [22] and consistently worked out to all orders in [23]. It is this implementation that will be used in this paper.

In the present PDF fit we use essentially the same data as in the NNPDF3.0 PDF determination, including as before the HERA charm production cross-section combination [24], but extended to also include the EMC charm structure function data of Ref. [25], which is the only existing measurement of the charm structure function at large $x$. We also replace all the HERA inclusive structure function data with the final combined dataset [5].

The outline of the paper is the following. First, in Sect. 2 we present the settings of the analysis: the dataset we use, the NLO implementation of the theory of Refs. [22,23] for the inclusion of a fitted charm PDF, and the fit settings which have been used in the PDF fits.

In Sect. 3 we present the fit results: we compare PDF determinations with and without fitted charm; we discuss the stability of our results with respect to variations of the charm mass; and we discuss the features of our best-fit charm PDF, specifically in terms of the momentum fraction carried by charm, and in comparison to existing models. In Sect. 4 we discuss the implications of our results for LHC phenomenology, both for processes which are particularly sensitive to the charm PDF and thus might be used for its determination (such as $Z+c$ and charm pair production), and for LHC standard candles (such as $W, Z$ and Higgs production). Finally, in Sect. 5 we discuss the delivery of our results and outline future developments.

\section{Settings}

The PDF determination presented in this paper, which we will denote by NNPDF3IC, is based on settings which are similar to those used for the latest NNPDF3.0 global analysis [1], but with a number of differences, mostly related to the inclusion of a fitted charm PDF. These involve the experimental data, the theory calculations, and the fit settings, which we now discuss in turn.

\subsection{Experimental data}

The dataset used in the present analysis is the same as used for NNPDF3.0, with two differences. The first has to do with HERA data: for NNPDF3.0, the combined inclusive HERA-I data [26] were used along with the separate HERA-II datasets from the H1 and ZEUS Collaborations [27-30]. Meanwhile, 
the final HERA legacy combination [5] data have become available. These have been used here. It has been shown [31] that, while the impact of the HERA-II data on top of the HERA-I combined data is moderate but not-negligible, the impact of the global legacy combination in comparison to HERA-I and separate HERA-II measurements is extremely small. Nevertheless, this replacement is performed for general consistency. Similar conclusions on the impact of these data have been reached by the MMHT group [32].

The second difference is that we will also include EMC charm structure function data [25]. Since the EMC Collaboration presented this measurement in the early 1980 s, some studies $[10,12]$ have suggested that these data might provide direct evidence for non-perturbative charm in the proton $[8,33]$. On the other hand, some previous PDF fits with intrinsic charm have not been able to provide a satisfactory description of this dataset [14]. Since it is known that the EMC measurements were affected by some systematic uncertainties which were only identified after the experiment was completed, we will perform fits both with and without it. We will also perform fits where the EMC charm data have been rescaled to match the current value of the branching ratio of charm quarks into muons.

Summarizing, the dataset that we will use is the following: fixed-target neutral-current deep-inelastic scattering (DIS) data from NMC [34,35], BCDMS [36,37], SLAC [38] and EMC [25]; the legacy HERA combinations for inclusive [5] and charm [24] reduced cross sections; charged-current structure functions from CHORUS inclusive neutrino DIS [39] and from $\mathrm{NuTeV}$ dimuon production data $[40,41]$; fixedtarget E605 [42] and E866 [43-45] Drell-Yan production data; Tevatron collider data including the CDF [46] and D0 [47] $Z$ rapidity distributions and the CDF [48] onejet inclusive cross sections; LHC collider data including ATLAS [49-51], CMS [52-55] and LHCb [56,57] vector boson production, ATLAS [58,59] and CMS [60] jets, and finally, total cross-section measurements for top quark pair production data from ATLAS and CMS at 7 and $8 \mathrm{TeV}$ [6166]. Data with $Q<3.5 \mathrm{GeV}$ and $W^{2}<12.5 \mathrm{GeV}^{2}$ are excluded from the fit.

A final change in comparison to Ref. [1] is that we now impose additional cuts on the Drell-Yan fixed-target crosssection data:

$\tau \leq 0.08, \quad|y| / y_{\max } \leq 0.663$,

where $\tau=M^{2} / s$ and $y_{\max }=-(1 / 2) \log \tau$, and $y$ is the rapidity and $M$ the invariant mass of the dilepton pair. These cuts are meant to ensure that an unresummed perturbative fixed-order description is adequate; the choice of values is motivated by studies performed in Ref. [67] in relation to the determination of PDFs with threshold resummation, which turns out to have a rather larger impact on Drell-Yan pro- duction than on deep-inelastic scattering. These cuts reduce by about a factor 2 the number of fixed-target Drell-Yan data points included here in comparison to Ref. [1], and they improve the agreement between theory and data.

\subsection{Theory}

In the presence of fitted charm, the original FONLL expressions for deep-inelastic structure functions of Ref. [19] need to be modified to account for the new massive charm-initiated contributions [22,23]. Also, while in previous NNPDF determinations pole quark masses only have been used, here we will consider both pole and $\overline{\mathrm{MS}}$ heavy quark masses. These new features have been implemented along with a major update in the codes used to provide the theory calculations. Indeed, in all previous NNPDF determinations, PDF evolution and the computation of deep-inelastic structure functions were performed by means of the Mellin-space FKgenerator NNPDF internal code $[68,69]$. Here (and henceforth) we will use the public $x$-space APFEL code [70] for the solution of evolution equations and the computation of DIS structure functions. For hadronic observables, PDF evolution kernels are pre-convoluted with APPLgrid [71] partonic cross sections using the APFELCOmb interface [72].

The FKgenerator and APFEL codes have been extensively benchmarked. As an illustration, in Fig. 1 we show representative benchmark comparisons between deep-inelastic structure functions computed with the two codes. We plot the relative differences between the computation with either of these two codes of the inclusive neutral-current cross sections $\sigma_{\mathrm{NC}}\left(x, Q^{2}\right)$ at the $\mathrm{NMC}$ data points and for the charm production reduced cross sections $\sigma_{\mathrm{c} \overline{\mathrm{c}}}\left(x, Q^{2}\right)$ for the HERA data points. In each case we compare results obtained at LO (massless calculation) and using the FONLL-A, B and C general-mass schemes. Similar agreement is found for all other DIS experiments included in NNPDF3.0.

The agreement is always better than $1 \%$. Differences can be traced to the interpolation used by the FKgenerator, as demonstrated by the fact that they follow roughly the same pattern for all theoretical computations shown, with the largest differences observed for the NMC data, in the large $x$, low $Q^{2}$ region where the interpolation is most critical. Specifically, FKgenerator uses a fixed grid in $x$ with 25 points logarithmically spaced in $\left[x=10^{-5}, x=10^{-1}\right]$ and 25 points linearly spaced in $\left[x=10^{-1}, x=1\right]$, while APFEL instead optimises the distribution of the $x$-grid points experiment by experiment. Hence we estimate that with the current APFEL implementation accuracy has significantly improved to better than $1 \%$.

An advantage of using APFEL to compute DIS structure functions is that it allows for the use of either pole or $\overline{\mathrm{MS}}$ heavy quark masses $[73,74]$. The implementation of running masses in the PDF evolution in APFEL has been bench- 


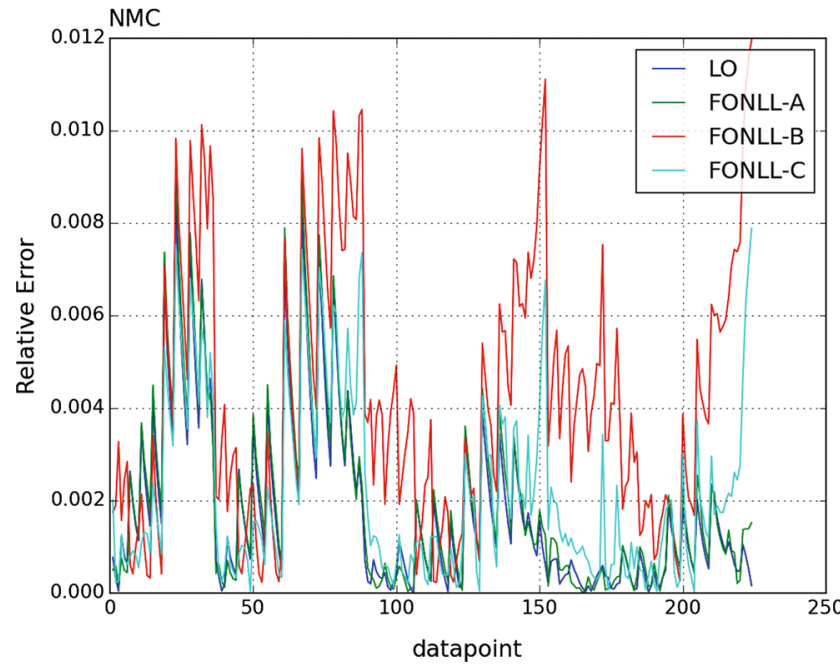

Fig. 1 Representative benchmark comparisons between deep-inelastic structure functions computed with the FKgenerator and APFEL programs. We show the relative differences between the two codes for $\sigma_{\mathrm{NC}}^{p}\left(x, Q^{2}\right)$ at the NMC data points (left) and for $\sigma_{\mathrm{c} \overline{\mathrm{c}}}\left(x, Q^{2}\right)$ for the

marked with the HOPPET program [75], finding better than $0.1 \%$ agreement. In addition, the APFEL calculation of structure functions with running heavy quark masses in the fixed three-flavour number scheme has been compared with the OpenQCDrad code [4], with which it has been found to agree at the $1 \%$ level.

Massive charm-initiated terms for both neutral and charged-current processes have been implemented in APFEL up to $\mathcal{O}\left(\alpha_{s}\right)$. Target mass corrections are included throughout. The implementation has been validated through benchmarking against the public stand-alone MassiveDISs Function code [76], which also implements the theory calculations of Refs. [22,23]. Some illustrative comparisons between the charm structure functions $F_{2}^{c}\left(x, Q^{2}\right)$ and $F_{L}^{c}\left(x, Q^{2}\right)$, computed using APFEL and MassivedISs Function, are shown in Fig. 2. The various inputs to the FONLL-A scheme computation, namely the three- and four-flavour scheme results are shown, along with the full matched result, as a function of $x$ at the scale $Q=5$ $\mathrm{GeV}$, computed using an input toy intrinsic charm PDF, corresponding to the NNPDF30_nlo_as_0118_IC5 set of Ref. [22]. The two codes turn out to agree at the $0.1 \%$ level or better, for all neutral-current and charged-current structure functions.

\subsection{Fit settings}

We can now specify the theory settings used for the PDF fits presented in this paper. We will use NLO theory with $\alpha_{s}\left(M_{Z}\right)=0.118$, with a bottom mass of $m_{b}=4.18 \mathrm{GeV}$. We will present fits with the $\overline{\mathrm{MS}}$ charm mass set equal to

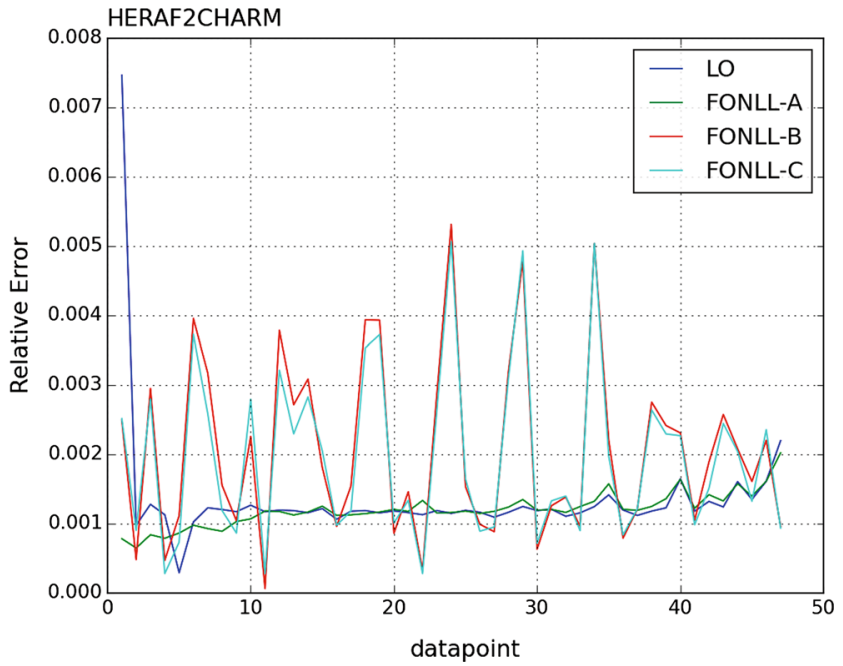

HERA charm data points (right). In each case, we show results at LO (massless calculation) and for the FONLL-A, B and C general-mass schemes

$m_{c}\left(m_{c}\right)=1.15,1.275$ and $1.40 \mathrm{GeV}$, which corresponds to the PDG central value and upper and lower five-sigma variations [77]. We will also present fits with the charm pole mass $m_{c}^{\text {pole }}=1.33,1.47$ and $1.61 \mathrm{GeV}$, obtained from the corresponding $\overline{\mathrm{MS}}$ values using one-loop conversion. This conservative range of charm pole mass value allows us to account for the large uncertainties in the one-loop conversion factor. In addition, as a cross-check, we also perform a pole mass fit with $m_{c}^{\text {pole }}=1.275 \mathrm{GeV}$, which was the choice adopted in NNPDF3.0. When the charm PDF is generated perturbatively, the charm threshold is set to be the charm mass. The input parametrization scale is $Q_{0}=1.1 \mathrm{GeV}$ for the fits with perturbative charm and $Q_{0}=1.65 \mathrm{GeV}$ in the case of fitted charm, ensuring that the scale where PDFs are parametrized is always above (below) the charm threshold for the analysis with fitted (perturbative) charm in all the range of charm masses considered. In sum, we will consider seven charm mass values (four pole, and three $\overline{\mathrm{MS}}$ ), and for each of them we will present fits with perturbative charm or with fitted charm.

In the NNPDF3.0 analysis, seven independent PDF combinations were parametrized with artificial neural networks at the input evolution scale $Q_{0}$ : the gluon, the total quark singlet $\Sigma$, the non-singlet quark triplet and octet $T_{3}$ and $T_{8}$ and the quark valence combinations $V, V_{3}$ and $V_{8}$. In this analysis, when we fit the charm PDF, we use the same PDF parametrization basis supplemented by the total charm PDF $c^{+}$, that is,

$c^{+}\left(x, Q_{0}\right) \equiv c\left(x, Q_{0}\right)+\bar{c}\left(x, Q_{0}\right)=x^{a c^{+}}(1-x)^{b_{c}+} \mathrm{NN}_{c^{+}}(x)$, 


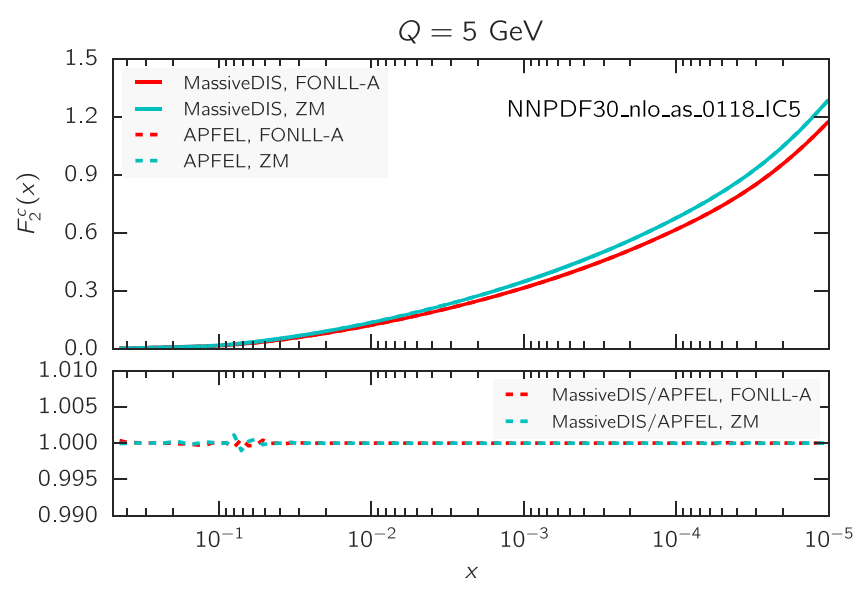

Fig. 2 Benchmarking of the implementation in the APFEL and MassiveDISsFunction codes of deep-inelastic structure functions in the FONLL-A scheme with intrinsic charm of Refs. [22,23]. The charm structure functions $F_{2}^{c}\left(x, Q^{2}\right)($ left $)$ and $F_{L}^{c}\left(x, Q^{2}\right)($ right $)$ are

with $\mathrm{NN}_{c^{+}}(x)$ a feed-forward neural network with the same architecture (2-5-3-1) and number of free parameters (37) as the other PDFs included in the fit, and $a_{c^{+}}$and $b_{c^{+}}$the corresponding preprocessing exponents, whose range is determined from an iterative procedure designed to ensure that the resulting PDFs are unbiased. In addition, we assume that the charm and anticharm PDFs are the same, $c^{-}\left(x, Q_{0}\right) \equiv$ $c\left(x, Q_{0}\right)-\bar{c}\left(x, Q_{0}\right)=0$. Since at NLO this distribution evolves multiplicatively, it will then vanish at all values of $Q^{2}$. It might be interesting to relax this assumption once data able to constrain $c^{-}\left(x, Q_{0}\right)$ become available.

The fitting methodology used in the present fits is the same as in NNPDF3.0, with some minor improvements. First, we have enlarged the set of positivity constraints. In NNPDF3.0, positivity was imposed for the up, down and strange structure functions, $F_{2}^{u}, F_{2}^{d}$ and $F_{2}^{s}$; for the light component of the longitudinal structure function, $F_{L}^{l}$; and for Drell-Yan rapidity distributions with the flavour quantum numbers of $u \bar{u}, d \bar{d}$, and $s \bar{s}$; and for the rapidity distribution for Higgs production in gluon fusion (see Section 3.2.3 of Ref. [1] for a detailed discussion). This set of positivity observables has now been enlarged to also include flavour non-diagonal combinations: we now impose the positivity of the $u d, \bar{u} d, \bar{u} \bar{d}$ and $u \bar{d}$ Drell-Yan rapidity distributions. As in Ref. [1], positivity is imposed for all replicas at $Q_{\text {pos }}^{2}=5 \mathrm{GeV}^{2}$, which ensures positivity for all higher scales.

Also, we have modified the way asymptotic exponents used in the iterative determination of the preprocessing range are computed. Specifically, we now use the definition

$$
\begin{aligned}
\alpha_{f_{i}}\left(x, Q^{2}\right) & \equiv \frac{\partial \ln \left[x f_{i}\left(x, Q^{2}\right)\right]}{\partial \ln x} \\
\beta_{f_{i}}\left(x, Q^{2}\right) & \equiv \frac{\partial \ln \left[x f_{i}\left(x, Q^{2}\right)\right]}{\partial \ln (1-x)},
\end{aligned}
$$

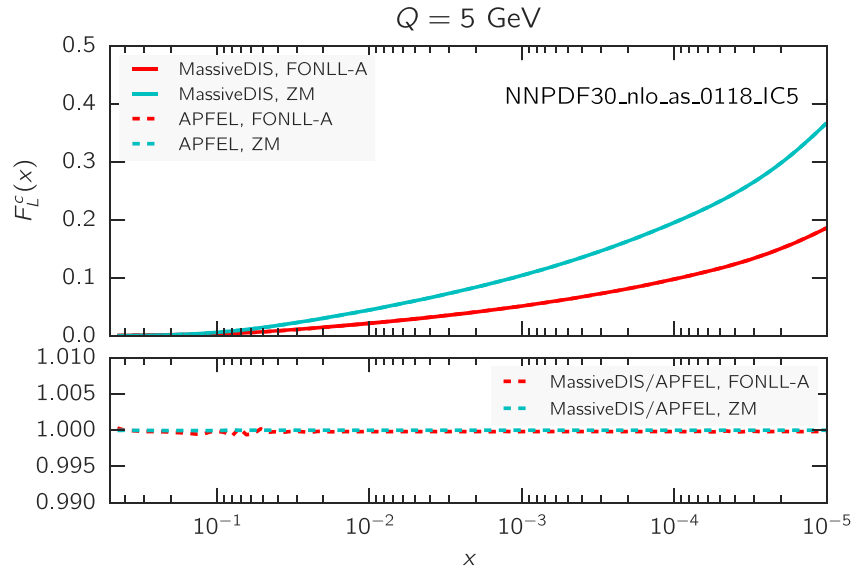

shown as a function of $x$ for $Q=5 \mathrm{GeV}$; the relative difference between the two codes is shown in the lower panel. In each case we show full matched FONLL-A results as well as the purely massless calculation

suggested in Refs. [78,79], which is less affected by subasymptotic terms at small and large- $x$ than the definition used in the NNPDF3.0 analysis [1]. This allows a more robust determination of the ranges in which the PDF preprocessing exponents should be varied, following the iterative procedure discussed in [1]. This modification affects only the PDFs in the extrapolation regions where there are little or none experimental data constraints available. The implications of these modifications in the global analysis will be more extensively discussed in a forthcoming publication.

\section{Results}

In this section we discuss the main results of this paper, namely the NNPDF3 PDF sets with fitted charm. After presenting and discussing the statistical indicators of the fit quality, we discuss the most significant effects of fitted charm, namely, its impact on the dependence of PDFs on the charm mass, and its effect on PDF uncertainties. We then discuss the extent to which our results are affected by the inclusion of EMC data on the charm structure function. Having established the robustness of our results, we turn to a study of the properties of the fitted charm PDF: whether or not it has an intrinsic component, the size of the momentum fraction carried by it, and how it compares to some of the models for intrinsic charm constructed in the past.

Here and henceforth we will refer to a fit using the FONLL-B scheme of Ref. [19], in which all charm is generated perturbatively, both at fixed order and by PDF evolution, as "perturbative charm", while "fitted charm" refers to fit obtained using the theory reviewed in Sect. 2.2. Note that fitted charm includes a perturbative component, which 
grows above threshold until it eventually dominates: at high enough scales most charm is inevitably perturbative. However, close to threshold the non-perturbative input might still be important: in particular below threshold the perturbative charm vanishes by construction, whereas the fitted charm can still be non-zero (so-called "intrinsic" charm).

\subsection{Fit results}

In Tables 1 and 2 we collect the statistical estimators for our best fit with central value of the charm pole mass, namely $m_{c}^{\text {pole }}=1.47 \mathrm{GeV}$, both with fitted and perturbative charm. A detailed discussion of statistical indicators and their meaning can be found in Refs. [1,69,80,81]. Here we merely recall that $\chi^{2}$ is computed by comparing the central (average) fit to the original experimental data; $\left\langle\chi^{2}\right\rangle_{\text {rep }}$ is computed by comparing each PDF replica to the data and averaging over replicas, while $\langle E\rangle$ is the quantity that is actually minimized, i.e. it coincides with the $\chi^{2}$ computed by comparing each replica to the data replica it is fitted to, with the two values given corresponding to the training and validation datasets, respectively. The values of $\langle E\rangle$ are computed using the so-called $t_{0}$ definition of the $\chi^{2}$, while for $\chi^{2}$ and $\left\langle\chi^{2}\right\rangle_{\text {rep }}$ we show in the table values computed using both the $t_{0}$ and the "experimental" definition (see Refs. [82,83] for a discussion of different $\chi^{2}$ definitions); they are seen to be quite close anyway.

Moreover, $\langle\mathrm{TL}\rangle$ is the training length, expressed in number of cycles (generations) of the genetic algorithm used for minimization. $\varphi_{\chi^{2}}[1]$ is the average over all data of uncertainties and correlations normalized to the corresponding experimental quantities (i.e., roughly speaking, $\varphi_{\chi^{2}}=0.5$ means that the PDF uncertainty is half the uncertainty in the original

Table 1 Statistical estimators of the fitted and perturbative charm PDFs for the central value of the charm pole mass, for both fitted charm and perturbative charm. For $\chi^{2}$ and $\left\langle\chi^{2}\right\rangle$ we provide the results using both the $t_{0}$ and the "experimental" definition of the $\chi^{2}$ (see text). $\left\langle E_{\mathrm{tr}}\right\rangle$ and $\left\langle E_{\mathrm{val}}\right\rangle$ are computed during the fit using the $t_{0}$ definition

NNPDF3 NLO $m_{c}=1.47 \mathrm{GeV}$ (pole mass)

\begin{tabular}{lll}
\hline & Fitted charm & Perturbative charm \\
\hline$\chi^{2} / N_{\text {dat }}(\exp )$ & 1.159 & 1.176 \\
$\left\langle\chi^{2}\right\rangle_{\text {rep }} / N_{\text {dat }}(\exp )$ & $1.40 \pm 0.24$ & $1.33 \pm 0.12$ \\
$\chi^{2} / N_{\text {dat }}\left(t_{0}\right)$ & 1.220 & 1.227 \\
$\left\langle\chi^{2}\right\rangle_{\text {rep }} / N_{\text {dat }}\left(t_{0}\right)$ & $1.47 \pm 0.26$ & $1.38 \pm 12$ \\
$\left\langle E_{\text {tr }}\right\rangle / N_{\text {dat }}$ & $2.38 \pm 0.29$ & $2.32 \pm 0.16$ \\
$\left\langle E_{\text {val }}\right\rangle / N_{\text {dat }}$ & $2.60 \pm 0.37$ & $2.48 \pm 0.16$ \\
$\langle$ TL $\rangle$ & $(3.5 \pm 0.8) \times 10^{3}$ & $(2.2 \pm 0.8) \times 10^{3}$ \\
$\varphi_{\chi^{2}}$ & $0.49 \pm 0.02$ & $0.40 \pm 0.01$ \\
$\left\langle\sigma^{(\exp )}\right\rangle_{\text {dat }}$ & $13.1 \%$ & $12.2 \%$ \\
$\left\langle\sigma^{(\text {fit })}\right\rangle_{\text {dat }}$ & $7.4 \%$ & $4.4 \%$ \\
\hline
\end{tabular}

Table 2 The $\chi^{2}$ per data point for the experiments included in the present analysis, computed using the experimental covariance matrix, comparing the results obtained with fitted charm with those of perturbative charm. We also provide the total $\chi^{2} / N_{\text {dat }}$ of the fit, as well as the number of data points per experiment. In the case of perturbative charm, we indicate the values of the fit without the EMC data, and show in brackets the $\chi^{2}$ of this experiment when included in the fit

\begin{tabular}{lrll}
\hline NNPDF3 NLO $m_{c}=1.47 \mathrm{GeV}$ (pole mass) \\
\hline Experiment & $N_{\text {dat }}$ & $\begin{array}{l}\chi^{2} / N_{\text {dat }} \\
\text { Fitted charm }\end{array}$ & $\begin{array}{l}\chi^{2} / N_{\text {dat }} \\
\text { Perturbative charm }\end{array}$ \\
\hline NMC & 325 & 1.36 & 1.34 \\
SLAC & 67 & 1.21 & 1.32 \\
BCDMS & 581 & 1.28 & 1.29 \\
CHORUS & 832 & 1.07 & 1.11 \\
NuTeV & 76 & 0.62 & 0.62 \\
EMC & 16 & 1.09 & {$[7.3]$} \\
HERA inclusive & 1145 & 1.17 & 1.19 \\
HERA charm & 47 & 1.14 & 1.09 \\
DY E605 & 104 & 0.82 & 0.84 \\
DY E866 & 85 & 1.04 & 1.13 \\
CDF & 105 & 1.07 & 1.07 \\
D0 & 28 & 0.64 & 0.61 \\
ATLAS & 193 & 1.44 & 1.41 \\
CMS & 253 & 1.10 & 1.08 \\
LHCb & 19 & 0.87 & 0.83 \\
$\sigma(t \bar{t})$ & 6 & 0.96 & 0.99 \\
Total & 3866 & 1.159 & 1.176 \\
\hline & & &
\end{tabular}

data), while $\left\langle\sigma^{(\mathrm{exp})}\right\rangle_{\text {dat }}$ is the average percentage experimental uncertainty, and $\left\langle\sigma^{(\mathrm{fit})}\right\rangle_{\text {dat }}$ is the average percentage PDF uncertainty at data points.

In Table 2 we provide a breakdown of the $\chi^{2}$ per data point for all experiments (the value computed with the "experimental" definition only). In the case of perturbative charm, the $\chi^{2}$ values listed correspond to a fit without EMC data, with the $\chi^{2}$ for this experiment if it were included in the fit given in square parentheses. Note that the total $\chi^{2}$ values in this table are significantly lower than those reported in our previous global fit NNPDF3.0 [1]: this is mainly due the much lower $\chi^{2}$ value for HERA data, which in turn results from using the full combined HERA dataset instead of separate HERA-II H1 and ZEUS data.

It is clear from these comparisons that fitting charm has a moderate impact on the global fit: the fit is somewhat longer (by less than two sigma), and uncertainties on predictions are a little larger. However, the overall quality of the global fit is somewhat improved: at the level of individual experiments, in most cases the fit quality is similar, with the improvements in the case of fitted charm more marked for the HERA inclusive, SLAC, CHORUS and E866 data. The $\chi^{2} / N_{\text {dat }}$ of the HERA charm combination is essentially the same in the 

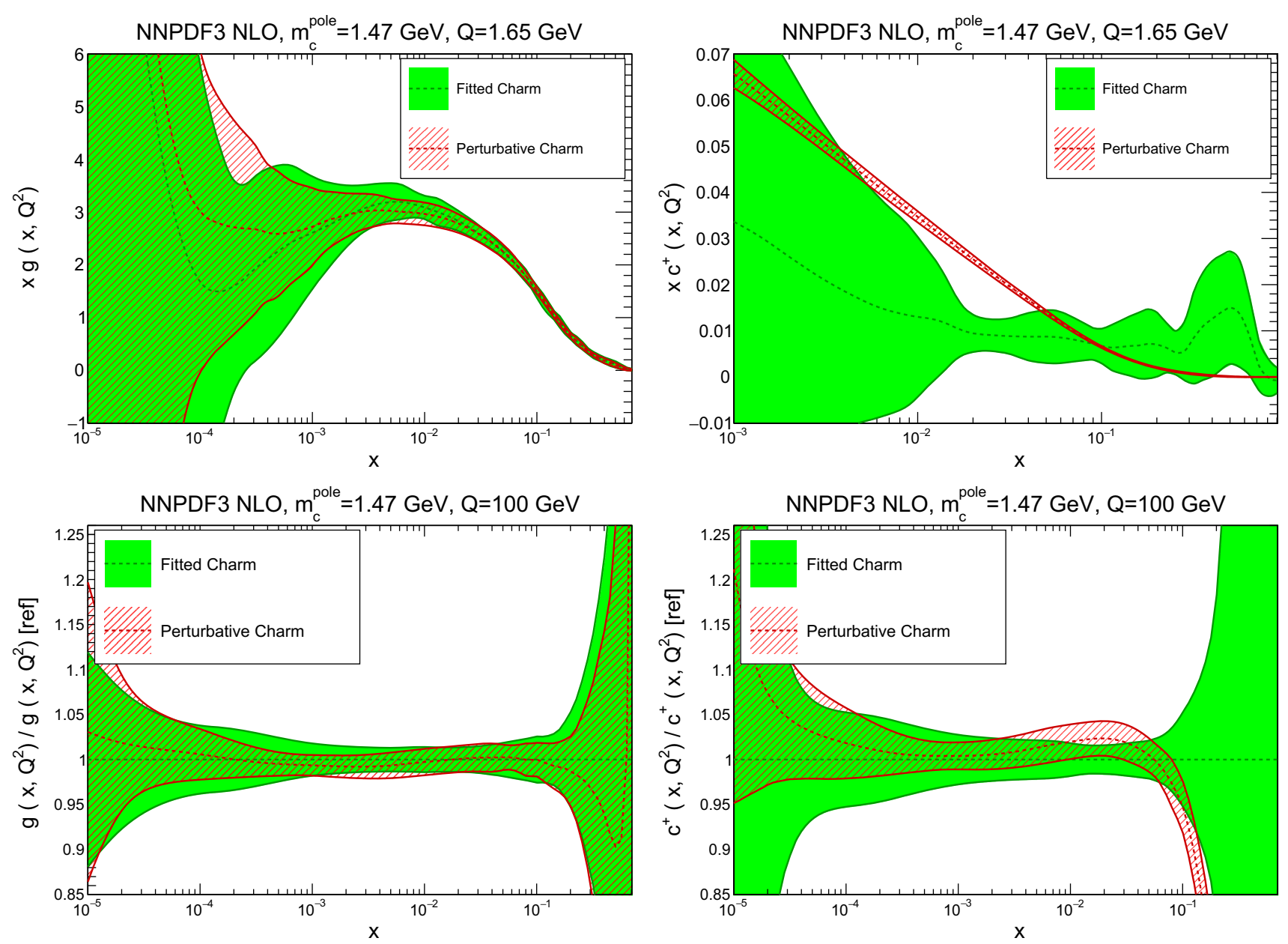

Fig. 3 Comparison of the NNPDF3 NLO PDFs with fitted and perturbative charm, for a charm pole mass of $m_{c}^{\text {pole }}=1.47 \mathrm{GeV}$. We show the gluon (left plots) and the charm quark (right plot), at a low scale

$Q=1.65 \mathrm{GeV}$ (upper plots) and at a high scale, $Q=100 \mathrm{GeV}$ (lower plots). In the latter case, results are shown normalized to the central value of the fitted charm PDFs

fitted and perturbative charm cases, and the fit quality to the LHC experiments is mostly unaffected, as expected since the measurements included have very limited direct sensitivity to the charm PDF.

On the other hand, the EMC charm structure function data cannot be fitted in a satisfactory way with perturbative charm: the best we can do without fitted charm is $\chi^{2} / N_{\text {dat }}=7.3$, corresponding to an increase in the total $\chi^{2}$ of over 100 units. However, the $\chi^{2}$ to these data improves dramatically when charm is fitted, and an excellent description with $\chi^{2} / N_{\mathrm{dat}}=1.09$ is achieved. It is interesting to note that some previous PDF determinations with intrinsic charm had difficulties in providing a satisfactory description of the EMC charm structure function data (see e.g. Ref. [14]). In the following, the EMC charm data will be excluded from the default fits with perturbative charm, though we will come back to the issue of including these data when charm is purely perturbative when discussing charm mass dependence in Sect. 3.2, and when specifically analyzing the impact of these data in Sect. 3.3.

In Figs. 3 and 4 we compare several PDFs with fitted and perturbative charm, both at a low scale, $Q=1.65 \mathrm{GeV}$ (just above the scale at which charm is generated in the purely perturbative fit), and at a high scale, $Q=100 \mathrm{GeV}$. It is clear that light quarks and especially the gluon are moderately affected by the inclusion of fitted charm, with a barely visible increase in the PDF uncertainty. The charm PDF and especially its uncertainty are affected more substantially: we will discuss this in detail in Sect. 3.4.

\subsection{Dependence on the charm quark mass and fit stability}

As discussed in the introduction, one of the motivations for introducing a fitted charm PDF is to separate the role of the charm mass as a physical parameter from its role in determining the boundary condition of the charm PDF. This dual role, 

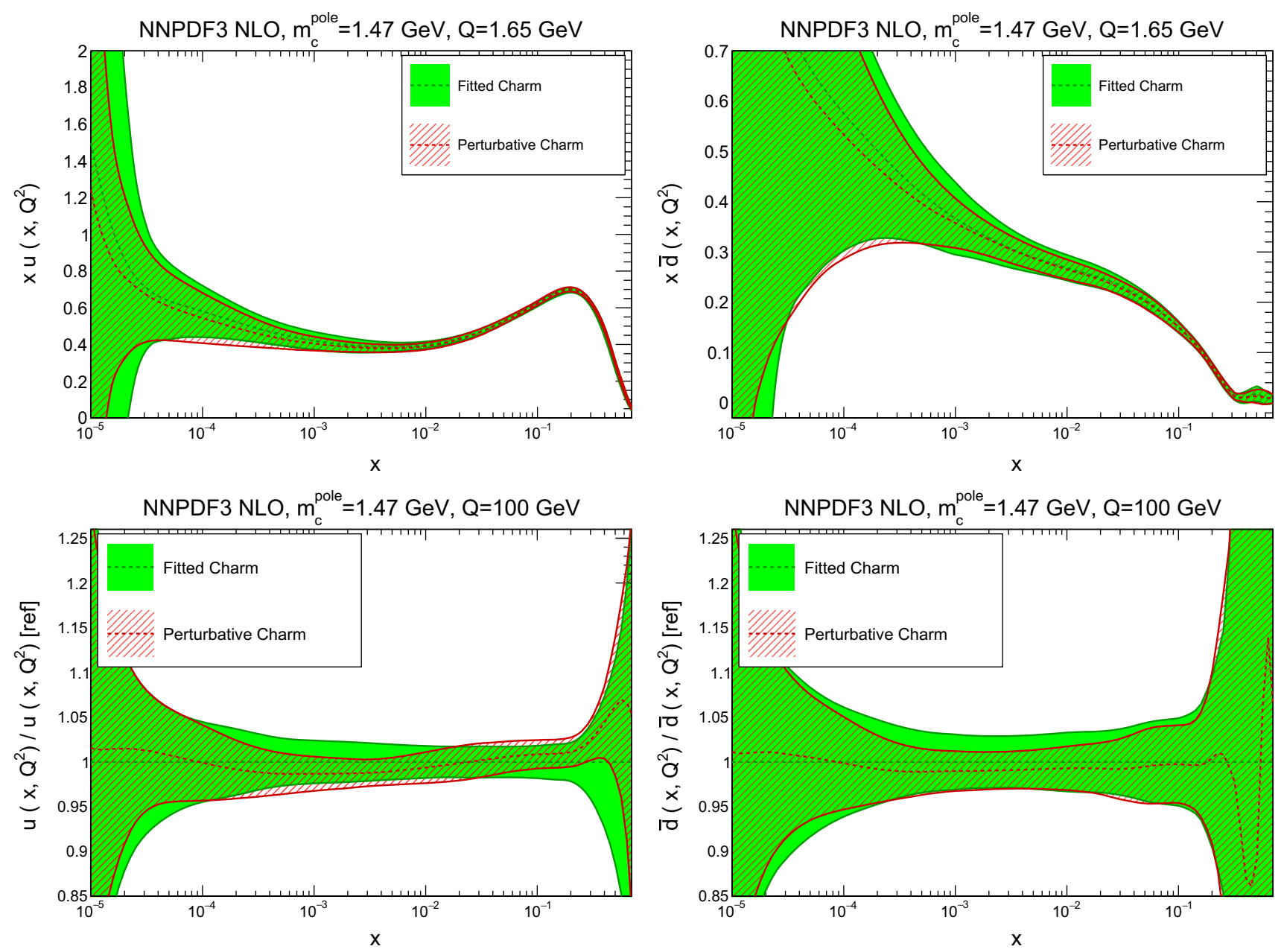

Fig. 4 Same as Fig. 3, but now showing the up (left) and antidown (right) PDFs

played by the charm mass, can be disentangled by studying the dependence of the fit results (and in particular the charm PDF) on the value of the charm mass when charm is perturbative or fitted. To this purpose, we compare fit results obtained when the charm mass is varied between $m_{c}^{\text {pole }}=1.33$ and $1.61 \mathrm{GeV}$ about our central $m_{c}^{\text {pole }}=1.47$, corresponding to a five-sigma variation in units of the PDG uncertainty on the $\overline{\mathrm{MS}}$ mass $m_{c}\left(m_{c}\right)$ using one-loop conversion to pole. After examining the stability of our results on the charm mass value, we discuss their stability with respect to different theoretical treatments. First, we show results for a fit with $m_{c}^{\text {pole }}=1.275 \mathrm{GeV}$, produced in order to compare with a fit with $\overline{\mathrm{MS}}$ masses with the same numerical value of $m_{c}$, and then we discuss how the fit results change if we switch from pole to $\overline{\mathrm{MS}}$ masses. Finally, we discuss how fit results would change if an S-ACOT-like treatment of the heavy quark was adopted, in which massive corrections to charm-initiated contributions are neglected.

For a first assessment of the relative fit quality, in Fig. 5 we show $\chi^{2} / N_{\text {dat }}$ as a function of the pole charm mass value, in the fits both with perturbative and fitted charm. The plot has been produced using the experimental definition of the $\chi^{2}$. The values shown here correspond to the full dataset, the inclusive and charm HERA structure function combined data, and EMC structure function data. In the case of perturbative charm, we generally show the results of a fit in which the EMC data are not included, except in the plot of the $\chi^{2}$ to the EMC data themselves, where we show both fits with EMC data included and not included. It is seen that the EMC data cannot be fitted when charm is perturbative in the sense that their poor $\chi^{2}$ does not significantly improve upon their inclusion in the fit. We will accordingly henceforth exclude the EMC data from all fits with perturbative charm, as their only possible effect would be to distort fit results without any significant effect on the fit quality.

It is interesting to observe that, while with fitted charm the EMC data seem to favour a value of the charm mass around $1.5 \mathrm{GeV}$, close to the current PDG average, with perturbative charm they would favour an unphysically 

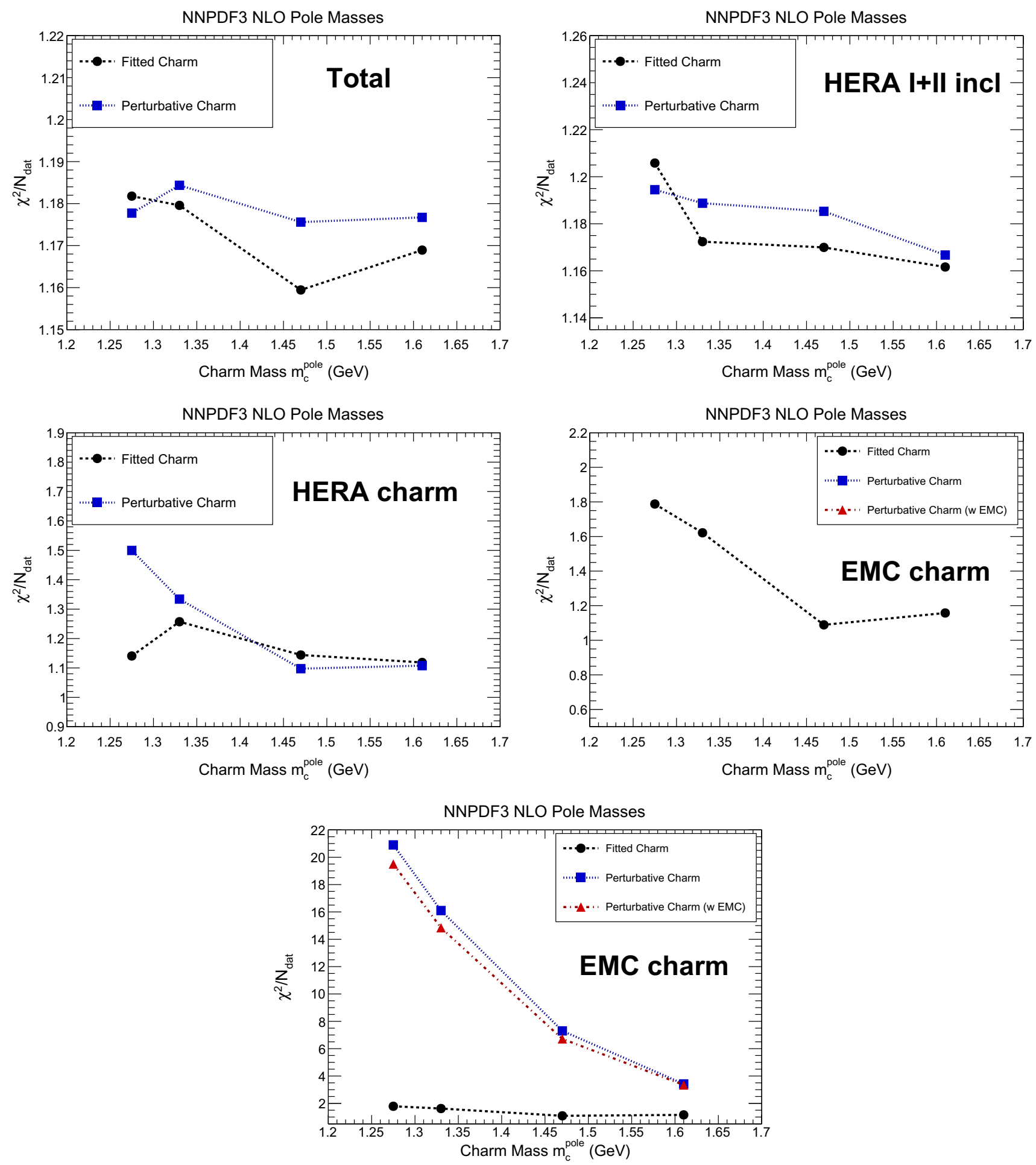

Fig. 5 The $\chi^{2}$ per data point for the total dataset (top left); for the HERA inclusive (top right) and charm structure function (center left) combined datasets and for the EMC charm data (center right), for fits with perturbative and fitted charm, as a function of the value of the charm pole mass $m_{c}^{\text {pole }}$. In the bottom row the $\chi^{2}$ for the EMC charm data is

large value. These results also suggest that a determination of the charm mass from a global fit with fitted charm might in principle be possible, but that this requires high shown again with an enlarged scale which enables the inclusion of the values for perturbative charm; in this plot only for fits with perturbative charm we show results both with and without the EMC data included in the fit. In all other plots, the perturbative charm results are for fits without EMC data. The fitted charm fits always include the EMC data

statistics and precision analysis techniques, such as those used in Refs. [84,85] for the determination of the strong coupling $\alpha_{s}$. 

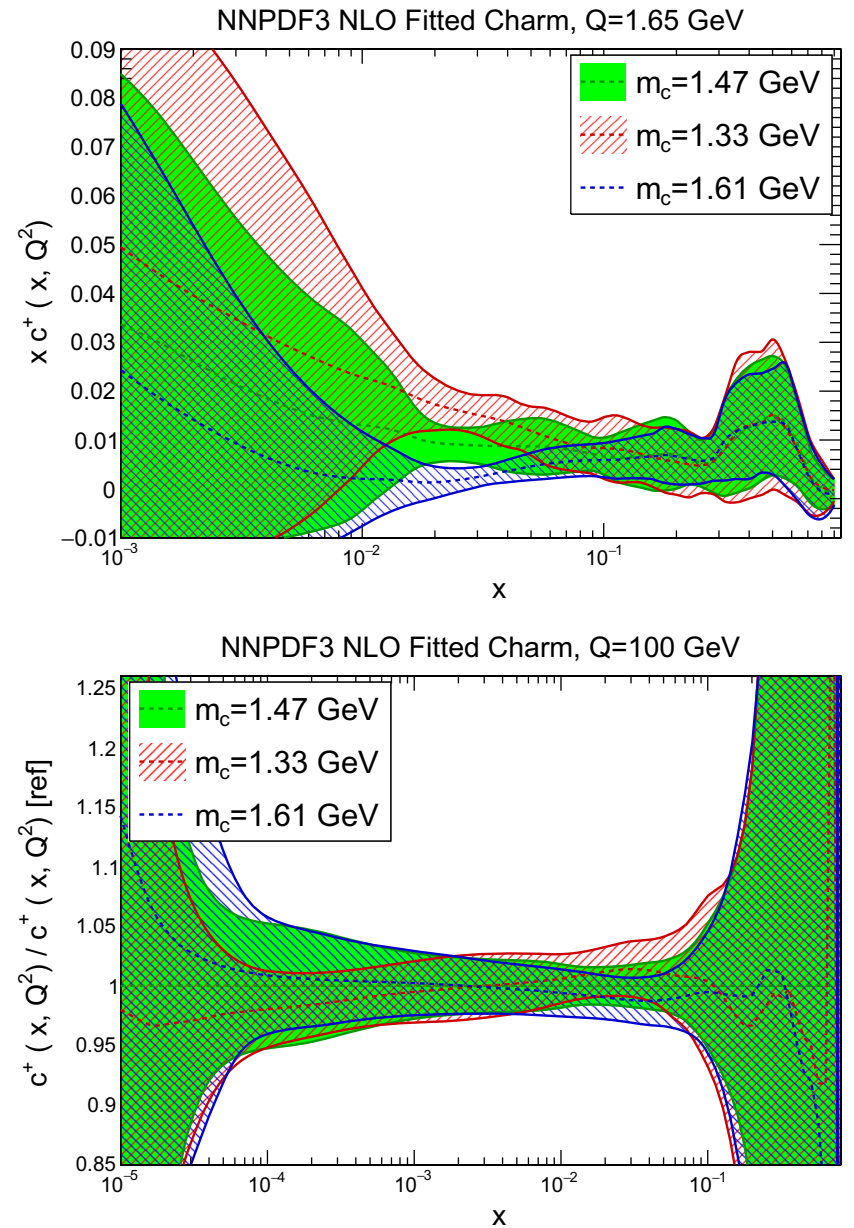

Fig. 6 Dependence of the charm PDF on the value of the pole charm mass $m_{c}^{\text {pole }}$ : the charm PDF obtained with fitted charm (left) and perturbative charm (right) are compared for $m_{c}^{\text {pole }}=1.33,1.47$ and 1.61

We now compare the PDFs obtained with different values of the charm mass both with perturbative and fitted charm: in Fig. 6 we show gluon and charm, and in Fig. 7 up and antidown quarks. Results are shown at low and high scale (respectively, $Q=1.65 \mathrm{GeV}$ and $Q=100 \mathrm{GeV}$ ) for charm, and at a high scale only for the light quarks. Of course, with perturbative charm the size of the charm PDF at any given scale depends significantly on the value of the charm mass that sets the evolution length: the lower the mass, the lower the starting scale, and the larger the charm PDF at any higher scale. The percentage shift of the PDF as the mass is varied is of course very large close to threshold, but it persists as a sizeable effect even at high scale. Remarkably, this dependence all but disappears when charm is fitted: both at low and high scale the fitted charm PDF is extremely stable as the charm mass is varied. This means that indeed once charm is fitted, its size is mostly determined by the data, rather than by the (possibly inaccurate) value at which we set the threshold for its production. Interestingly, the other PDFs, and specifically
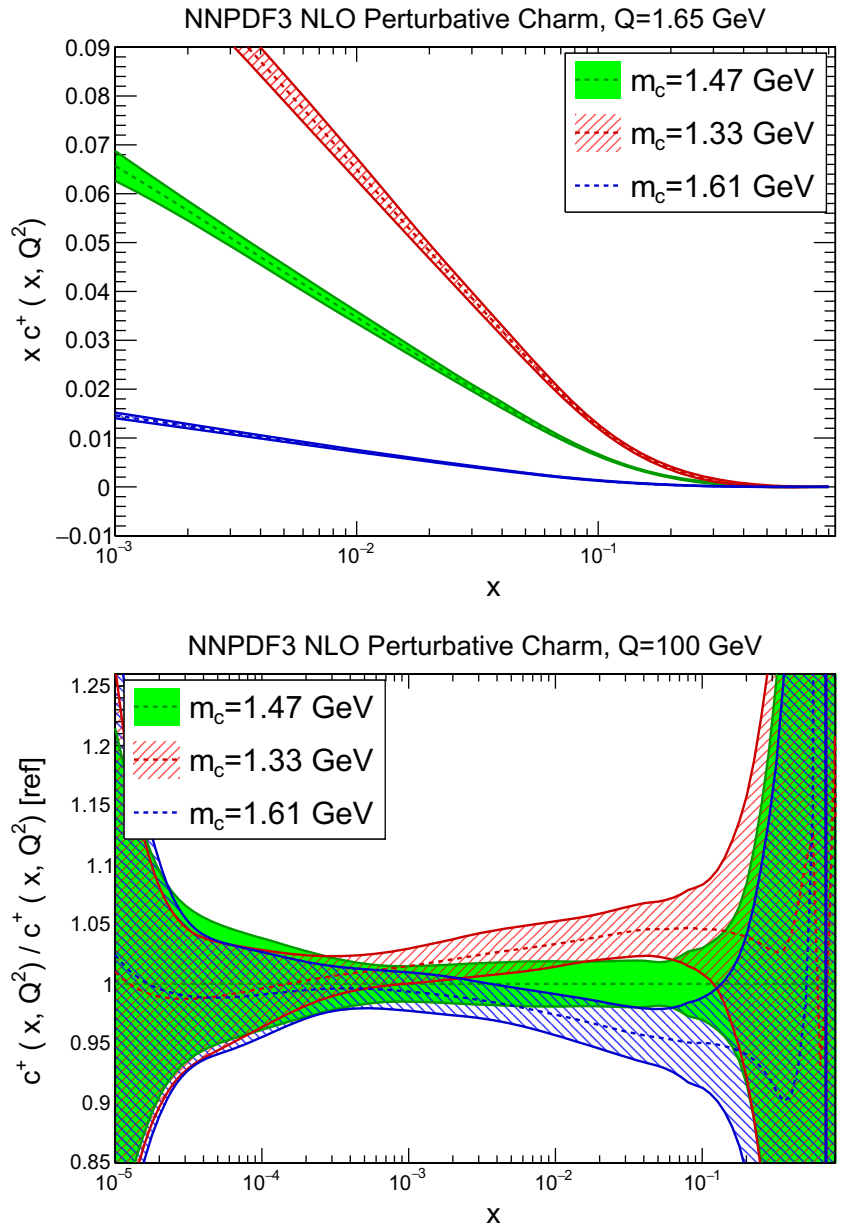

$\mathrm{GeV}$, at a low scale $Q=1.65 \mathrm{GeV}$ (top) and at a high scale $Q=100$ $\mathrm{GeV}$ (bottom). At high scale, PDFs are shown as a ratio to the fit with central $m_{c}^{\text {pole }}=1.47 \mathrm{GeV}$

the light quark PDFs, also become generally less dependent on the value of the heavy quark masses, even at high scale, thereby making LHC phenomenology somewhat more reliable.

This improved stability upon heavy quark mass variation can be seen in a more quantitative way by computing the pulls between the PDFs obtained using the two outer values of the charm mass, defined as

$$
\begin{aligned}
& P_{q}\left(x, Q^{2}\right) \\
& \quad \equiv \frac{\left.q\left(x, Q^{2}\right)\right|_{m_{c}=1.61 \mathrm{GeV}}-\left.q\left(x, Q^{2}\right)\right|_{m_{c}=1.33 \mathrm{GeV}}}{\left.\sigma_{q}\left(x, Q^{2}\right)\right|_{m_{c}=1.47 \mathrm{GeV}}}
\end{aligned}
$$

where $q$ stands for a generic PDF flavour, and $\sigma_{q}$ is the PDF uncertainty on the fit with the central $m_{c}$ value. The pull Eq. (4) evaluated at $Q=100 \mathrm{GeV}$ is plotted in Fig. 8 as a function of $x$ for the charm, gluon, down and antiup PDFs. It is clear that once charm is fitted the pull is essentially always less than one (that is, the PDF central value 

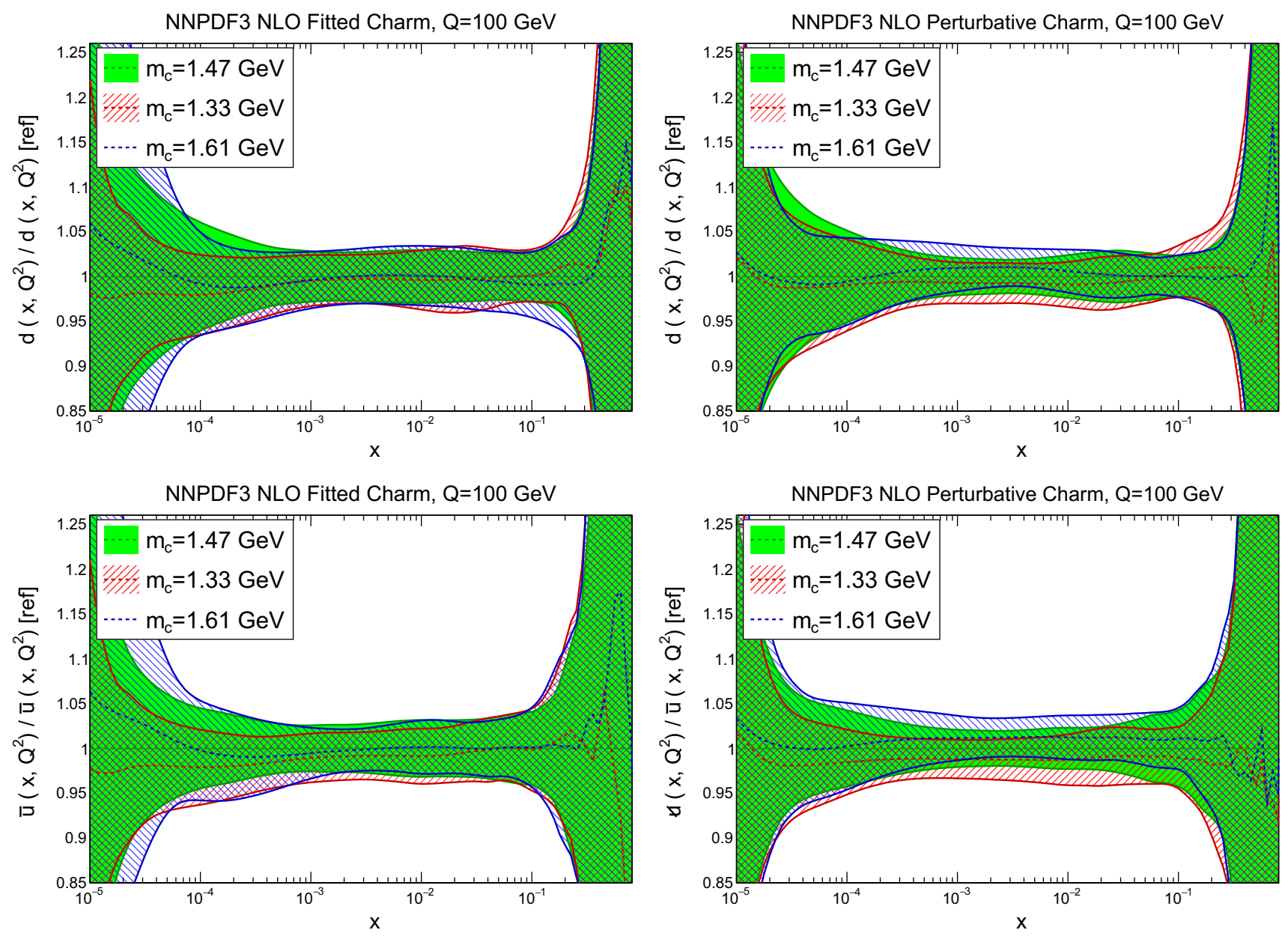

Fig. 7 Same as the bottom row of Fig. 6, but now for the down (top) and antiup (bottom) PDFs

varies by less than one sigma when the mass is varied in the given range), while it is somewhat larger for light quarks and gluon, and much larger (up to five sigma) for the charm PDF if charm is purely perturbative. The smallest difference is seen for the gluon, for which the pull is less than one in both cases, and in fact slightly larger for fitted charm when $x \sim 10^{-2}$.

We next check the impact of switching from pole to $\overline{\mathrm{MS}}$ masses. In Fig. 9 we compare PDFs obtained using pole mass $m_{c}^{\text {pole }}=1.47 \mathrm{GeV}$, or $\overline{\mathrm{MS}}$ mass $m_{c}\left(m_{c}\right)=1.275 \mathrm{GeV}$, the two values being related by one-loop perturbative conversion. The charm and gluon PDFs are shown, at low and high scale. It is clear that the change in results is compatible with a statistical fluctuation. Similar results hold for other PDFs.

Finally, we study how our results would change if massive charm-initiated contributions are neglected, i.e., if the original FONLL-B scheme of Ref. [19] is used. This corresponds to setting to zero the correction term $\Delta F_{h}$ (Eq. (11) of Ref. [22]), it is [22,23] completely equivalent to the S-ACOT scheme used in intrinsic charm studies by the CT Collabo- ration $[11,13,15]$, and, as mentioned in the introduction, it might be justified if the intrinsic charm contribution is powersuppressed. Results are shown in Fig. 10: again, the change in results is compatible with a statistical fluctuation. This fact has some interesting implications. First, it shows that the size our best-fit charm is moderate, and compatible with a powersuppressed intrinsic charm. Also, it suggests that the approximate NNLO treatment of fitted charm proposed in Ref. [22], in which these terms are actually only included up to NLO (given that the massive charm-initiated coefficient functions are only known to this order $[16,17])$, should actually be quite reliable. Finally, it should be noted that for the charminitiated contribution the charm production threshold is set by $m_{c}$, but for the overall process, including the proton remnant, the threshold is set by $2 m_{c}$, so there must be non-perturbative contributions which restore momentum conservation: these would appear as power-suppressed corrections which should be resummed to all orders when $W^{2} \sim m_{c}^{2}$. In our case $W^{2} \gg m_{c}^{2}$ for all $x$, and the charm-initiated contribution is seen to be sufficiently small, so that this issue should be of no concern. 


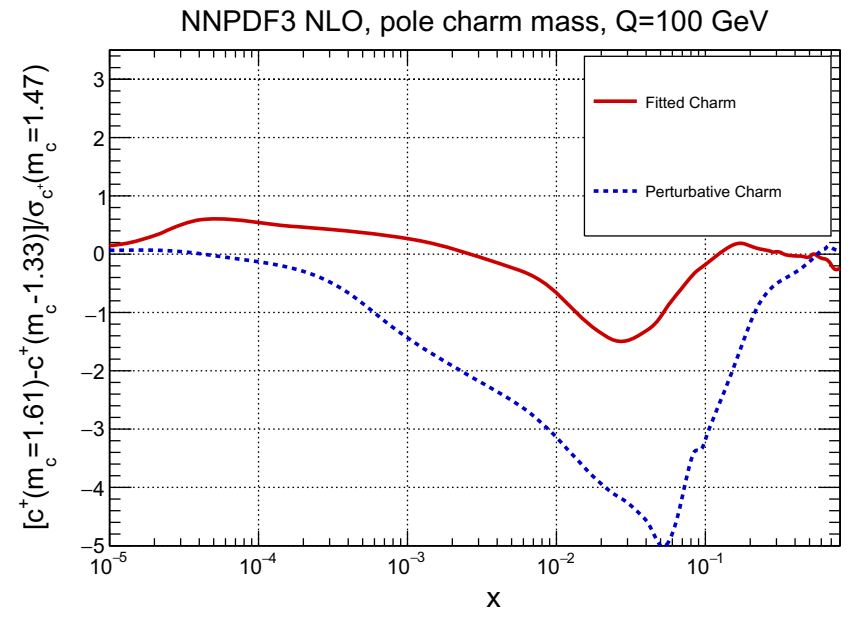

NNPDF3 NLO, pole charm mass, $Q=100 \mathrm{GeV}$

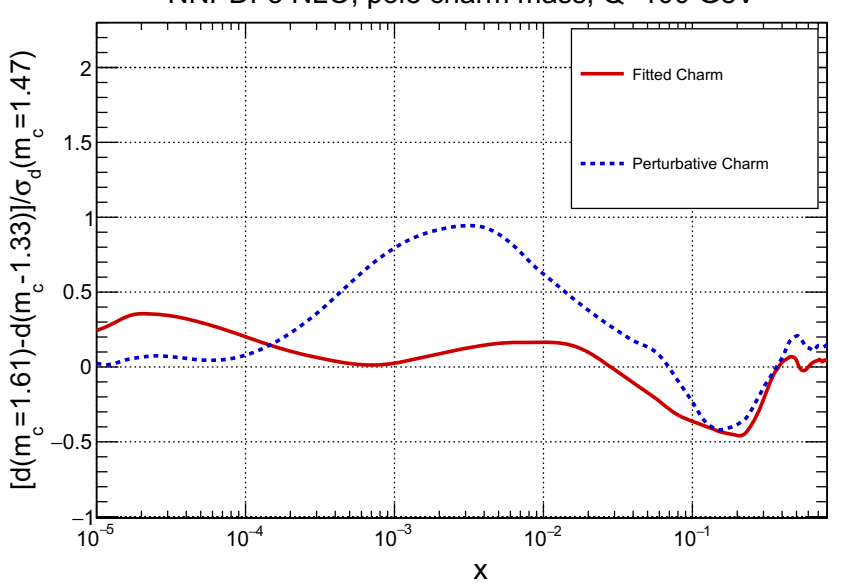

Fig. 8 The pull Eq. (4) between PDFs determined with the two outer values of the quark mass $\left(m_{c}=1.61 ; 1.33 \mathrm{GeV}\right)$, in units of the PDF uncertainty plotted as a function of $x$ at $Q=100 \mathrm{GeV}$. Results are

\subsection{Impact of the EMC data}

As already noted, it is not possible to fit the $\mathrm{EMC} F_{2}^{c}$ data of Ref. [25] with perturbative charm. It is then important to assess carefully the effect of these data when we fit charm. The purpose of this assessment is twofold. First, we have the phenomenological goal of assessing to which extent conclusions may be affected if the EMC data are entirely or in part unreliable, or perhaps have underestimated uncertainties. Second, perhaps more interestingly, we would like to understand whether, quite independently of the issue of their reliability, the EMC data might provide a realistic scenario in which not fitting charm would lead to biased fit results.

The agreement between data and theory when charm is fitted is illustrated in Fig. 11, where we compare the EMC charm structure function data with the structure function computed using the best-fit PDFs, with either fitted or per-

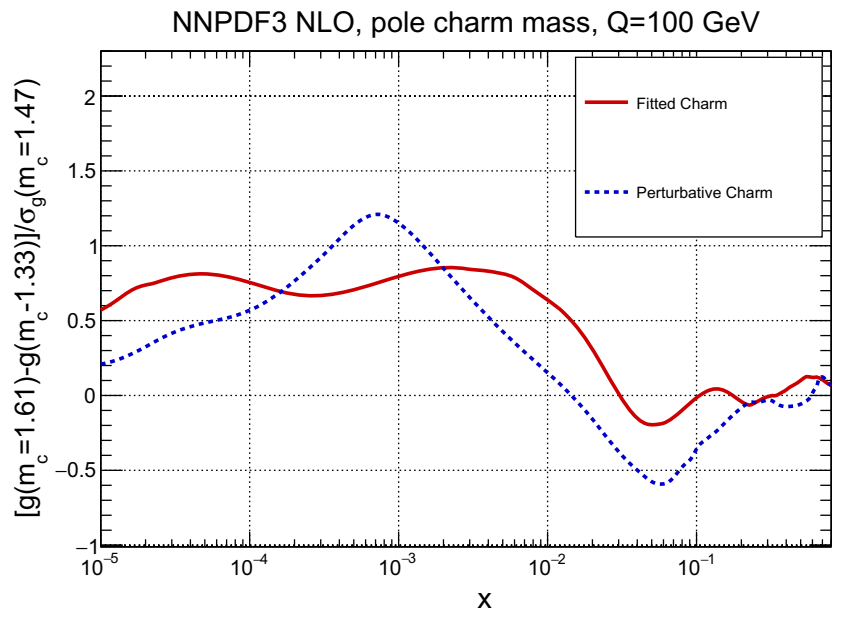

NNPDF3 NLO, pole charm mass, $Q=100 \mathrm{GeV}$

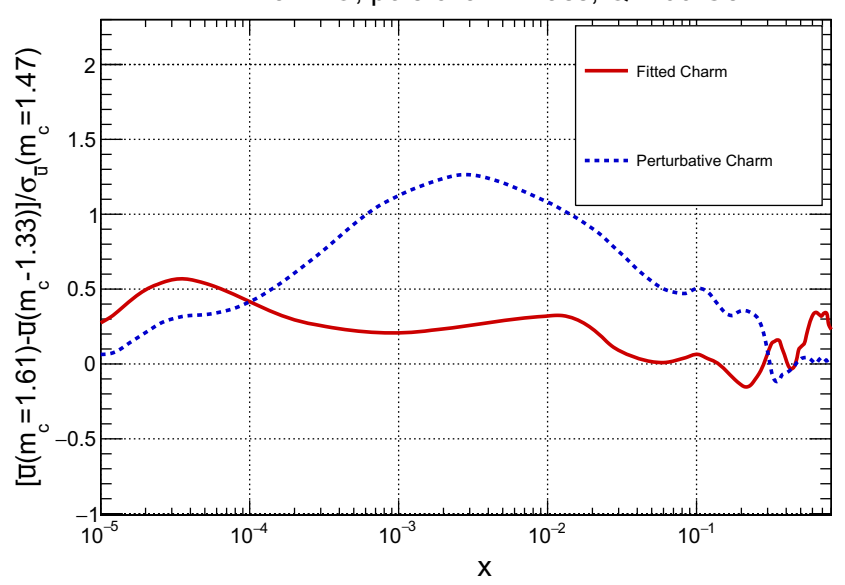

shown for charm (top left), gluon (top right), down (bottom left) and antiup (bottom right). Note the different scale on the $y$ axis in the different plots

turbative charm. Both the absolute structure function (top) and the theory to data ratio (bottom) are shown. It is interesting to observe that the discrepancy between the data and the perturbative charm PDFs is large, and it is not confined in any specific region of $x$ or $Q^{2}$, making an explanation of the discrepancy based on a single cause such as resummation or higher-order corrections rather unlikely. More specifically, it is clear that the data at large $x$ in the highest $Q^{2}$ bins cannot be reproduced by perturbative charm, which gives a very small contribution in this region. Interestingly, in this region one has $Q^{2} \gtrsim 25 \mathrm{GeV}^{2}$, so a possible higher-twist component that might imitate the charm contribution [86] would be quite suppressed. Likewise, in the small $x$ region, $x \lesssim 0.1$, perturbative charm overshoots the data. Here again, higher twist is expected to be small since, although $Q^{2}$ is quite low, $W^{2} \gtrsim 50 \mathrm{GeV}^{2}$. The fitted charm PDF corrects both these discrepancies rather neatly, by increasing the charm content at large $x$, and reducing it at small $x$, to produce 

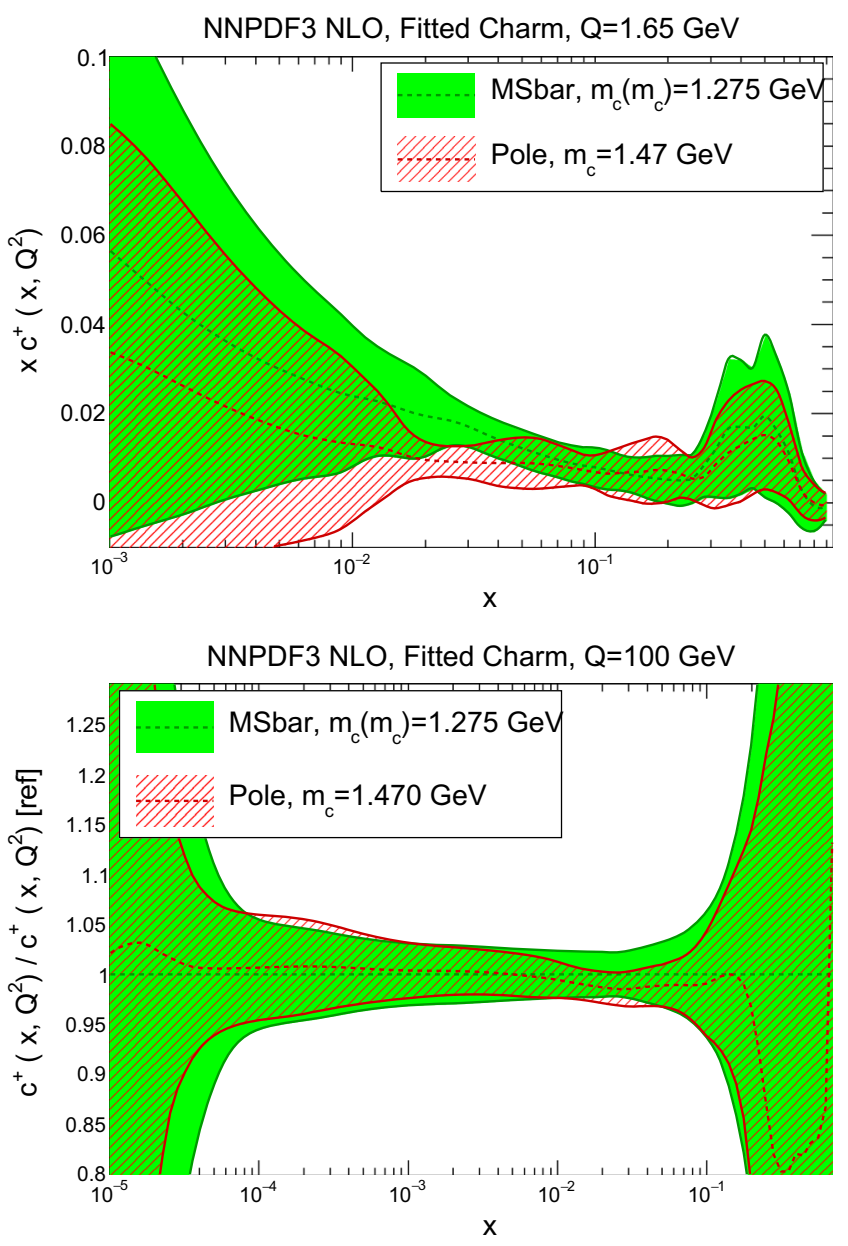

Fig. 9 Comparison of PDFs determined with $\overline{\mathrm{MS}}$ vs. pole mass, for corresponding values of the mass obtained by one-loop conversion: $m_{c}^{\text {pole }}=1.47 \mathrm{GeV}$ and $m_{c}\left(m_{c}\right)=1.275 \mathrm{GeV}$. The charm $($ left $)$ and

a perfectly satisfactory fit. This leads to the perhaps surprising conclusion that in order to fit the EMC data both a large- $x$ positive bump (possibly of non-perturbative origin), and a small $x$ undershoot (possibly mimicking missing higher-order corrections) are needed. Both the way the large $x$ behaviour of our best-fit charm compares to existing models and its small $x$ component compares to what we expect from missing higher orders will be discussed in Sect. 3.4 below.

The impact of the EMC data on the PDFs is illustrated in Fig. 12, where we compare the charm and gluon PDFs with and without the EMC data included in the fit, everything else being unchanged, with the perturbative charm fit also being shown for reference. It is clear that for all $x \gtrsim 10^{-2}$ the uncertainty on the fitted charm PDF is greatly increased in the absence of the EMC data. Reassuringly, the qualitative features of the central charm PDF (to be discussed more extensively in Sect. 3.4 below) do not change
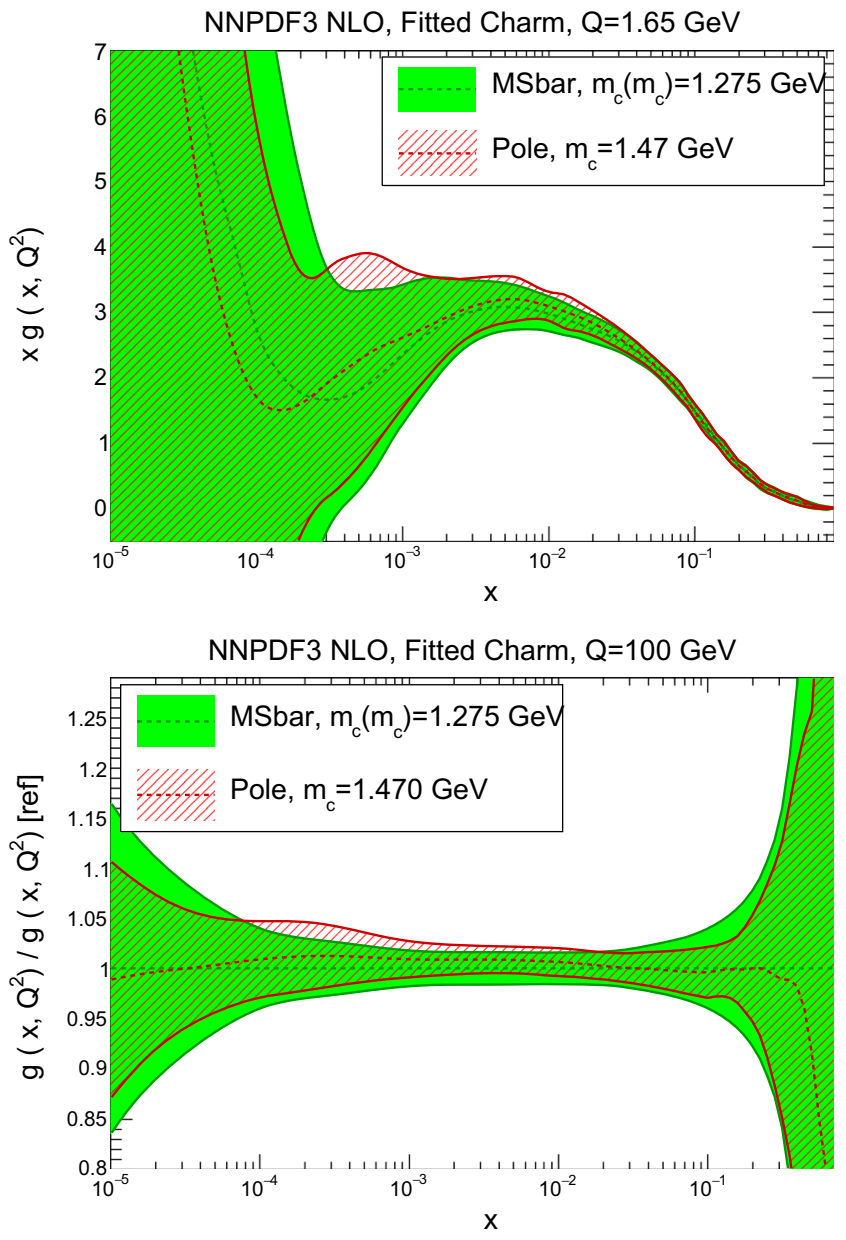

gluon (right) PDFs are shown, at low scale $Q=1.65 \mathrm{GeV}$ (top) and high scale $Q=100 \mathrm{GeV}$ (bottom). In the high-scale plots, results are shown as a ratio to the $\overline{\mathrm{MS}}$ mass result

substantially: in particular it is still true that the central PDF at large $x$ displays a bump, while at small $x$ it lies below the perturbatively generated charm-though uncertainties are now so large that neither effect can be considered statistically significant. The other PDFs change very little.

We now specifically address the phenomenological issue of the reliability of the EMC data. First of all, it should be noticed that the published uncertainty in the EMC data is quite large to begin with: the average uncertainty is about $27 \%$. This said, various issues have been raised concerning this dataset. Firstly, the inclusive EMC structure function data are known to be inconsistent with BCDMS data (see e.g. [87]), but this was due to underestimated backgrounds in drift chambers. Therefore, this problem is expected to be absent in the charm structure function data which were taken with a calorimetric target [88]. The correction is anyway never more than $20 \%$ [87], hence much smaller than the 

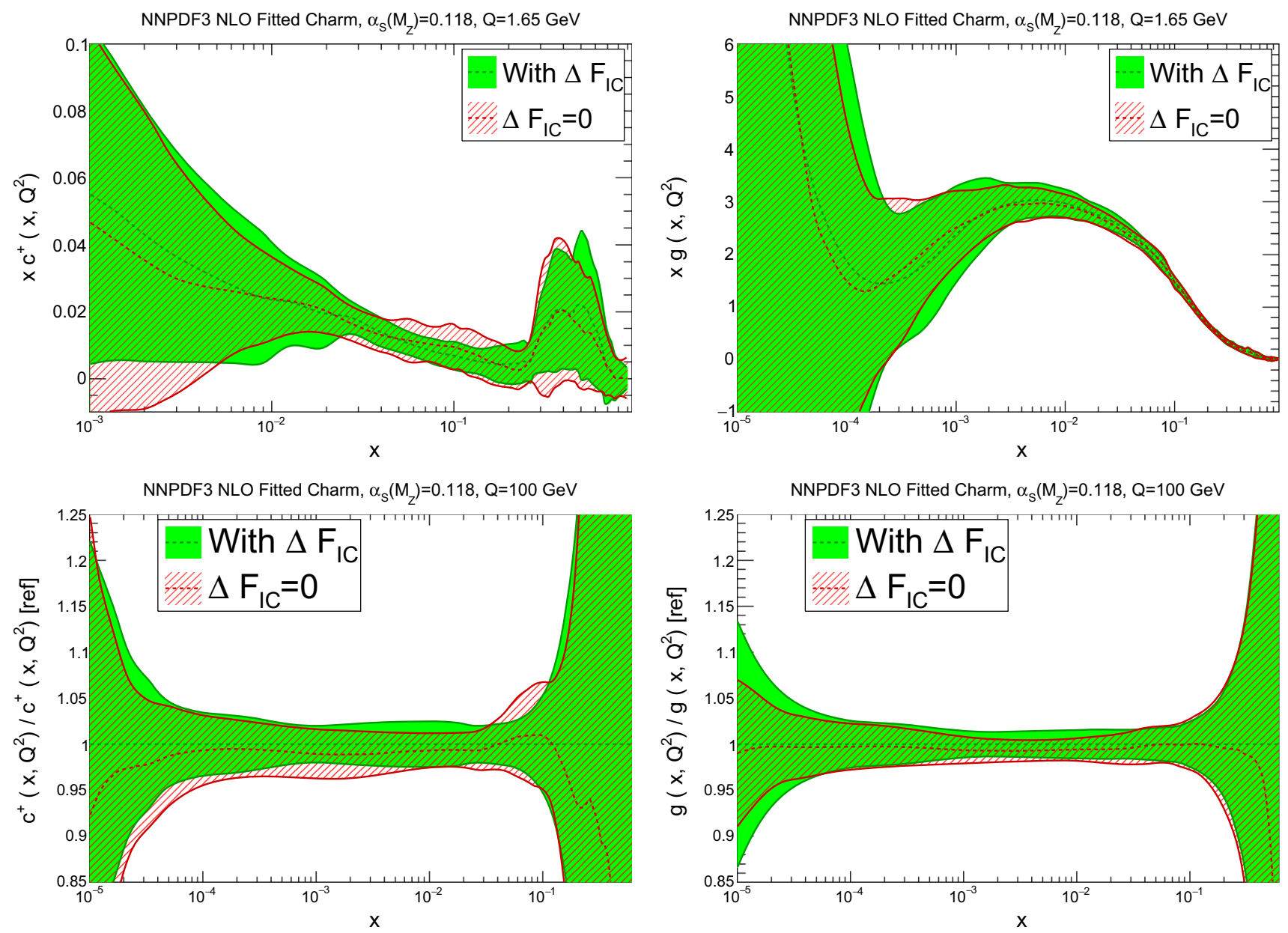

Fig. 10 Same as Fig. 9, but now comparing our default results to the case in which massive charm-initiated contributions are neglected (original FONLL-B of Ref. [19] or S-ACOT; see text)

effect seen in Fig. 11. In Ref. [89] it was checked explicitly that if the inclusive EMC data are added to the fit they have essentially no impact.

The original EMC charm structure functions were obtained assuming an inclusive branching fraction of $D$ mesons into muons, $\operatorname{BR}(D \rightarrow \mu+X)=8.2 \%$, which differs from the current PDG average [90] and the latest direct measurements from $\mathrm{LHCb}[91,92]$ of the fragmentation probabilities and branching fractions of $D$ mesons, which give a value of around $10 \%$. To verify the impact of using these updated branching fractions, and estimate also the possible impact of the other effects, we have rescaled the EMC data by a factor 0.82 and added an additional uncorrelated $15 \%$ systematic uncertainty due to $\operatorname{BR}(D \rightarrow \mu+X)$. The results are also shown in Fig. 12, where we see that this rescaling has only a small impact on the charm PDF. The impact becomes completely negligible if the systematics is taken to be correlated [89].

Since the charm data were taken on an iron target, nuclear corrections should be applied, as is the case also for the var- ious fixed-target neutrino datasets included in our global fit: in fact, in the smallest $x$ bins, shadowing corrections could be as large as $10-20 \%$ (see e.g. Ref. [93]). Furthermore, it was argued in Ref. [12] that higher-twist corrections obtained by replacing $m_{c}^{2}$ by $m_{c}^{2}\left(1+\frac{\Lambda^{2}}{m_{c}^{2}}\right)$ (where $\Lambda \sim 200 \mathrm{MeV}$ is a binding energy scale) may have a substantial effect on the lowest $Q^{2}$ (and thus smallest $x$ ) EMC data. Finally, of course, the EMC data have been obtained using analysis techniques which are quite crude to modern standards, for example only relying on LO QCD computations. The latter caveat, however, is in fact common to all the oldest fixed-target deep-inelastic scattering data which are still currently used for PDF determination, such as SLAC [38] and BCDMS [94,95], for which there is no evidence (see in particular Table 10 of Ref. [1]) that systematics are significantly underestimated, though, of course, specific issues only affecting EMC (such as the aforementioned background estimation) cannot be excluded.

In order to explore possible consequences of missing corrections (such as nuclear or higher twist), or uncertainty 

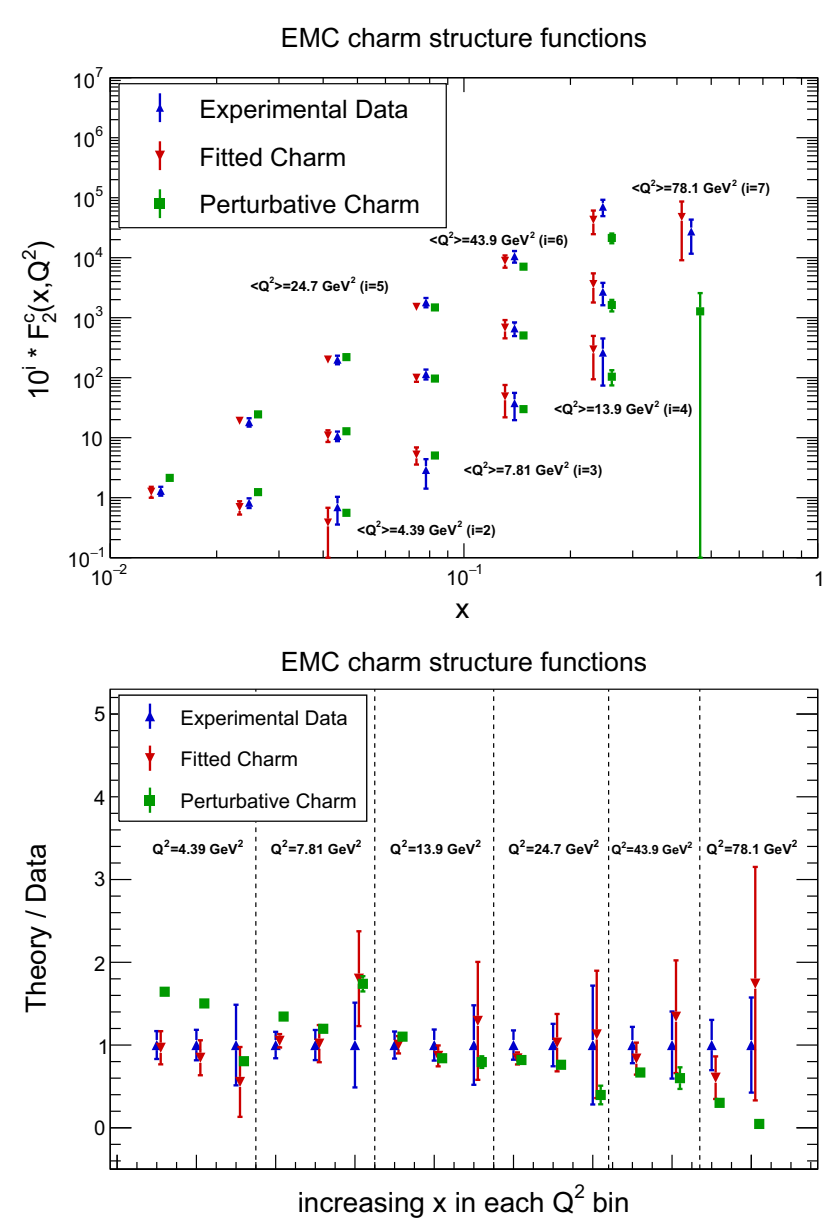

Fig. 11 Comparison of the best-fit theoretical result to the experimental result for the EMC $F_{2}^{c}$ structure function data with fitted and with perturbative charm. The uncertainties shown are the total PDF uncertainty in each data bin for theory, and the total experimental uncertainty for the data. We show both the data vs. $x$ in $Q^{2}$ bins, offset to improve readability (top), and the ratio of theory to data (bottom): here the order in each bin is from small to large values of $x$

underestimation, we have performed two more fits. In the first, we have removed all EMC data with $x<0.1$, namely the region where nuclear and higher-twist corrections are largest. In the second, we have retained all EMC data, but with an extra 50\% correlated systematics. Results are shown in Fig. 13. It is clear that the effect of the added systematics is minor: the percentage increase of uncertainties is moderate, and the central value changes very little. On the other hand, as one might expect, removing the small- $x$ EMC data leaves the best-fit charm unchanged for $x>0.1$, but for smaller $x$ it leads to results which are similar to those (shown in Fig. 12) when the EMC data are not included. This shows that the large $x$ EMC data are responsible for the large $x$ bump, while the small $x$ EMC data are responsible for the small $x$ undershoot in comparison to the perturbative charm case.
We conclude that, while we have no direct evidence that uncertainties in the EMC data might be underestimated, and specifically not more than for any other old deep-inelastic scattering dataset, there are persuasive theoretical arguments which suggest that these data might be affected by significant nuclear or higher-twist corrections, especially at small $x$. However, we find that even a very substantial increase of the systematic uncertainty of this data does not change its qualitative impact, as one might perhaps expect given the very large discrepancy between the data and predictions obtained with purely perturbative charm at small and large $x$. On the other hand, until more data are available phenomenological conclusions based on this data should be taken with a grain of salt, as is always the case when only a single dataset is responsible for a particular effect: as seen in Fig. 11, about half a dozen points are mostly responsible for the effect seen at small $x$ and as many at large $x$. However, regardless of the actual reliability of these data, there remains an issue of principle: if the EMC results were true, to what extent might the assumption of perturbative charm bias the fit result? This question is addressed in the next subsection.

\subsection{The charm PDF and its intrinsic component}

We now discuss the qualitative features of the best-fit charm PDF. Our goal here is not to assess the reliability of the data on which it is based (which was discussed in the previous subsection) but rather to examine the implication of a scenario in which such data are assumed to be true. Such a scenario does not appear to be forbidden or unphysical in any sense, so it is interesting to ask whether in this scenario a PDF determination without fitted charm would lead to biased results.

In order to get a first qualitative assessment, in Fig. 14 the charm PDF is plotted as a function of $x$ for various scales close to the threshold. Results are shown, for illustrative purposes, in the four-flavour scheme: in the three-flavour scheme the PDF would become scale independent. Both the fitted (left) and the perturbative (right) charm PDF are shown. The plot is produced from the fitted PDFs by backward evolution using APFEL from the scale $Q=1.65 \mathrm{GeV}$. Recall that the independence of the NNPDF results on the scale at which PDF are parametrized is a feature of the NNPDF approach which has been repeatedly verified; see e.g. Ref. [1].

The plot vs. $x$ on a logarithmic scale, in which the small $x$ region is emphasized, shows that for all $x \lesssim 10^{-1}$ the fitted charm lies below the perturbative charm. However, a scale $Q_{0}$ at which fitted charm vanishes for all $x$ in this region does appear to exist, but it is rather higher, around $Q_{0} \sim 1.6 \mathrm{GeV}$. Recalling that the dependence of the size of the charm PDF at small $x$ on the value of charm mass is very considerably reduced when charm is fitted (see Fig. 6), this is a genuine feature, which follows from the data. Of course, in the case 

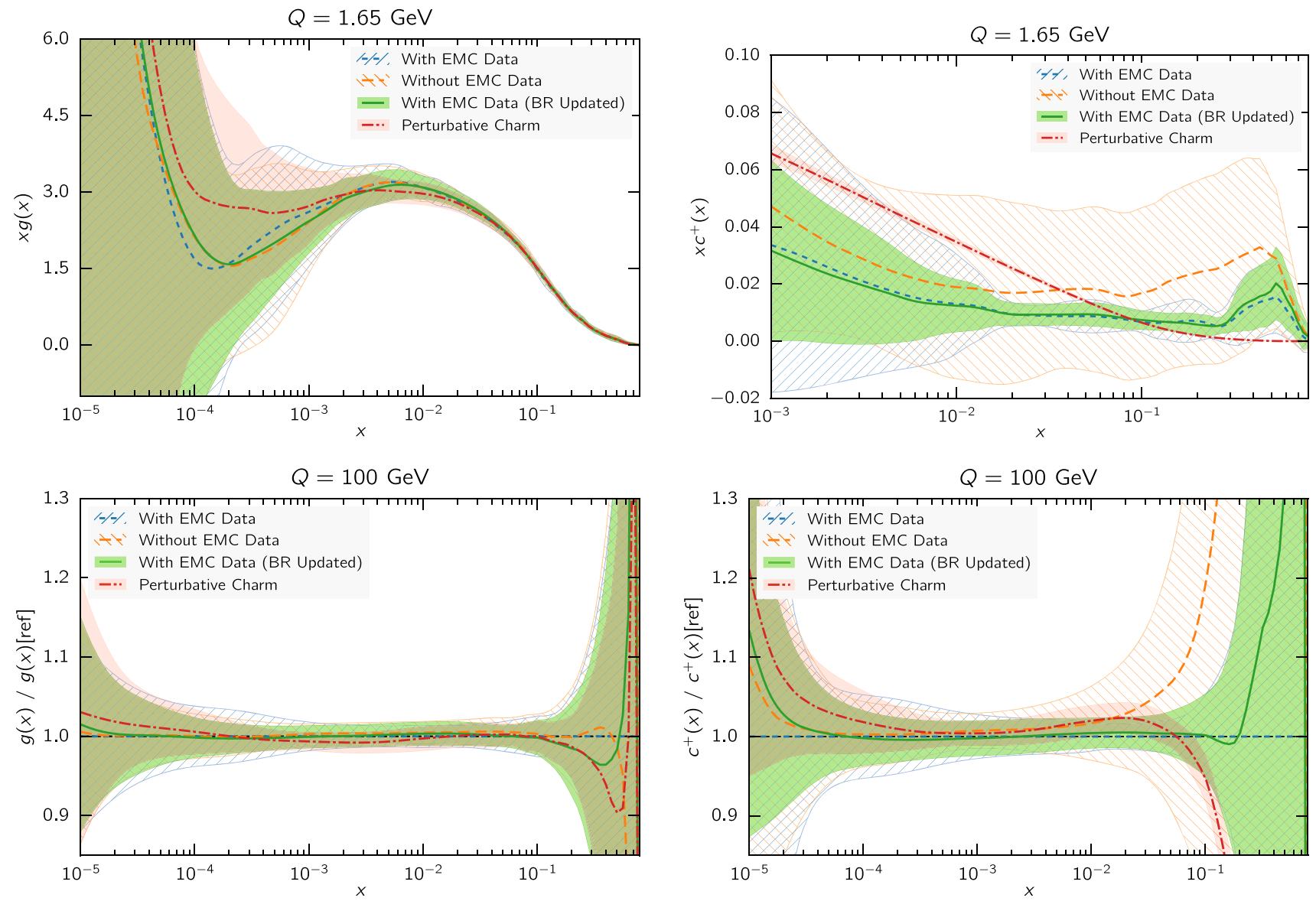

Fig. 12 Same as Fig. 3, but now, when charm is fitted, also showing results obtained when EMC data are rescaled to match updated branching fraction of $D$ mesons into muons (see text), or excluded altogether

of perturbative charm the scale at which the PDF vanishes is instead determined by the value of the mass, as is clear from the right plots of Fig. 14.

The plot vs. $x$ on a linear scale, in which the large $x$ region is emphasized, in turn shows that the fitted charm PDF displays an 'intrinsic' bump, peaked at $x \sim 0.5$ and very weakly scale dependent. This bump is of course absent when charm is generated perturbatively.

The impact of the EMC data on the features of the charm PDF shown in Fig. 14 can be traced to the behaviour shown in Fig. 11 and discussed in Sect. 3.3. Namely, at medium- $x$ and low- $Q^{2}$ the EMC data undershoot the prediction obtained using perturbative charm, while at large- $x$ and large $Q^{2}$ they overshoot it. This leads to a fitted charm which is significantly larger than the perturbative one at large $x$, but somewhat smaller at low $x$.

We now discuss each of these features in turn. To elucidate the small $x$ behaviour, in Fig. 15 we plot the charm PDF as a function of the scale $Q$ for fixed $x=0.01$, for the three values of the charm mass that have been considered above in Sect. 3.2. It is clear that, as mentioned, when charm is fitted (left) the scale at which the PDF vanishes is quite stable, while when charm is perturbative (right) the PDF is very sensitive to the value of the mass since the PDF is constrained to vanish at $Q=m_{c}$. Specifically the exact scale at which fitted charm vanishes at $x=0.01$ turns out to be $Q_{0}=1.59 \mathrm{GeV}$ (when $\left.m_{c}=1.47 \mathrm{GeV}\right)$.

In order to better understand the meaning of this result, in Fig. 16 we compare at the scale $Q=m_{c}=1.47$ the fitted charm to its perturbative counterpart determined at NLO and NNLO. While the NLO result vanishes by construction, the NNLO result (which will refer to as "NNLO perturbative charm" for short) is obtained using NNLO matching conditions [96,97] from our best-fit perturbative charm NLO PDF set. Within the FONLL-B accuracy of our calculation, this NNLO charm is subleading, hence it provides an estimate of the expected size of missing higher-order corrections on perturbatively generated charm.

It is interesting to observe that fitted charm for $x \lesssim 0.2$ is similar in size to NNLO perturbative charm, and it has in fact the same (negative) sign for $x \lesssim 0.02$. Of course, to the extent that fitted charm might reabsorb missing higher-order corrections, it would do so not only for matching terms but also for missing corrections to hard matrix elements, which 

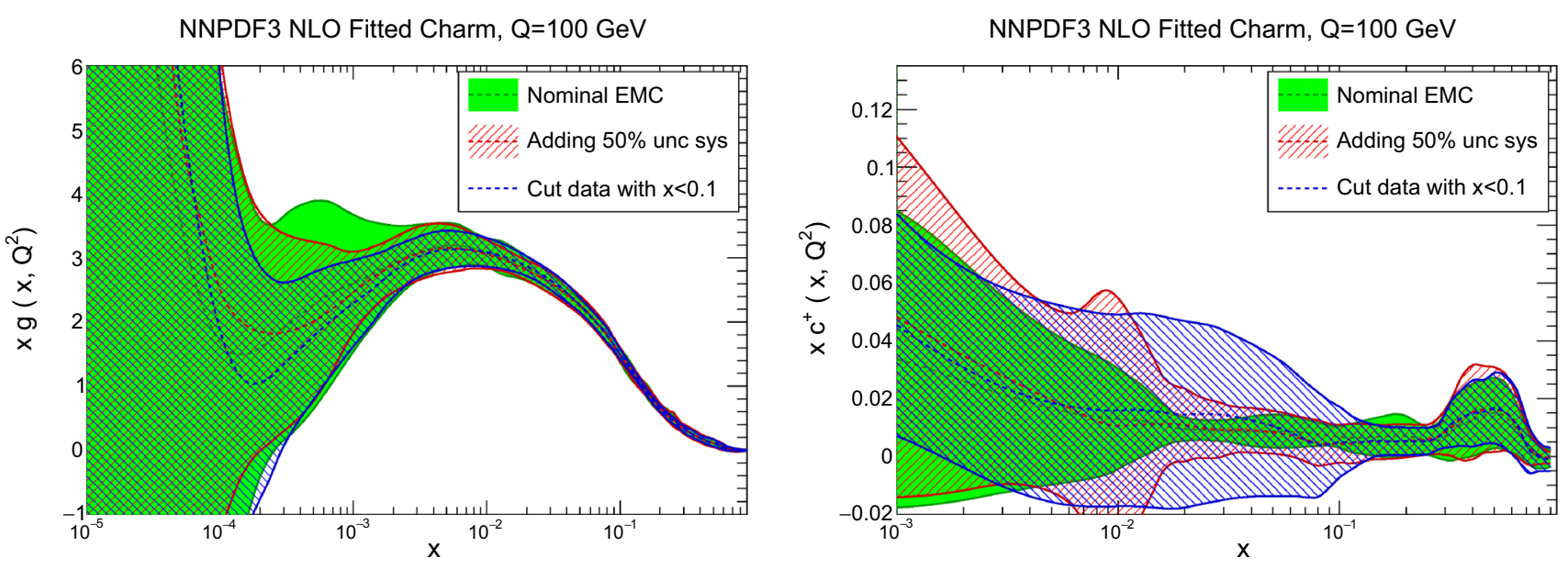

NNPDF3 NLO Fitted Charm, $Q=100 \mathrm{GeV}$
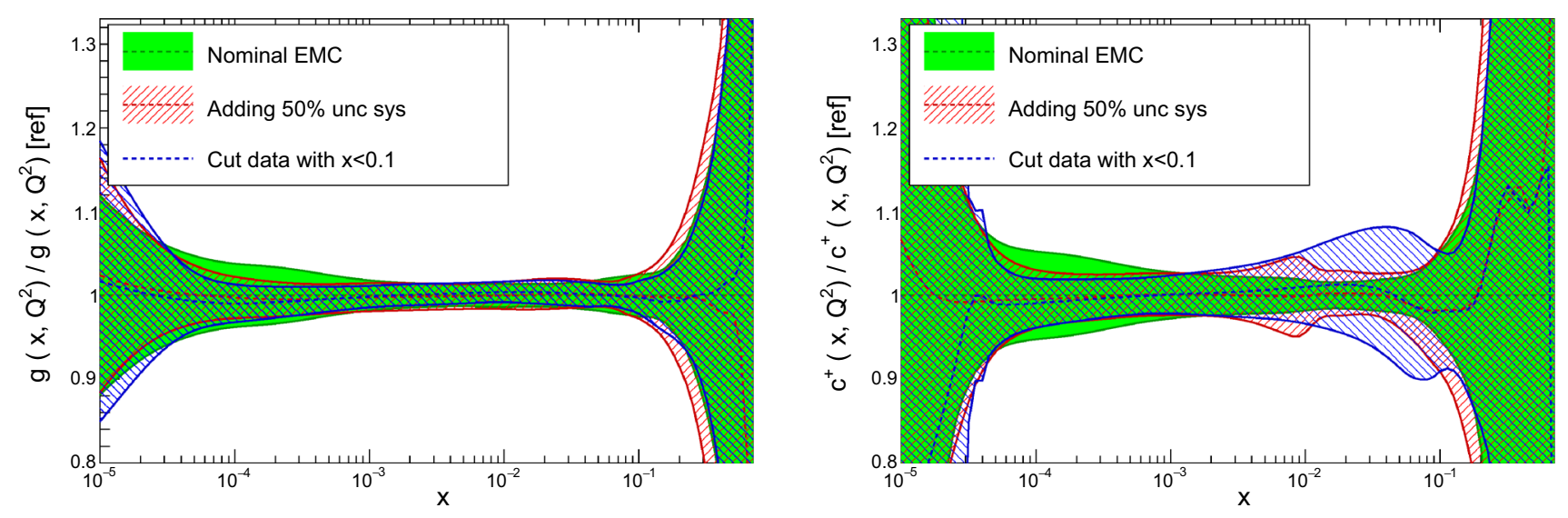

Fig. 13 Same as Fig. 3, but now comparing the default results with fitted charm with those obtained removing all EMC data with $x<0.1$, or adding and extra $50 \%$ systematics to all EMC data

are of the same order and likely of similar size. It is nevertheless intriguing that the observed undershoot of fitted charm when compared to perturbative charm is a feature of the NNLO matching condition at sufficiently small $x$.

All this suggests that our best-fit fitted charm at small $x$ is compatible with perturbative behaviour with either a somewhat larger value of the charm mass, or missing higher-order corrections reabsorbed into the initial PDF or a combination of both. This means that if uncertainties related to missing higher orders and the charm mass value were included in perturbative charm, then our fitted charm would be compatible with perturbative charm, but possibly more accurate (in view of the greater stability seen in Fig. 15 of the fitted charm in comparison to the perturbative one). If instead uncertainties related to missing higher orders and the charm mass value are not included (as it is now the case for most PDF sets, including NNPDF3.0) then the charm PDF, within the given uncertainty, is biased (assuming the EMC data are correct).

We now turn to the large $x$ behaviour. The fact that our fitted charm has an "intrinsic" component means that it car- ries a non-negligible fraction of the proton's momentum. In order to quantify this, we compute the momentum fraction carried by charm, defined as

$C\left(Q^{2}\right) \equiv \int_{0}^{1} \mathrm{~d} x x\left[c\left(x, Q^{2}\right)+\bar{c}\left(x, Q^{2}\right)\right]$

Of course for scales significantly above threshold, both the intrinsic and the perturbative components of the charm PDF will contribute. The momentum fraction $C\left(Q^{2}\right) \mathrm{Eq}$. (5) is plotted as a function of the scale $Q$ in Fig. 17, both for fitted and perturbative charm. In the case of fitted charm, results are shown both with and without the EMC data. In Fig. 18 we then show the momentum fraction with the three different values of the charm mass considered in Sect. 3.2.

The values of the momentum fraction at a low scale $Q=1.65 \mathrm{GeV}$ just above the charm mass, using the central value $m_{c}=1.47 \mathrm{GeV}$ are collected in Table 3: they show that both with and without the EMC data we find evidence 

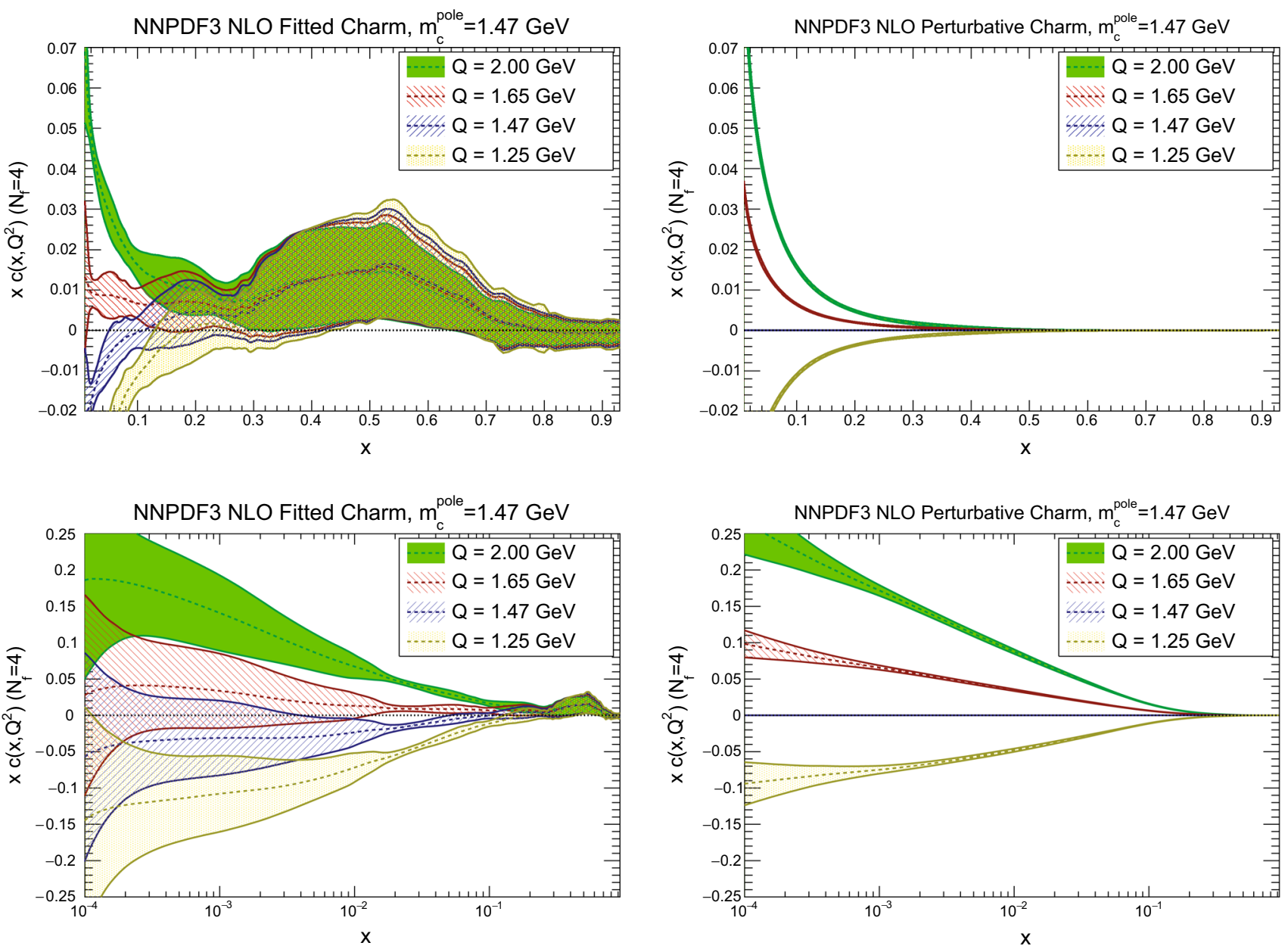

Fig. 14 The charm PDF (when $m_{c}=1.47 \mathrm{GeV}$ ) plotted as a function $x$ on a linear (top) or logarithmic scale (bottom) for four low scale values $Q=1.25,1.47,1.65$ and $2 \mathrm{GeV}$ in the four-flavour scheme. Both fitted

(left) and perturbative (right) charm are shown. Note that in a matched scheme the charm PDF would become scale independent for $Q<m_{c}$
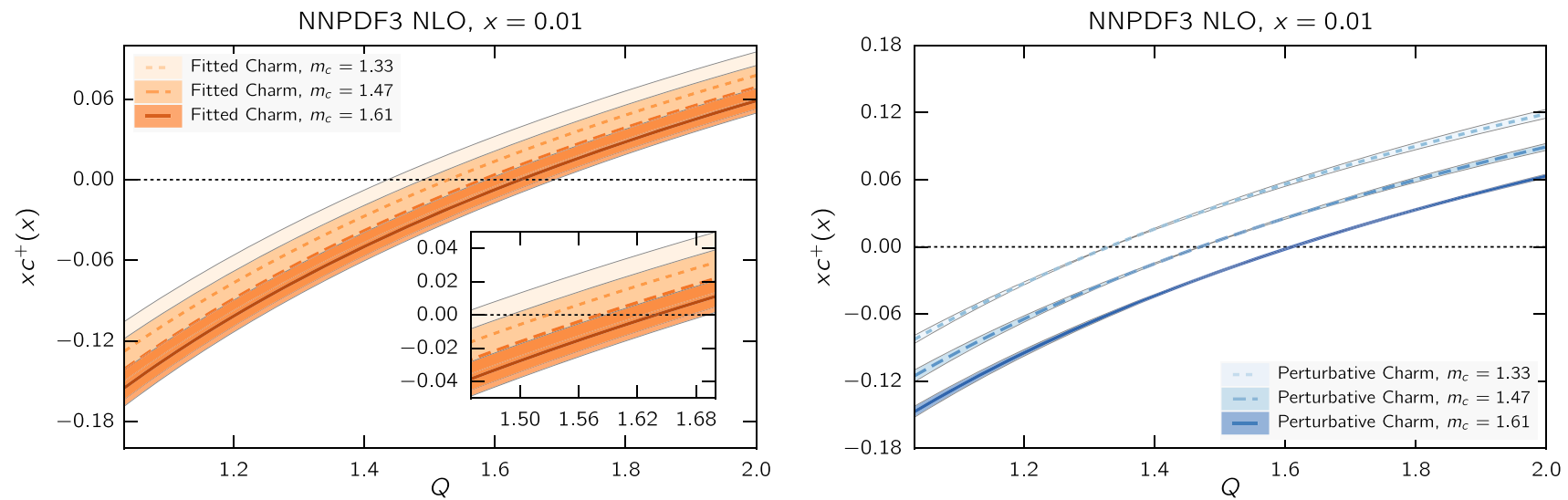

Fig. 15 The charm PDF in the four-flavour scheme as a function of scale at $x=0.01$ for different values of the heavy quark mass with fitted (left) and perturbative (right) charm

for intrinsic charm at about the one-sigma level. The intrinsic charm contribution to the momentum fraction, when the EMC data are included, is then around $0.5 \pm 0.3 \%$, entirely consistent with a power suppression of order $\Lambda^{2} / m_{c}^{2}$. Without the EMC data, the fraction increases to $1.4 \pm 1.2 \%$ (taking into account the perturbative contribution at this scale), 

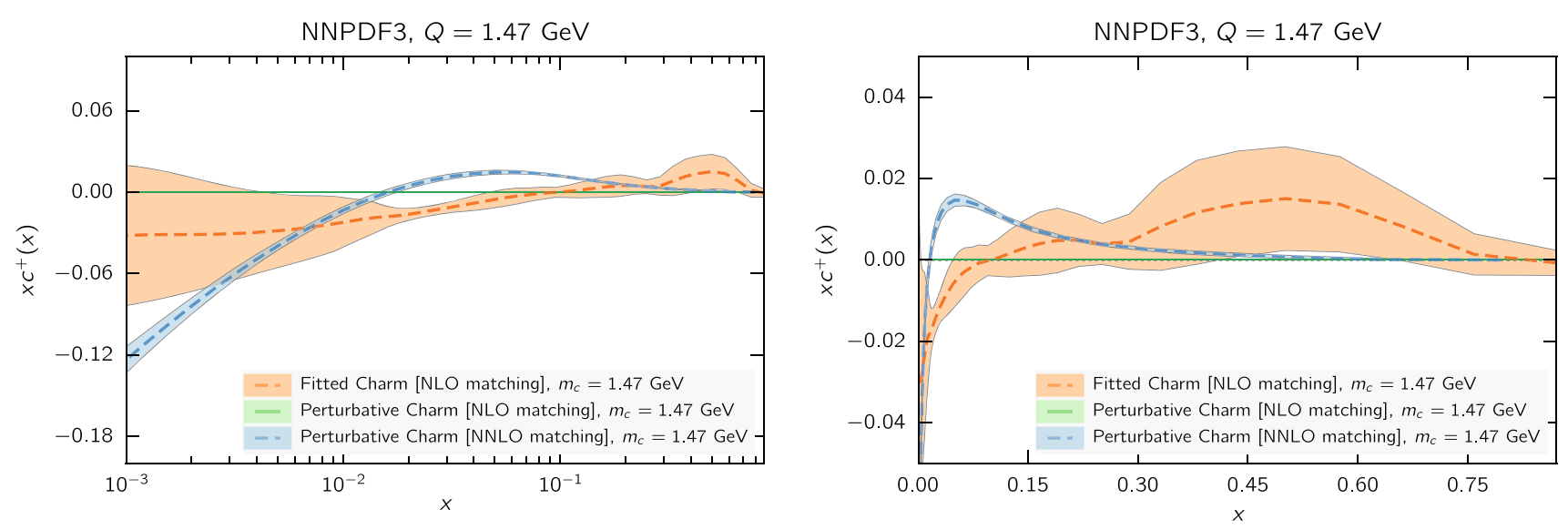

Fig. 16 The charm PDF plotted vs. $x$ on a logarithmic (left) or linear (right) scale, when $Q=m_{c}=1.47 \mathrm{GeV}$. The fitted and perturbative NLO and NNLO (see text) results are compared

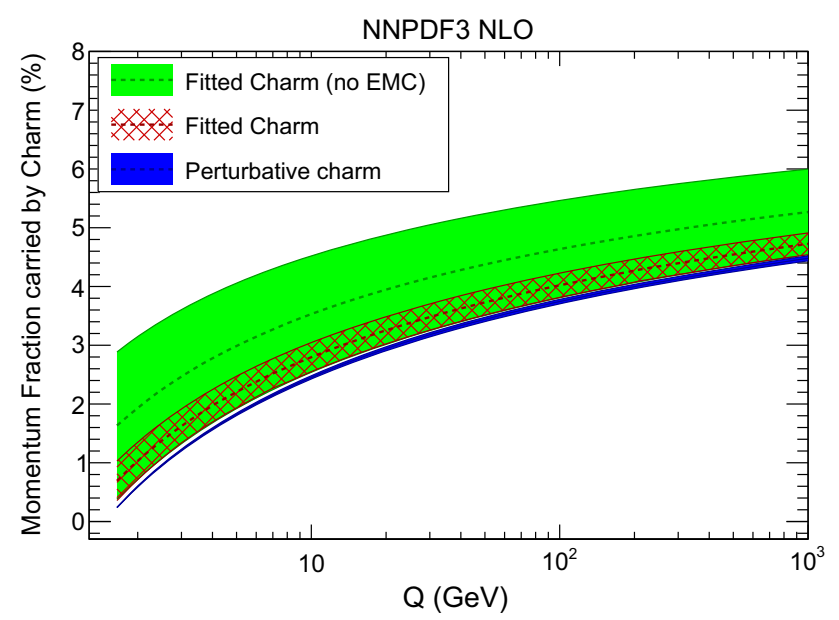

Fig. 17 The charm momentum fraction $C\left(Q^{2}\right)$, Eq. (5), as a function of scale with perturbative and with fitted charm, with and without the EMC data included in the fit

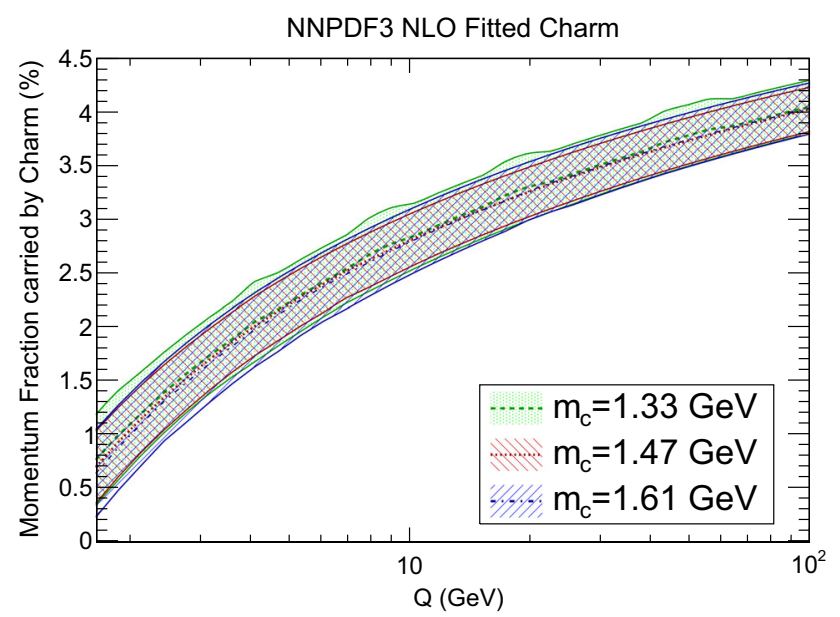

Table 3 The charm momentum fraction $C\left(Q^{2}\right)$ at a low scale $Q=$ $1.65 \mathrm{GeV}$ with perturbative charm, and with fitted charm with and without the EMC data included. The momentum fractions for several CT14IC PDF sets are also given for comparison (see text)

\begin{tabular}{ll}
\hline PDF set & $C(Q=1.65 \mathrm{GeV})$ \\
\hline NNPDF3 perturbative charm & $(0.239 \pm 0.003) \%$ \\
NNPDF3 fitted charm & $(0.7 \pm 0.3) \%$ \\
NNPDF3 fitted charm (no EMC) & $(1.6 \pm 1.2) \%$ \\
CT14IC BHPS1 & $1.3 \%$ \\
CT14IC BHPS2 & $2.6 \%$ \\
CT14IC SEA1 & $1.3 \%$ \\
CT14IC SEA2 & $2.2 \%$ \\
\hline
\end{tabular}

though the allowed range for $C(Q)$ is reduced once the EMC data are included.

At high scale, as shown in Fig. 17, the momentum fraction carried by the charm PDF is dominated by its perturba-

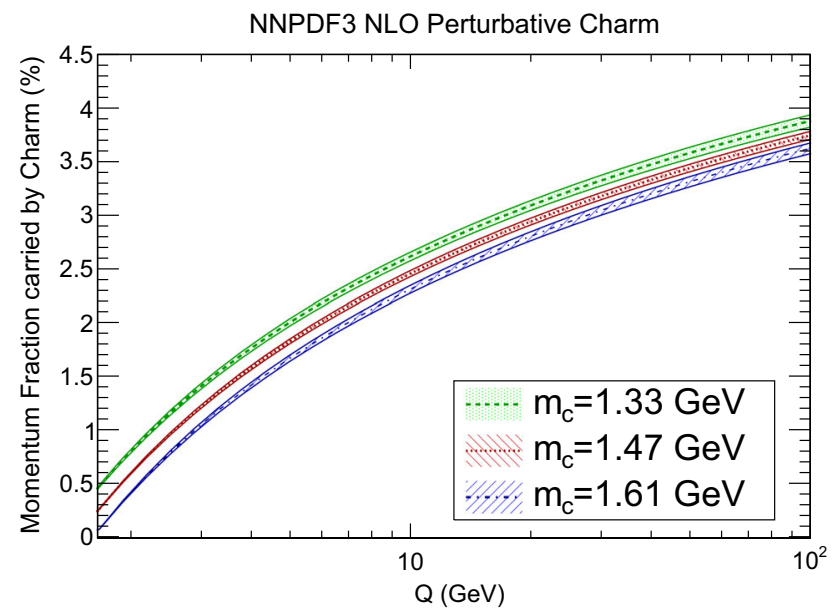

Fig. 18 Same as Fig. 17, with three different values of the pole charm mass, for fitted (left) and perturbative (right) charm 

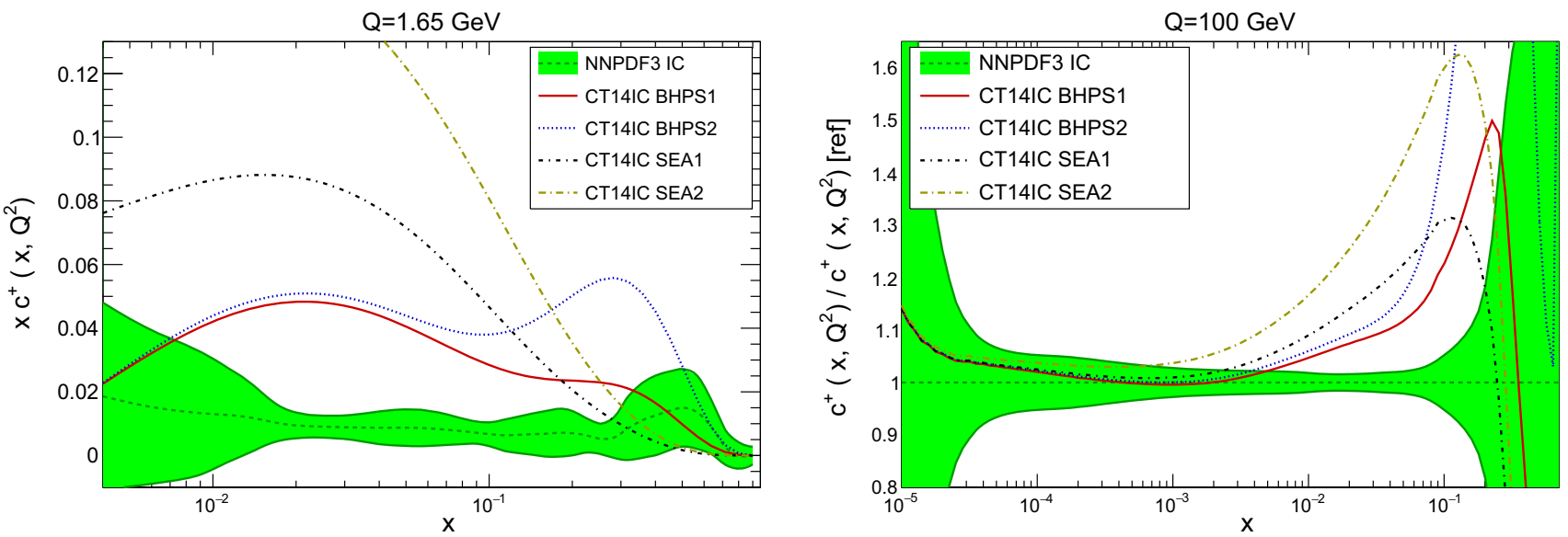

Fig. 19 Comparison of the NNPDF3 fitted charm PDF with the different CT14IC models of $[2,15]$ at a low scale $Q=1.65 \mathrm{GeV}$ (left) and at a high scale $Q=100 \mathrm{GeV}$ (right)

tive component, and it becomes about $5 \%$ at $Q=1 \mathrm{TeV}$. However, it is clear from Fig. 18 that the momentum fraction of fitted charm is essentially independent of the charm mass at all scales, and is thus determined exclusively by the data. On the other hand, with perturbative charm the momentum fractions obtained for different values of the mass do not overlap at the one-sigma level, even at high scale, and they are thus instead determined by the assumed value of the mass.

In order to further understand the features of our fitted intrinsic component we compare it to previous determinations based on models. To this purpose, we compare our fitted charm with the charm PDFs recently given in Refs. [2,15] within the framework of the CT14 NNLO PDF determination. In this analysis two different models for intrinsic charm were considered: a BHPS scenario [98] in which charm at $Q_{0}=1.3 \mathrm{GeV}$ has a valence-like shape,

$c\left(x, Q_{0}\right)=A x^{2}\left[6 x(1+x) \ln x+(1-x)\left(1+10 x+x^{2}\right)\right]$,

which peaks around $x \sim 0.25$, and a SEA model in which charm is assumed to have the same shape as the light quark sea:

$c\left(x, Q_{0}\right)=A\left[\bar{d}\left(x, Q_{0}\right)+\bar{u}\left(x, Q_{0}\right)\right]$.

In both cases, the only free parameter of the model is the positive-definite normalization $A$, for which two different values, corresponding to two different momentum fractions, are considered (see Table 3 ).

In Fig. 19 we compare the NNPDF3 fitted charm PDF with the four CT14 IC models both at a low scale $Q=1.65 \mathrm{GeV}$ and a high scale $Q=100 \mathrm{GeV}$. While the fitted charm is qualitatively similar to the BHPS model [98], it is entirely different from the SEA model. At small $x$ the NNPDF3 fitted charm is smaller than all the models, and it peaks at larger values of $x$ than the BHPS model. At high scale, there is good agreement between our fitted charm and the models in the region where perturbative evolution dominates, $x \lesssim 10^{-3}$, with more substantial differences at medium and large $x$ : for example, for $x \simeq 0.2$ the charm PDF in the BHPS 1 model is $40 \%$ larger than in our fit. Comparing the momentum fractions in Table 3, our fitted charm result with EMC data prefers a rather lower momentum fraction than was considered in Refs. [2,15]. In fact it seems that the BHPS model, with normalization reduced by $40 \%$ or so from that used in BHPS1, might be in reasonable agreement with our fit at large $x$.

We also find that results contradict the claim from the authors of Ref. [14] (based on the JR PDF fit framework) that values of the charm momentum fraction of $C(Q)$ at the $0.5 \%$ level are excluded at the four-sigma level. Note, however, that none of these models reproduce the features of our best-fit charm at small $x$, and specifically the undershoot in comparison to the perturbative behaviour discussed above.

Our general conclusion is thus that if the EMC data are reliable, then charm is compatible with perturbative behaviour at small $x \lesssim 0.1$, where it vanishes at a scale which at NLO turns out to be $Q_{0} \sim 1.6 \mathrm{GeV}$, while it has an intrinsic component at large $x$ which carries about a percent of the proton momentum at low scale. Not including a fitted charm component with $m_{c}=1.47 \mathrm{GeV}$ would thus bias the PDF determination both at small and large $x$, with the large $x$ bias localized at low scale and the small $x$ bias also affecting high-scale physics. The small $x$ bias would, however, mostly disappear if PDFs were provided with uncertainties related to missing higher-order corrections and the value of the charm mass, or if the mass value was raised. 

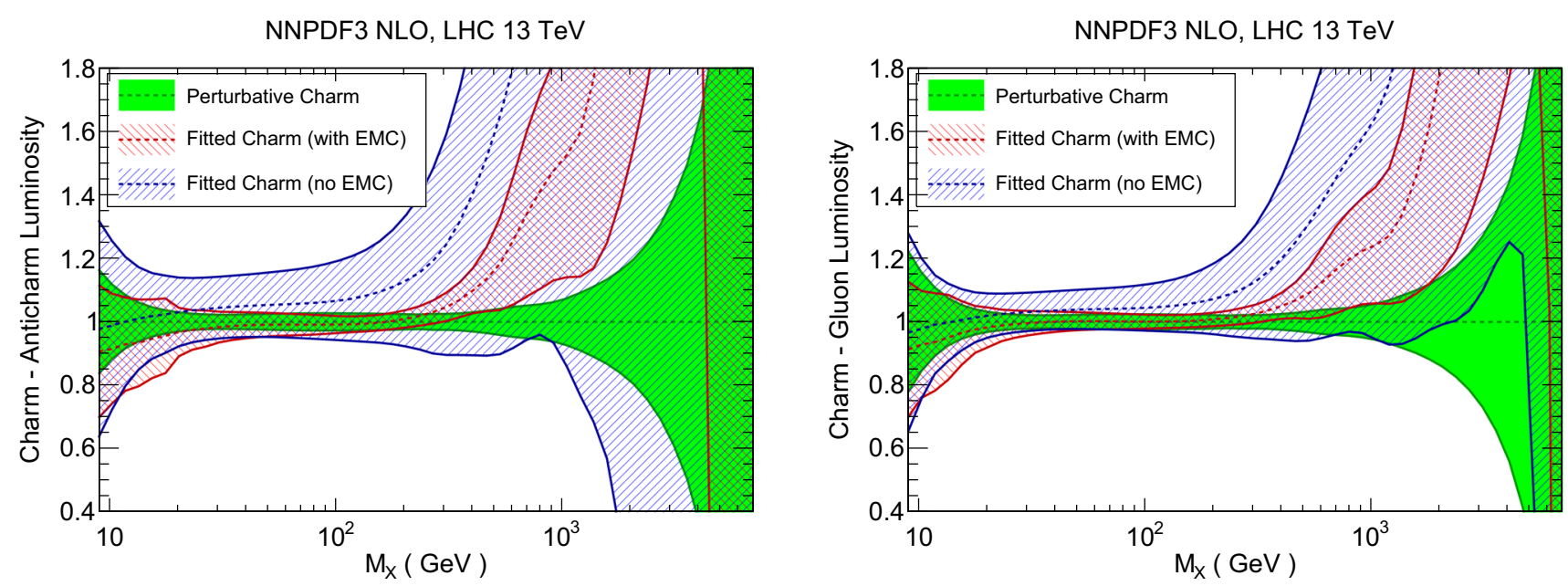

Fig. 20 Parton luminosities at the LHC $13 \mathrm{TeV}$ as a function of the invariant mass $M_{X}$ of the final state, computed using the PDF sets with perturbative charm, and with fitted charm with and without EMC data. The charm-anticharm (left) and charm-gluon luminosities (right) are shown

\section{LHC phenomenology}

We now discuss the implications of fitting charm for LHC phenomenology. First, we compare parton luminosities computed with fitted or perturbative charm, and specifically show at the level of luminosities the improved stability upon variation of the charm mass that was already discussed in Sect. 3.2 at the level of PDFs. We then turn to specific processes: first, we discuss the effect of fitting charm on standard candles, thereby showing that fitting charm is advantageous for more robust uncertainty estimation. Then we consider representative LHC processes which are sensitive to charm and could be used for a more accurate charm PDF determination: charm quark pair production and $Z$ production in association with charm quarks.

\subsection{Parton luminosities}

In Fig. 20 we compare parton luminosities (defined as in Ref. [99]) involving charm at the LHC $13 \mathrm{TeV}$, plotted as a function of the invariant mass $M_{X}$ of the final state, for the PDF sets with perturbative and fitted charm, with and without EMC data, discussed in Sect. 3. We show the charmanticharm and charm-gluon luminosities, which are relevant for charm-dominated processes at the LHC, such as $D$ meson production at large $p_{T}$ and rapidity, where the $c \bar{c}$ process becomes important, or $\gamma / Z+D$ production, which at the Born level is driven by the $g c$ luminosity. We find that when the EMC data are included, the uncertainty in the luminosity with fitted charm is similar to that when charm is perturbative for scales $M_{X} \sim 100 \mathrm{GeV}$, and larger than it by a factor 3 or 4 for higher or lower scale, while if EMC data are not included the uncertainty with fitted charm is substantially larger for all scales. This suggests that the determination of the lumi- nosities with purely perturbative charm might be unreliable, with underestimated uncertainty and possibly a biased central value, particularly at high invariant masses. In Fig. 21 we show luminosities involving light quarks and gluons. In this case, the uncertainties are similar with fitted or perturbative charm provided EMC data are included.

The difference between fitted and perturbative charm is particularly apparent in the dependence of luminosities on the value of the charm mass, which is shown in Fig. 22 (for the light quark-antiquark and the gluon-gluon luminosity). A marked increase in stability is seen in the $q \bar{q}$ luminosity for all $M_{X}$ when charm is fitted. This means that if charm is not fitted, the choice of charm mass is a possible source of bias. The reduced dependence on the value of $m_{c}$ becomes especially striking for luminosities involving charm: as shown in Fig. 23, the spread in central values for the charm-anticharm luminosity as the charm mass is varied is about $15 \%$ for perturbative charm and about $2 \%$ for fitted charm for all $20 \mathrm{GeV}<M_{X}<1 \mathrm{TeV}$. Similar conclusions hold for the cg luminosity.

\subsection{LHC standard candles}

We now study the impact of the fitted charm PDFs for the calculation of standard candles at the LHC. We start with total cross sections and then consider some differential distributions, all at the LHC $13 \mathrm{TeV}$.

\subsubsection{Total cross sections}

We first consider Higgs and top production. We have computed the total inclusive Higgs production cross section in the gluon fusion channel using the ggHiggs code v3.2 [100] to NLO, including full dependence on the top, bottom and 

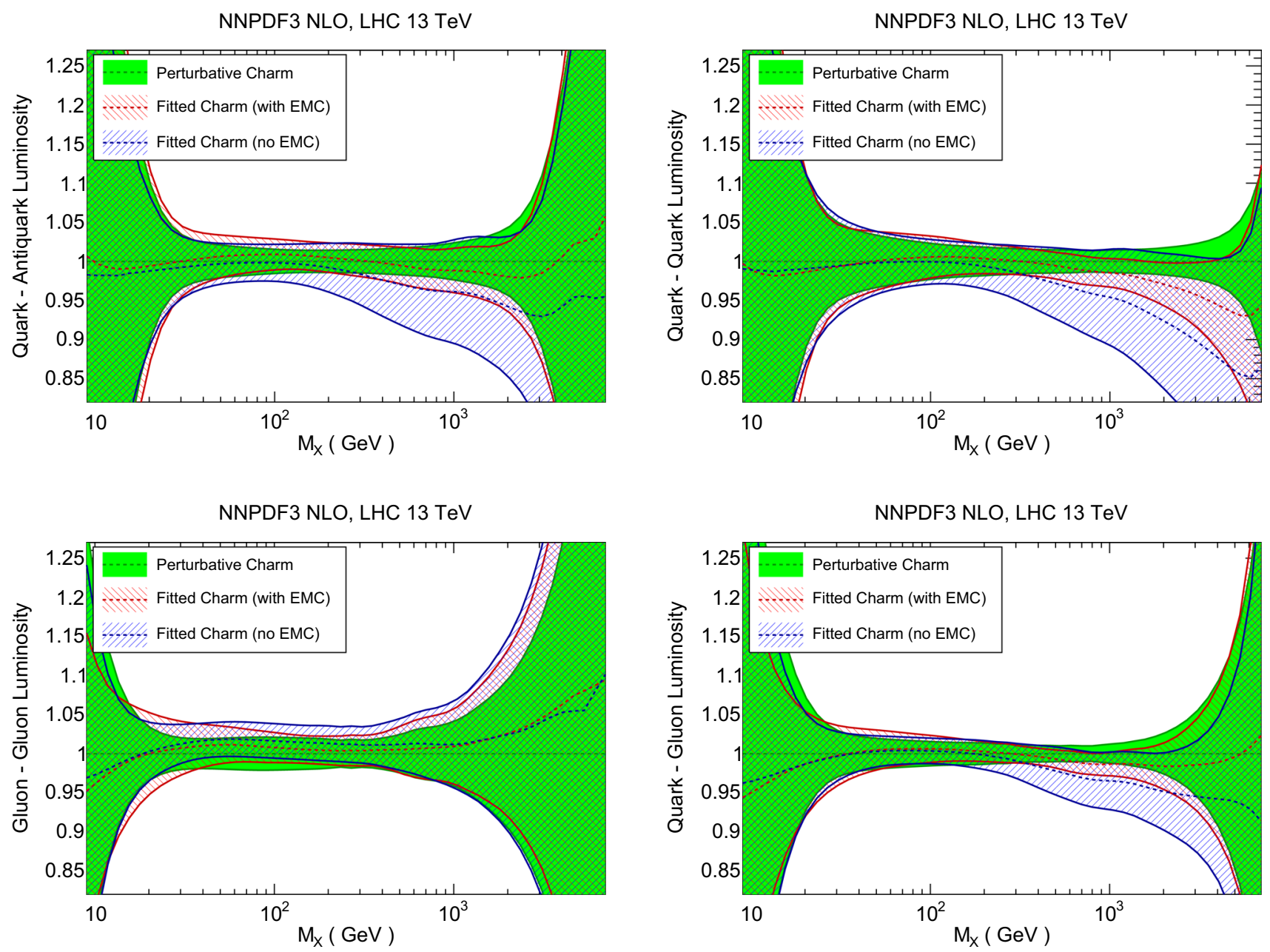

Fig. 21 Same as Fig. 20, but for quark-antiquark (top left), quark-quark (top right), gluon-gluon (bottom left) and quark-gluon (bottom right) luminosities

charm masses, for $\mu_{F}=\mu_{R}=m_{H} / 2$ and $m_{H}=125 \mathrm{GeV}$. We have also computed the inclusive top quark pair production cross section at NLO using top ++ v2.0 [101]. Results are collected in Table 4 and represented in Fig. 24. Note that the uncertainty shown is the PDF uncertainty only (not including $\alpha_{s}$ variation). In both cases, the impact of fitting charm on the cross section is moderate, both for central values and uncertainties, and while the cross section is almost independent of the charm mass for perturbative charm, it varies a little more when the charm is fitted. The overall uncertainty is thus a little larger with fitted charm, reflecting the slightly increased uncertainty in the gluon-gluon luminosity.

Next, we have computed the total cross section for $W$ and $Z$ production at NLO at the LHC 13 TeV using MCFM [102]. We include the leptonic decays of the gauge bosons, and we impose standard acceptance requirements for the final-state leptons, namely $p_{T}^{l} \geq 20 \mathrm{GeV}$ and $\left|\eta_{l}\right| \leq 2.5$. Results are presented in Fig. 25 and collected in Table 4. Here, while again results with perturbative or fitted charm are very simi- lar, an improvement in stability with respect to the choice of $m_{c}$ when charm is fitted is clearly visible for $Z$ production. Also, we see that whether or not we include the EMC data makes very little difference to these standard candles.

As a general conclusion, we find that the variation of total cross section for LHC standard candles as the charm mass is varied in a very conservative range is a small fraction of the PDF uncertainty. This conclusion is in agreement with previous studies of the dependence of global fit results on the charm mass (but with perturbative charm only) presented in Refs. [80, 103-105].

\subsubsection{Differential distributions}

We now turn to differential distributions for Higgs production in gluon fusion, top-pair production and $W, Z$ electroweak gauge-boson production at $13 \mathrm{TeV}$. All calculations have been performed at NLO using MadGraph5_aMC@NLO [106] 

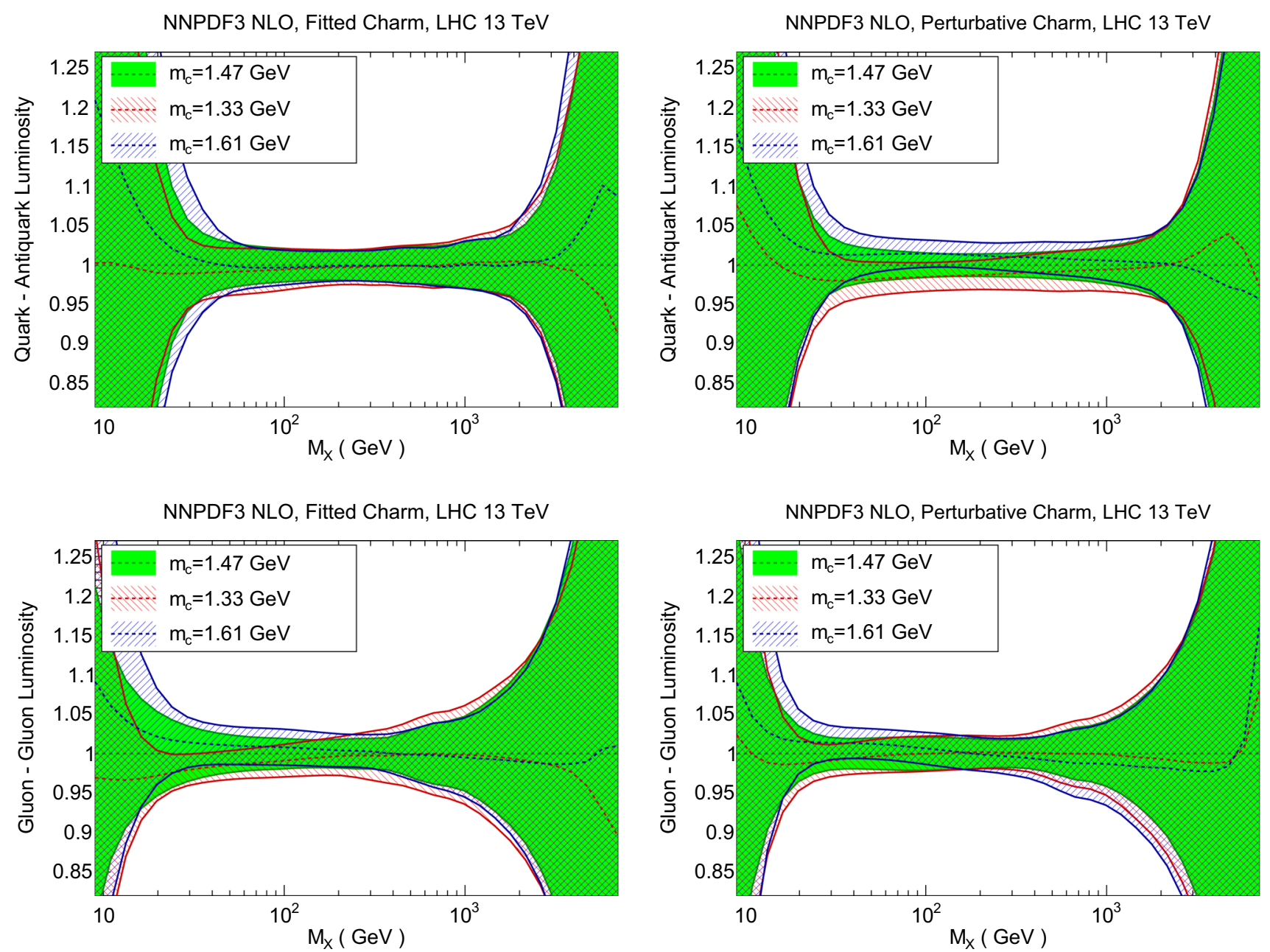

Fig. 22 Same as Fig. 21, but now comparing the quark-antiquark (top) and gluon-gluon (bottom) luminosities for different values of the pole charm mass, for fitted (left) and perturbative charm (right)

interfaced to aMCfast [107] and APPLgrid [71]. The choice of binning, kinematical cuts and final-state decays in these processes are the same as those used in the SM-PDF study [108], to which we refer for further information. In each case, we compare results obtained with perturbative charm, and with fitted charm when EMC data are included or not. All uncertainties shown are PDF uncertainties only. In addition, we also compare results for fitted charm (with and without EMC data) obtained with different values of the charm mass, and the corresponding results in the case of fits with perturbative charm.

In Fig. 26 we show the Higgs transverse momentum and rapidity, the transverse momentum of the $Z$ boson, the rapidity of the $W$ boson, and the invariant mass and top quark rapidity in $t \bar{t}$ production. In all cases, we observe considerable stability of central values when moving from perturbative to fitted charm, with only a small increase in uncertainty for fitted charm, and no significant difference found when EMC data are excluded.
Then in Figs. 27 and 28 we show the comparison of the differential distributions of Fig. 26 upon variations of the charm quark mass, both for fitted and perturbative charm PDFs. For the gluon-initiated processes ( $g g H$ and $t \bar{t}$ ) the results with fitted and perturbative charm are quite similar: the main effect of fitted charm is to give a more conservative estimate of the overall uncertainty. For quark-induced processes ( $W$ and $Z$ ) we see a marked improvement in the stability upon charm mass variations for fitted charm, particularly at low $p_{T}$ and at central rapidities: this is a direct reflection of the reduced sensitivity to charm mass variations in the medium $x$ region when charm is fitted.

We conclude that for LHC observables which do not depend directly on the charm PDF, both at the inclusive and differential level, the impact of fitting charm is moderate: for gluon dominated processes it provides a more conservative error estimate, while for quark-induced processes it offers a reduction in the (already quite weak) dependence on the value of $m_{c}$. 

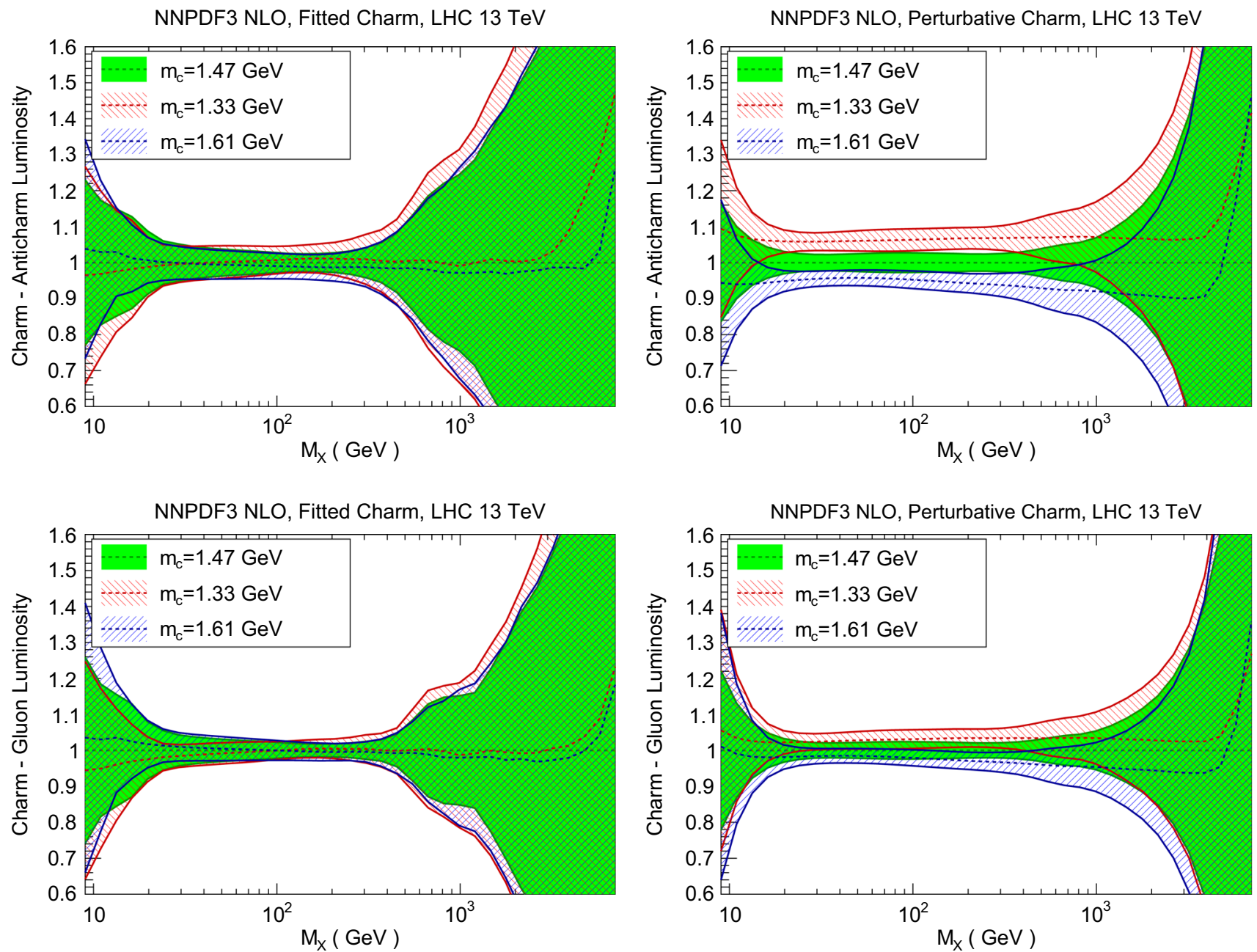

Fig. 23 Same as Fig. 22 for charm-anticharm (top) and charm-gluon luminosities (bottom)

Table 4 Numerical values for the cross sections represented in Figs. 24 and 25

\begin{tabular}{lllll}
\hline Process & Charm PDF & $m_{c}=1.33 \mathrm{GeV}$ & $m_{c}=1.47 \mathrm{GeV}$ & $m_{c}=1.61 \mathrm{GeV}$ \\
\hline$\sigma(g g \rightarrow h)[\mathrm{pb}]$ & Fitted & $35.5 \pm 0.7$ & $35.7 \pm 0.5$ & $35.8 \pm 0.7$ \\
& Fitted (no EMC) & - & $36.0 \pm 0.7$ & - \\
& Perturbative & $35.5 \pm 0.7$ & $35.4 \pm 0.6$ & $35.5 \pm 0.6$ \\
$\sigma(t \bar{t})[\mathrm{pb}]$ & Fitted & $733 \pm 26$ & $734 \pm 18$ & $734 \pm 20$ \\
& Fitted (no EMC) & - & $738 \pm 20$ & - \\
& Perturbative & $731 \pm 20$ & $731 \pm 15$ & $726 \pm 21$ \\
$\sigma\left(W^{+} \rightarrow l^{+} v\right)[\mathrm{nb}]$ & Fitted & $6.09 \pm 0.14$ & $6.14 \pm 0.13$ & $6.04 \pm 0.13$ \\
& Fitted (no EMC) & - & $6.15 \pm 0.12$ & - \\
& Perturbative & $5.97 \pm 0.10$ & $6.03 \pm 0.10$ & $6.11 \pm 0.10$ \\
$\sigma\left(W^{-} \rightarrow l^{-} v\right)[\mathrm{nb}]$ & Fitted & $4.42 \pm 0.10$ & $4.43 \pm 0.09$ & $4.40 \pm 0.09$ \\
& Fitted (no EMC) & - & $4.44 \pm 0.08$ & - \\
& Perturbative & $4.38 \pm 0.07$ & $4.41 \pm 0.07$ & $4.47 \pm 0.07$ \\
$\sigma\left(Z \rightarrow l^{+} l^{-}\right)[\mathrm{nb}]$ & Fitted & $1.412 \pm 0.028$ & $1.410 \pm 0.026$ & $1.410 \pm 0.025$ \\
& Fitted (no EMC) & - & $1.400 \pm 0.023$ & - \\
& Perturbative & $1.376 \pm 0.022$ & $1.380 \pm 0.021$ & $1.5403 \pm 0.021$ \\
\hline
\end{tabular}


Inclusive gluon-fusion Higgs production @ NLO, LHC $13 \mathrm{TeV}$

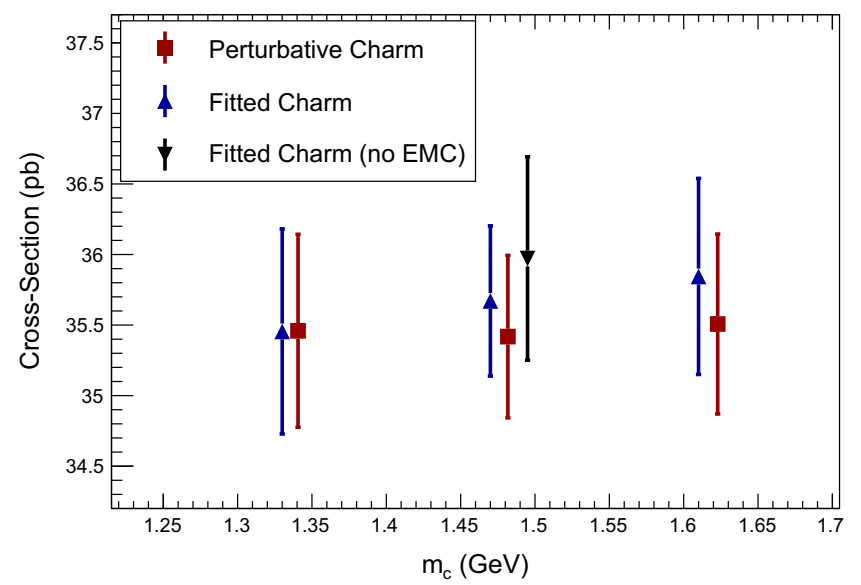

Fig. 24 The NLO cross sections for Higgs production in gluon fusion (left) and inclusive top quark pair production (right) at the LHC $13 \mathrm{TeV}$ with fitted or perturbative charm and $m_{c}^{\text {pole }}=1.33,1.47$ and 1.61
Top quark pair production @ NLO, LHC 13 TeV

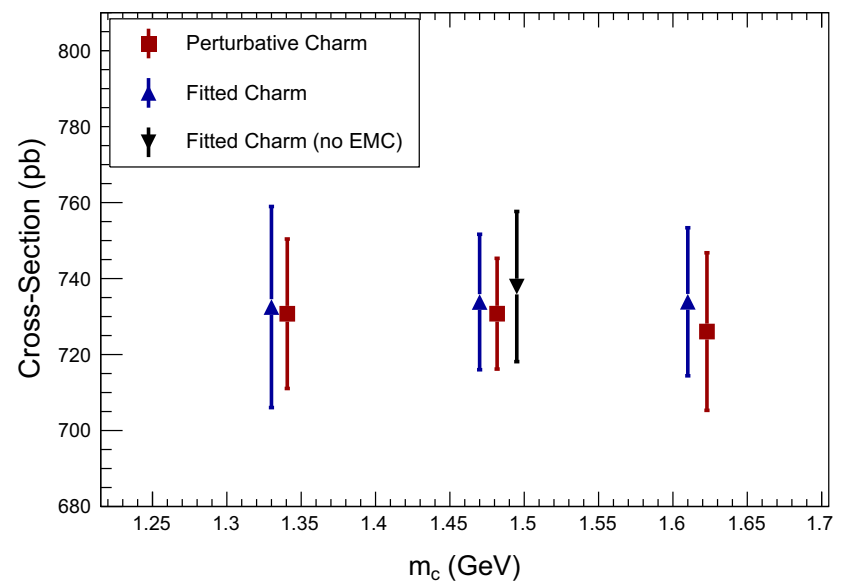

$\mathrm{GeV}$. We also show the result with fitted charm and no EMC data for $m_{c}^{\text {pole }}=1.47 \mathrm{GeV}$. The uncertainty shown is the PDF uncertainty only (not including i.e. $\alpha_{s}$ variations)
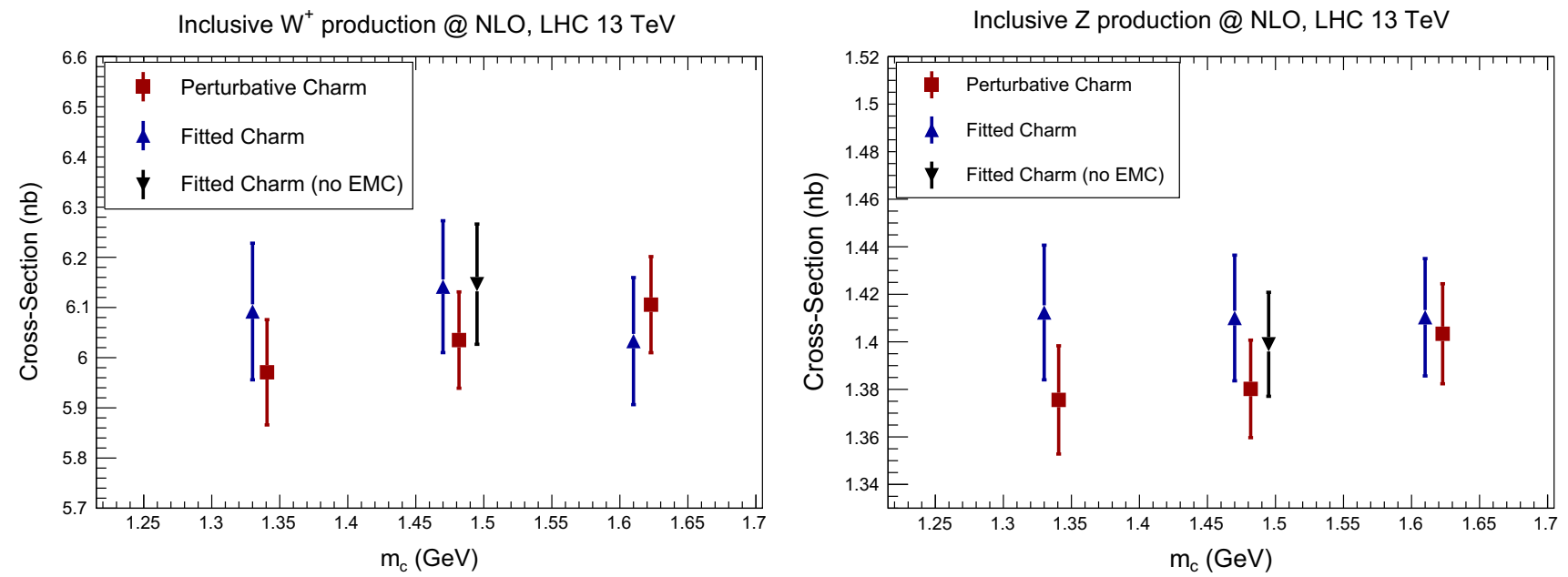

Fig. 25 Same as Fig. 24 for the cross section for the inclusive production of $W^{+}$(left) and $Z$ (right) bosons at the LHC 13 TeV, including leptonic branching fractions and standard acceptance cuts

\subsection{Probing charm at the $\mathrm{LHC}$}

We now turn to LHC observables which do depend directly on the charm PDF, and which could thus be used for its determination. Such observables include prompt photon production in association with $D$ mesons [109-111], $Z$ boson production together with charm quarks [112-114] and open $D$ meson production [115-119], as well as more exotic processes such as double charmonium production [21], and inclusive and diffractive Higgs production $[120,121]$. Here we concentrate on two illustrative cases, namely $Z+$ charm and $c \bar{c}$ production. We will specifically discuss the kinematic regions which are sensitive to the charm PDF at large $x$, and which could therefore be used to confirm our first evidence, discussed in
Sect. 3.4, for an 'intrinsic' component of charm: as we will see, these are the regions of large $p_{T}$ or large rapidity.

\subsubsection{Z production in association with charm quarks}

In Fig. 29 we show representative leading-order Feynman diagrams for the production of a $Z$ boson in association with a charm quark at hadron colliders, driven by the $c g$ luminosity. The calculation of this process at NLO has been performed with MCFM interfaced to APPLgrid, and cross-checked with MadGraph5_aMC@NLO interfaced to aMCfast. It is beyond the scope of this paper to perform a complete feasibility study of this measurement, so we neglect the hadronization of the charm quark into a $D$ meson, which 


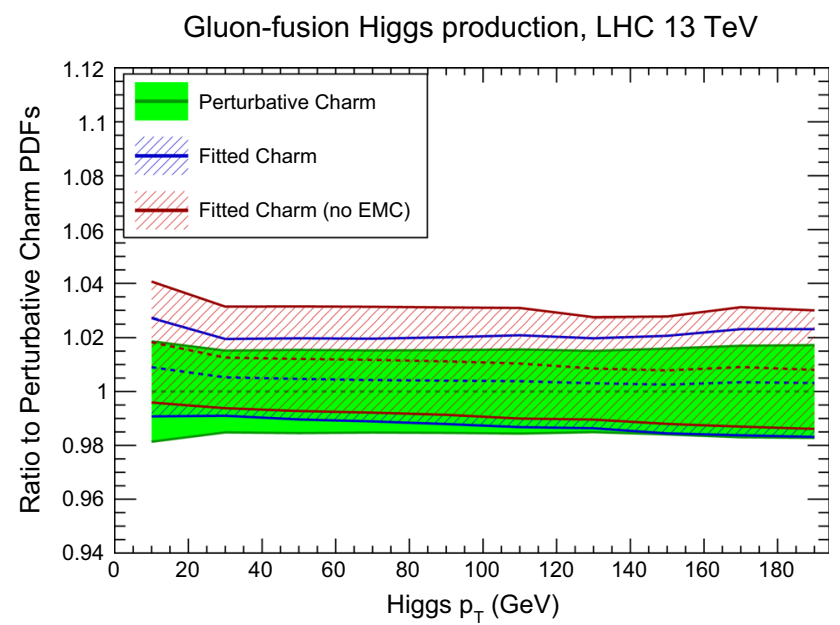

Inclusive $\mathrm{Z}$ production, LHC $13 \mathrm{TeV}$

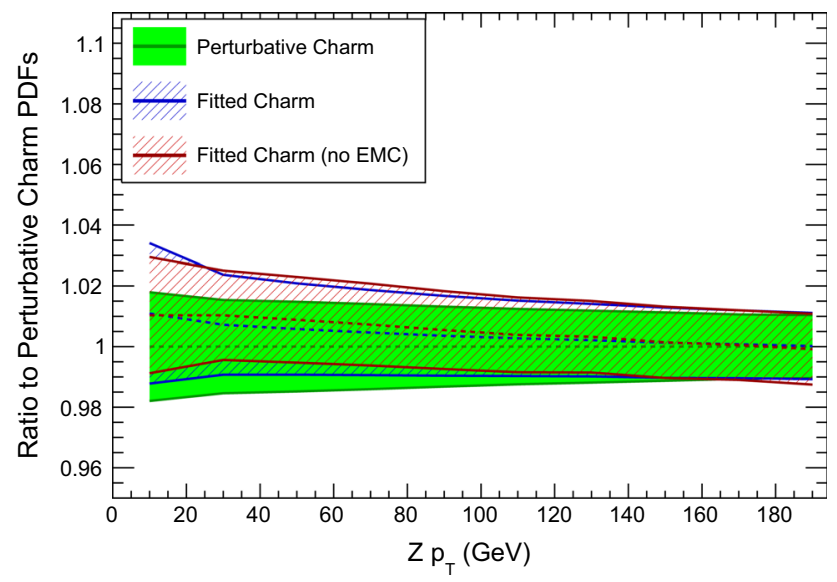

Top quark pair production, LHC $13 \mathrm{TeV}$

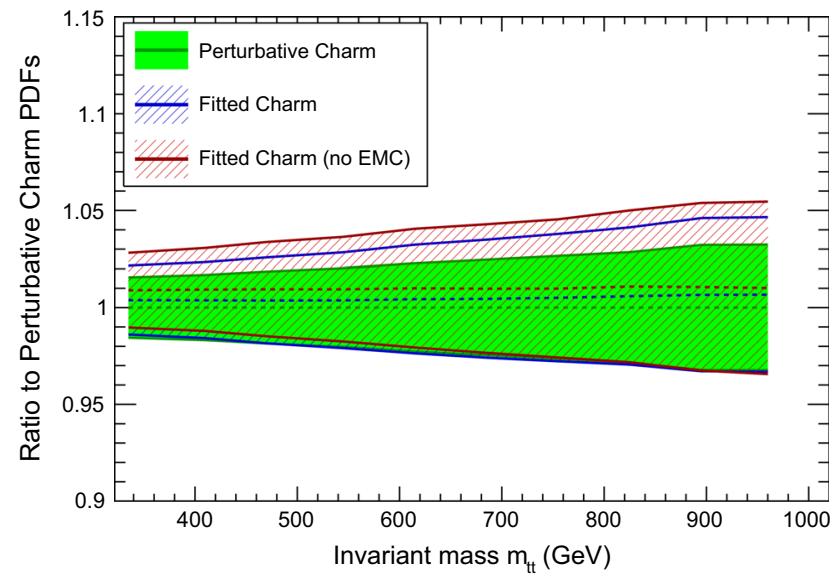

Fig. 26 Comparison of the results of the baseline fit with perturbative charm with the corresponding fitted charm PDFs, with and without the EMC data included for NLO differential distributions at $13 \mathrm{TeV}$.

does not significantly affect the sensitivity of this process to the charm PDF. In Fig. 30 we show the rapidity distribution and the transverse momentum of the $Z$ boson in $Z+c$

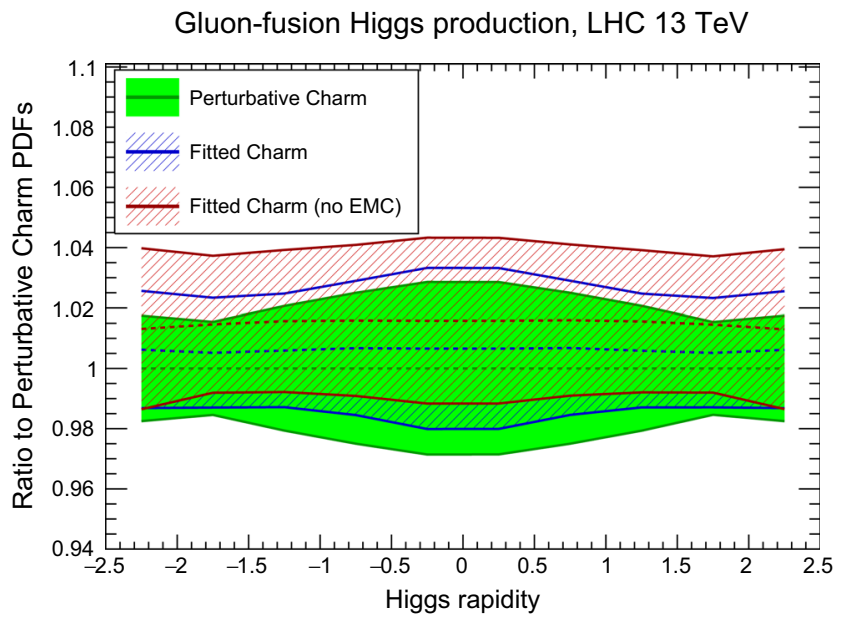

Inclusive W production, LHC $13 \mathrm{TeV}$

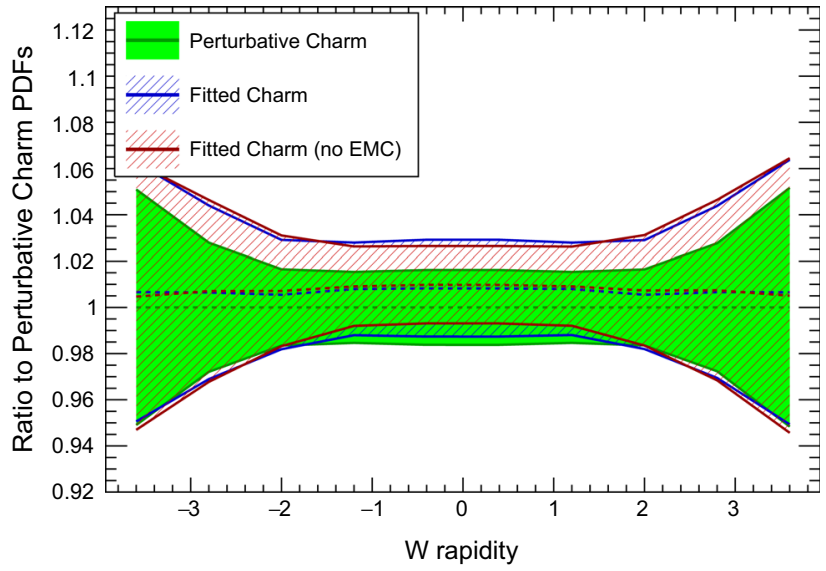

Top quark pair production, LHC $13 \mathrm{TeV}$

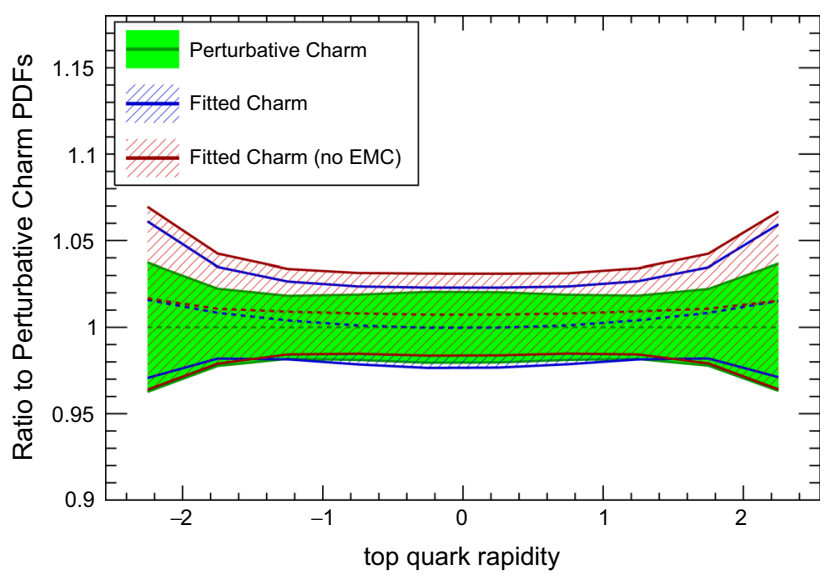

From top to bottom and from left to right we show the Higgs transverse momentum and rapidity, the $p_{T}$ of the $Z$ boson, the rapidity of the $W$ boson, and $m_{t \bar{t}}$ and $y_{t}$ in $t \bar{t}$ production

production at the LHC $13 \mathrm{TeV}$. We compare the results of perturbative or fitted charm PDFs, in the latter cases with and without the EMC data included. We also show predic- 


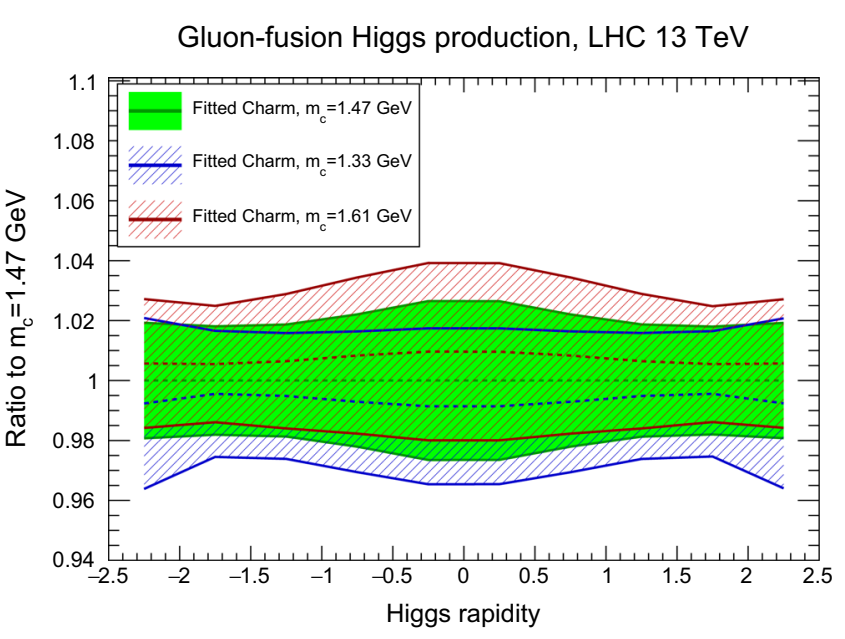

Top quark pair production, LHC $13 \mathrm{TeV}$

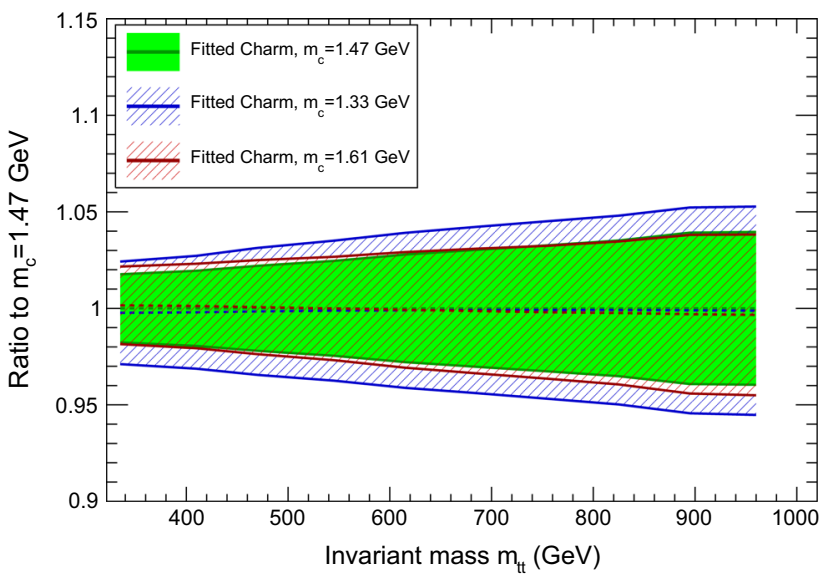

Gluon-fusion Higgs production, LHC $13 \mathrm{TeV}$

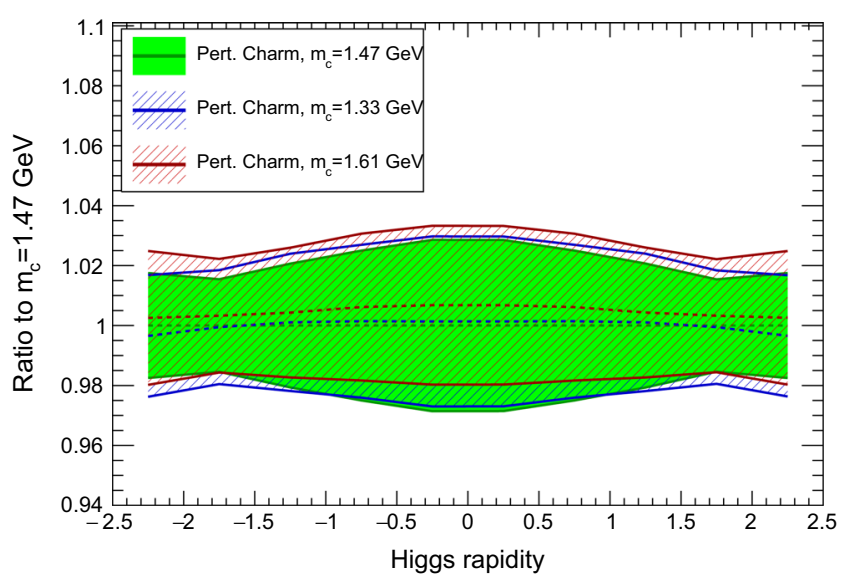

Top quark pair production, LHC $13 \mathrm{TeV}$

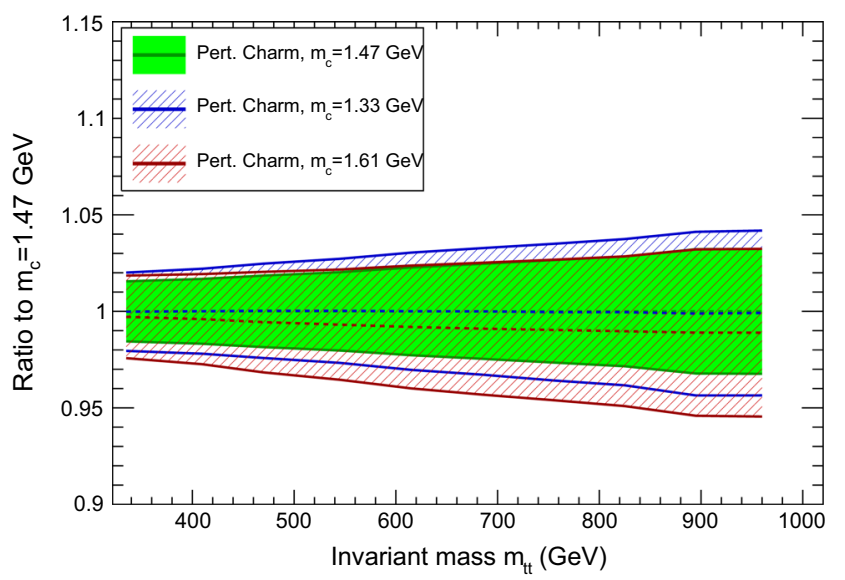

Fig. 27 The Higgs rapidity distribution and the invariant mass distribution of top quark pairs in $t \bar{t}$ production, as in Fig. 26, but now comparing different values of the charm mass with fitted charm (left) and perturbative charm (right)

tions obtained using the four CT14NNLO sets discussed in Sect. 3.4.

In the case of the $Z$ rapidity distribution percentage differences in central values are moderate at central rapidity but increase substantially in the forward region. In particular, in the LHCb acceptance region, $2.0 \leq y_{Z} \leq 4.5$, an enhancement of the cross section by a factor 2 or more is possible in the case of fitted charm, compared to the baseline result with perturbative charm (for a recent study of charm PDF constraints in $Z+c$ production at $\mathrm{LHCb}$ see e.g. Ref. [112]). However in this region PDF uncertainties in the fitted charm case are large, and the three NNPDF sets shown agree with each other at the one-sigma level in the entire range of $y_{Z}$. This means that more accurate data for this observable could provide a useful constraint on the charm PDF.

In the case of the transverse momentum distribution of $Z$ bosons, the NNPDF sets with fitted charm and the CT14 sets based on the BPHS model exhibit a substan- tial enhancement of the cross section at large $p_{T}^{Z}$ in comparison to the perturbative charm baseline. For the fitted charm NNPDF3 PDFs with EMC data, this enhancement could be as large as a factor 2 (at the one-sigma level) for $p_{T}^{Z} \simeq 700 \mathrm{GeV}$. Once again, however, results obtained with perturbative and fitted charm PDFs are consistent with each other within the large uncertainties, so also in this case more accurate measurements could provide a useful constraint.

Turning things around, an accurate measurement at high rapidity and transverse momentum could rule out perturbative charm. Also, in the central rapidity region, an accurate enough measurement could confirm the undershoot in the fitted charm case which is seen in Fig. 30, and, though smaller in absolute terms, it is as significant as the large rapidity excess on the scale of present-day uncertainties. A full NNLO analysis will be required in order to arrive at a definite conclusion, especially in view of the fact that, as discussed in Sect. 3.4, the fitted charm might be reabsorbing higher-order corrections. 
Inclusive $\mathrm{Z}$ production, LHC $13 \mathrm{TeV}$

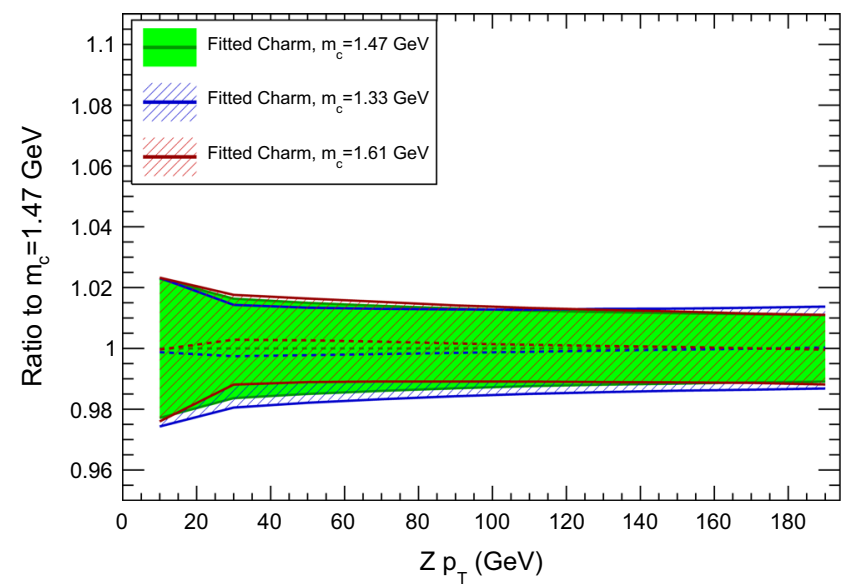

Inclusive W production, LHC $13 \mathrm{TeV}$

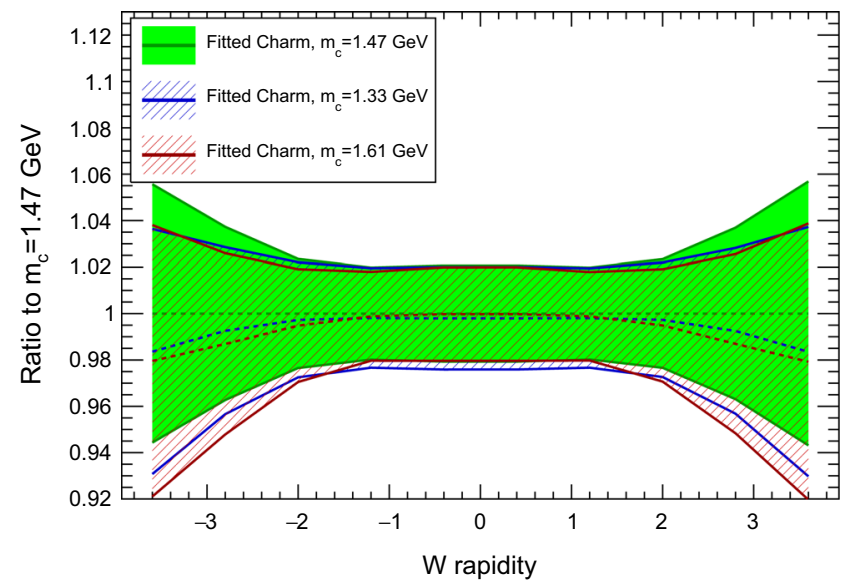

Inclusive Z production, LHC $13 \mathrm{TeV}$

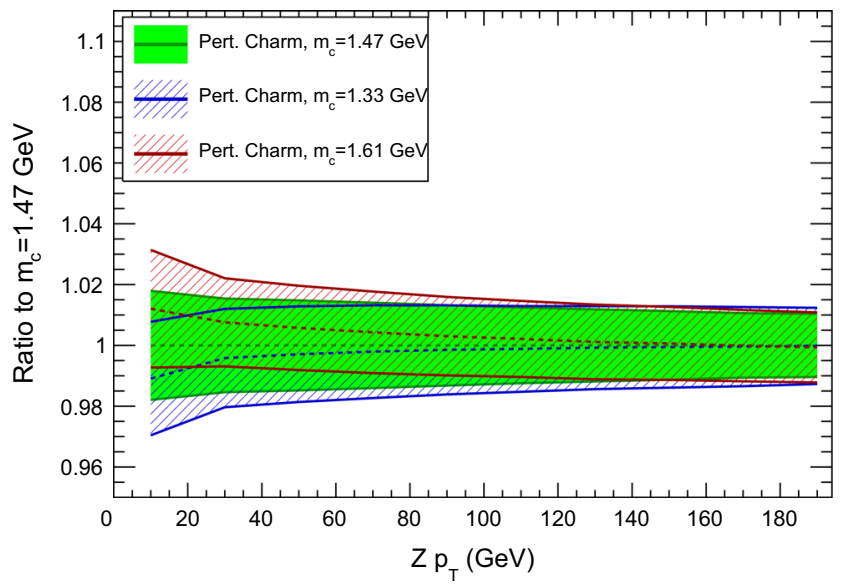

Inclusive W production, LHC $13 \mathrm{TeV}$

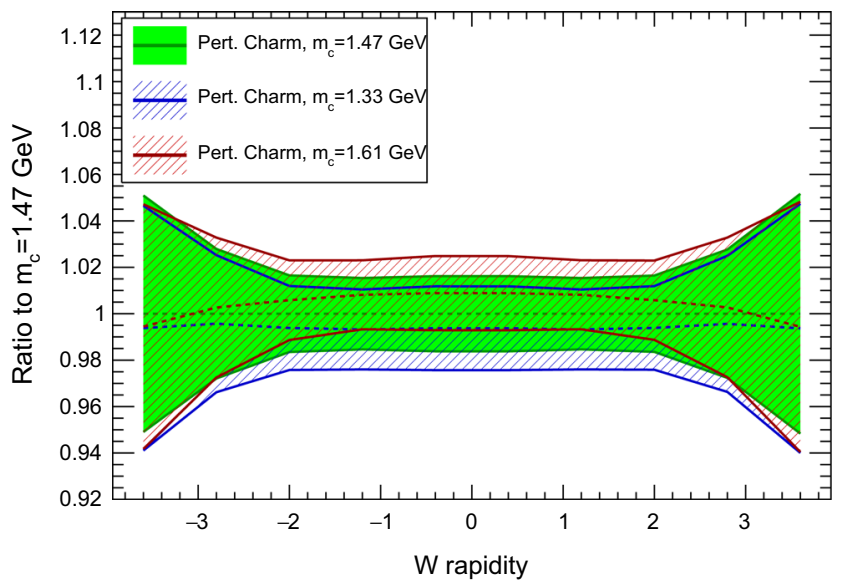

Fig. 28 Same as Fig. 27 for the $p_{T}$ of the $Z$ boson and the rapidity of the $W$ boson

Fig. 29 Representative leading-order Feynman diagrams for the production of a $Z$ boson in association with a charm quark at hadron colliders
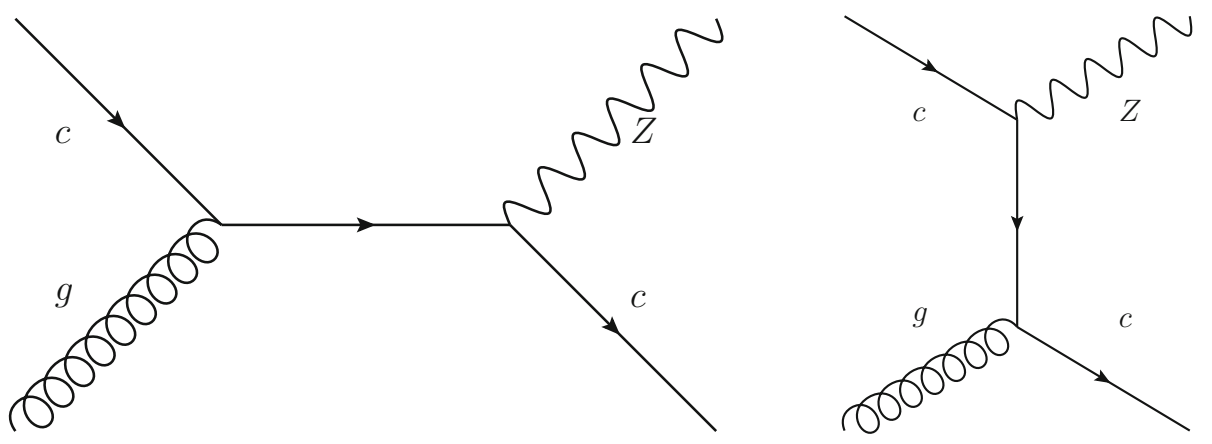

\subsubsection{Charm quark pair production}

At hadron colliders, heavy quark pair production is driven by the $g g$ and $q \bar{q}$ luminosities. The relative importance of the two channels depends on the kinematics. For instance, for the total inclusive cross section in top quark pair production [122], the $g g$ process is dominant at the LHC $13 \mathrm{TeV}$ (90\%), while it is only $14 \%$ at the Tevatron (where instead $86 \%$ of the cross section comes from quark-initiated contributions). In the case of charm quark pair production, at low transverse momentum $p_{T}^{c}$, the cross section is entirely dominated by gluon-initiated processes [91]. However, in the case of fitted charm the $c \bar{c}$ channel can eventually become dominant for high enough transverse momentum of the charm quark $p_{T}^{c}$, or for high enough rapidity $y_{c}$ : in these cases, large values of $x$ are probed, where the fall-off of the charm PDF is less steep than that of the gluon, especially if charm has an intrinsic component. Representative leading-order Feynman diagrams for the production of a charm-anticharm pair at hadron colliders are shown in Fig. 31. 


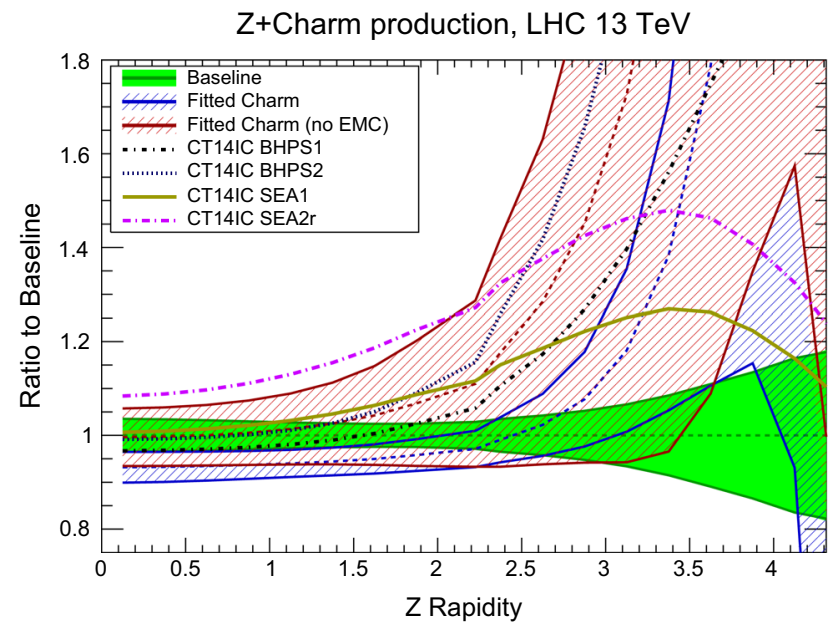

Fig. 30 The $Z$ boson rapidity (left) and transverse momentum (right) distributions for $Z$ production in association with charm at the LHC 13 $\mathrm{TeV}$, computed using the NNPDF sets with perturbative or fitted charm,
Fig. 31 Representative leading-order Feynman charm-anticharm pair at hadron colliders, initiated either by charm quarks (left) and by gluons (right) diagrams for the production of a

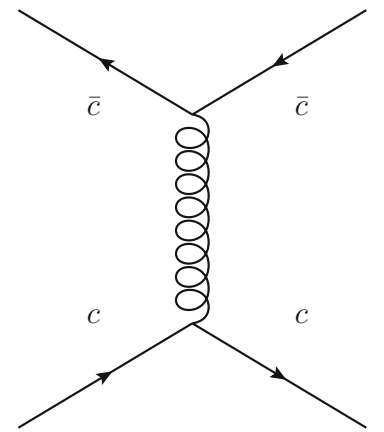

In the following, we use the FONLL code [18] for the calculation of the double-differential cross section $\mathrm{d}^{2} \sigma_{c \bar{c}} / \mathrm{d} p_{T} \mathrm{~d} y$ for the production of a charm-anticharm pair at hadron colliders. The FONLL calculation combines a fixed-order massive result, accurate at small $p_{T}$, with a resummed next-toleading log prediction in which the charm mass is neglected. As in the case of deep-inelastic scattering, the massive fixedorder calculation should be modified in the presence of a fitted charm component [22,23]. This modification is not included in the code of Ref. [18]; here, however, we will only consider the large $p_{T} \gg m_{c}$ region, where the FONLL computation coincides with the massless one and this extra contribution is negligible. Since our aim is only to illustrate how differences in the charm PDF affect the charm pair production cross section, we do not include final-state effects such as hadronization of charm quarks into $D$ mesons and their subsequent decay.

In Fig. 32 we show the double-differential cross section $d^{2} \sigma_{c \bar{c}} / d p_{T} d y$ for charm-anticharm pair production at the LHC $13 \mathrm{TeV}$, as a function of the charm quark transverse momentum for different values of its rapidity $y_{c}$. The impact of different charm PDFs becomes more important at large $p_{T}^{c}$ and for large $y_{c}$. For instance, for $y_{c}=3.5$, intrinsic charm

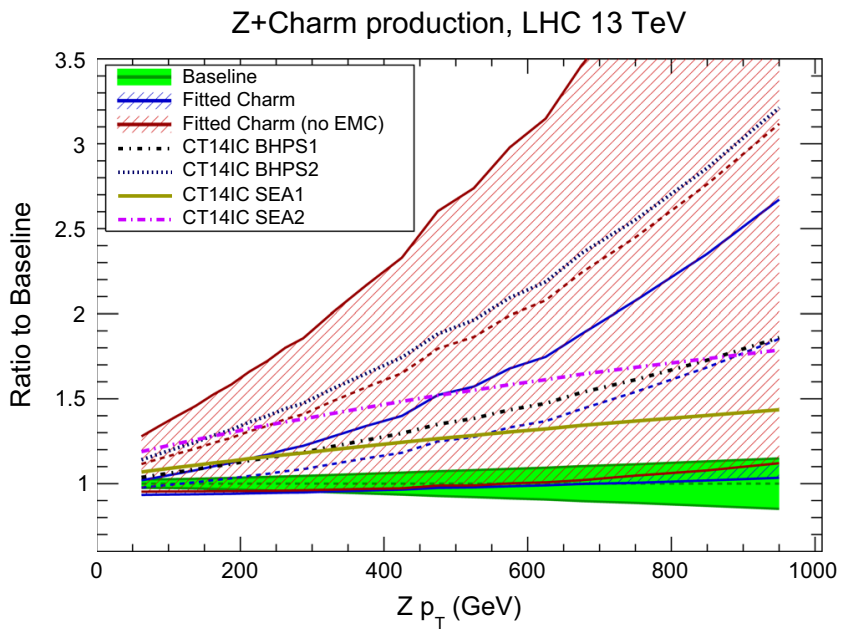

and the CT14 IC PDFs shown in Fig. 19. Results are shown as a ratio to the NNPDF perturbative charm set

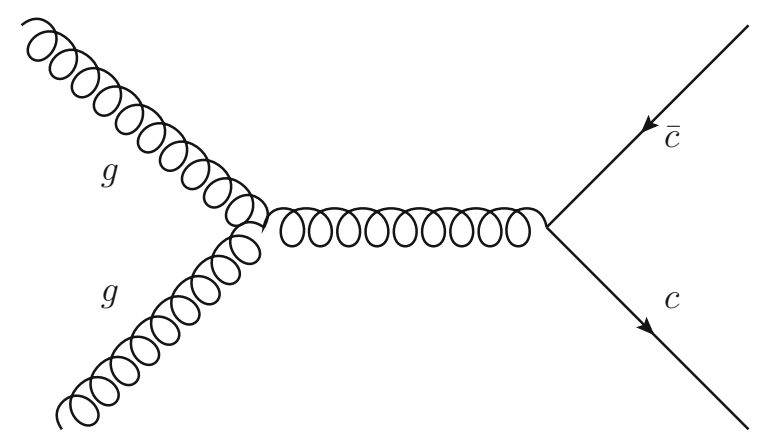

can enhance the cross section for charm production by up to one order of magnitude for $p_{T}^{c}=200 \mathrm{GeV}$.

While $D$ meson production in the forward region has been measured by LHCb, available data only cover the kinematic region up to $p_{T}^{D}=8 \mathrm{GeV}$ at $7 \mathrm{TeV}$ [92] and up $15 \mathrm{GeV}$ at $13 \mathrm{TeV}$ [123], where the differences between the fitted and perturbative charm predictions are small. Future $\mathrm{LHCb}$ $D$ meson production data with higher integrated luminosity and a higher reach in $p_{T}^{D}$ could be used to constrain the charm content of the proton. Similarly, $D$ meson production in the central region $\left|\eta_{D}\right| \lesssim 2$, but higher $p_{T}^{D}$ values than currently available could provide valuable constraints. Note that likewise current ATLAS $D$ meson measurements at 7 $\mathrm{TeV}$ [124] extend only up to $p_{T}^{D}=100 \mathrm{GeV}$, so data at 13 $\mathrm{TeV}$ with increased luminosity would also be required here.

\section{Delivery and outlook}

We have presented a first model-independent determination of the charm content of the proton in the NNPDF framework. Our results suggest that, if the EMC data are taken at face value, the charm PDF is compatible with perturba- 


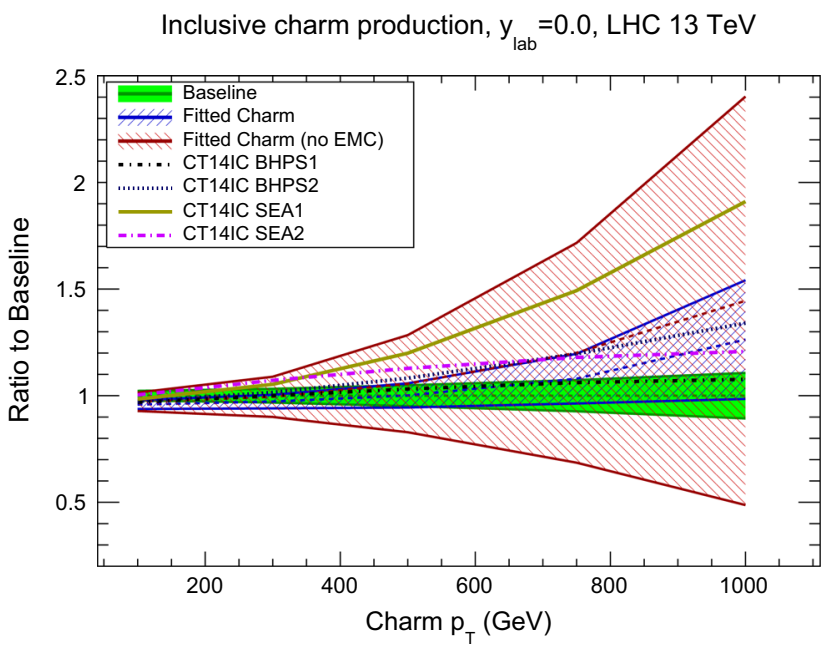

Inclusive charm production, $\mathrm{y}_{\mathrm{lab}}=2.0, \mathrm{LHC} 13 \mathrm{TeV}$

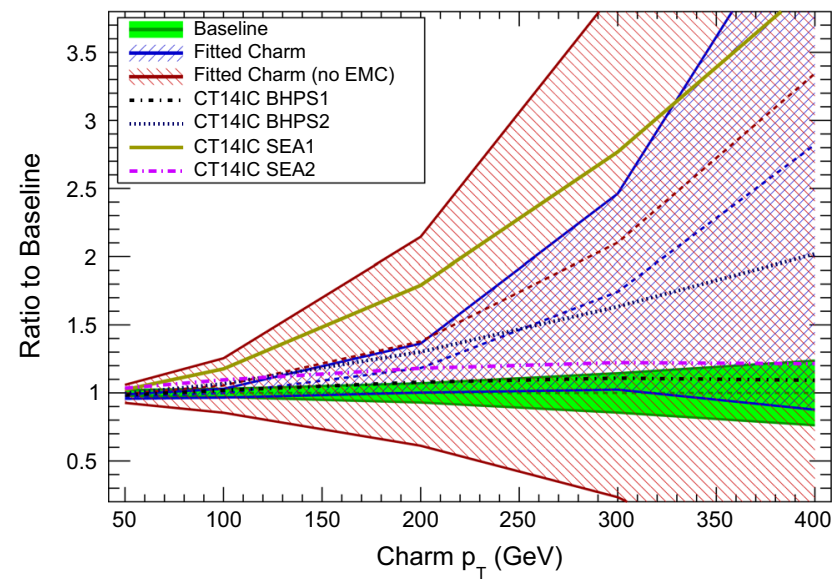

Fig. 32 The double-differential cross section $\mathrm{d}^{2} \sigma_{c \bar{c}} / \mathrm{d} p_{T} \mathrm{~d} y$ for charm-anticharm pair production at the LHC $13 \mathrm{TeV}$, as a function of the charm quark $p_{T}$ for different values of its rapidity $y$. From top to bottom and from left to right, we show the results for $y_{c}=0,1.0,2.0$

tive behaviour for $x \lesssim 0.1$, in that it vanishes for all $x$ in this region around $Q_{0} \approx 1.6 \mathrm{GeV}$, while it has an 'intrinsic' large $x$ component which peaks for $x \sim 0.5$, and carries $0.7 \pm 0.3 \%$ of the nucleon momentum at the $68 \% \mathrm{CL}$ at a low scale $Q=1.65 \mathrm{GeV}$. The perturbative component of our fitted charm is quite stable upon variation of the charm mass, and thus lies significantly below perturbatively generated charm if the central PDF value $m_{c}=1.47 \mathrm{GeV}$ is adopted. This could possibly be due to missing higher-order corrections, which are expected to be of comparable size. This suggests that PDF sets (including NNPDF3.0), in which charm is perturbatively generated but no theoretical uncertainties are provided, may be significantly underestimating the uncertainty on the charm PDF at small $x$, and missing its intrinsic component at large $x$. These results hold even if the uncertainty on the EMC charm data is considerably inflated,

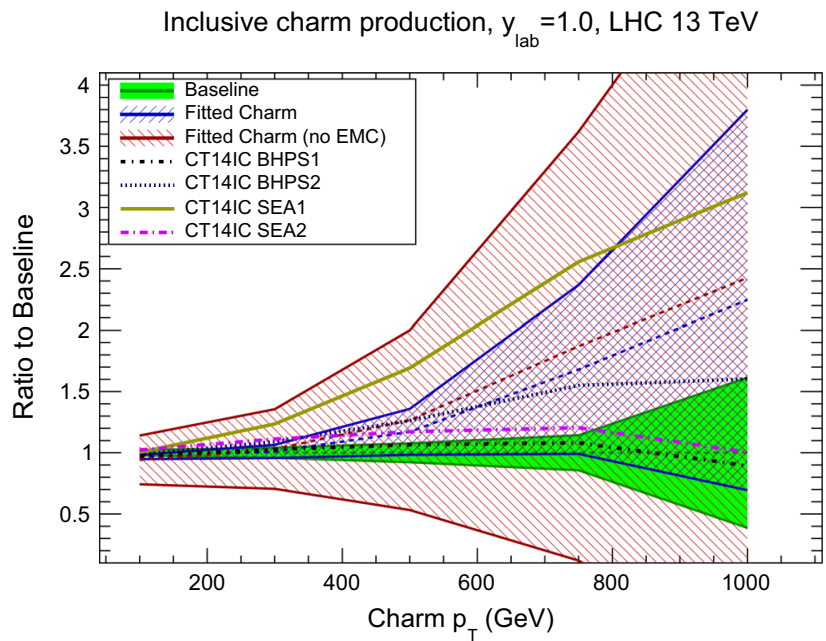

Inclusive charm production, $\mathrm{y}_{\mathrm{lab}}=3.5, \mathrm{LHC} 13 \mathrm{TeV}$

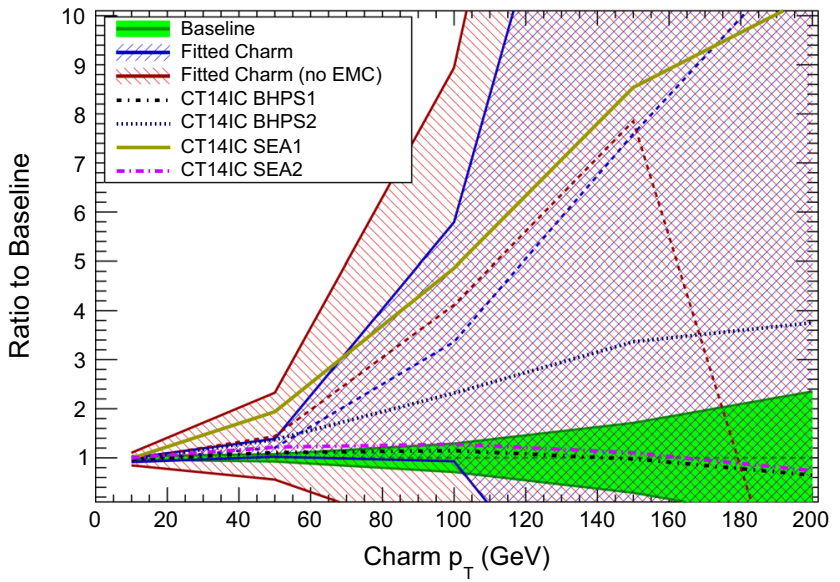

and 3.5. We compare results obtained using the NNPDF sets with perturbative or fitted charm, and the CT14 IC PDFs shown in Fig. 19. Results are shown as a ratio to the NNPDF perturbative charm set

and in fact at the level of central values they still hold even with the EMC data excluded altogether, though in that case they lose statistical significance.

Perhaps more interestingly, our results show that the widely held opinion (see e.g. Ref. [14] and Refs. therein) that the EMC data cannot be included in a global fit because they are in tension with other datasets, i.e. they cannot be adequately fit at leading-twist taking both data and theory at face value, is untenable. Indeed, we show that if we take the published EMC $F_{2}^{c}$ data and simply include them in an NLO fit based on the FONLL-B scheme with a fitted charm PDF we can fit them perfectly, with a $\chi^{2}$ per data point equal to $\chi^{2} / N_{\text {dat }}=1.09$. In other words, regardless of their reliability, the EMC data provide us with an interesting test-case scenario which demonstrates that the perturbative treatment of charm in current PDF fits may fail to satisfy the accu- 
racy standards that are required in order to match the high precision that current PDF uncertainties suggest.

When charm is fitted on the same footing as the other light PDFs, we find a small but non-negligible general improvement in global fit quality, and a very significant improvement in the description of large $x$ charm structure function data from EMC, which cannot be fitted otherwise. The dependence of the charm PDF on the value of the charm mass is significantly reduced, and there is also a more modest reduction in the charm mass dependence of light quark PDFs. We also find that, while with fitted charm overall uncertainties on gluon-induced LHC cross sections are a little more conservative, the charm mass dependence of quark-induced processes can be reduced at central rapidity and low $p_{T}$. This suggests that the fitted charm PDFs will lead to more reliable phenomenology at the LHC, eliminating a possible source of bias from assumptions as regards the origin of charm and the value of the charm mass.

An immediate consequence of our results is that existing determinations of the charm quark mass from deep-inelastic structure functions [24,73,104,125-127] might be affected by underestimated theory uncertainties due to the assumption that charm is generated perturbatively. With this motivation, we plan to perform in the near future a direct determination of the charm mass in the global NNPDF analysis both with fitted and with perturbative charm, using the same approach as for the determination of the strong coupling constant $[84,85]$.

Inclusion of a fitted charm PDF is planned for future general-purpose global PDF sets from the NNPDF Collaboration. Further measurements which might constrain the fitted charm, in particular $Z+c$ and $c \bar{c}$ production at high $p_{T}$ and high rapidity, are expected at LHC Run 2 . We expect the accuracy of the charm determination to improve substantially in the near future, and the issue of the reliability of the EMC data to be finally settled by these measurements.

The NLO PDFs presented here are available in the LHAPDF 6 format [128] from the NNPDF HepForge webpage:

https://nnpdf.hepforge.org/html/nnpdf3ic/nnpdf3ic.html In particular, we make available the following PDF sets:

- PDF sets with fitted charm, for three different values of the pole charm mass:

NNPDF3_IC_nlo_as_0118_mcpole_1330

NNPDF3_IC_nlo_as_0118_mcpole_1470

NNPDF3_IC_nlo_as_0118_mcpole_1610

- PDF sets with identical theory settings as those above, with the only differences being that the charm PDF is perturbatively generated and that the EMC data are excluded, for the same three values of the charm mass:

NNPDF3_nIC_nlo_as_0118_mcpole_1330

NNPDF3_nIC_nlo_as_0118_mcpole_1470

NNPDF3_nIC_nlo_as_0118_mcpole_1610
- A PDF set with fitted charm and the central value of the charm quark pole mass $m_{c}^{\text {pole }}=1.47 \mathrm{GeV}$ without the EMC charm data included:

NNPDF3_IC_nlo_as_0118_mcpole_1470_noE

$\mathrm{MC}$

- PDF sets with fitted charm, for three different values of the running $\overline{\mathrm{MS}}$ charm mass:

$$
\begin{aligned}
& \text { NNPDF3_IC_nlo_as_0118_mc_1150 } \\
& \text { NNPDF3_IC_n1o_as_0118_mC_1275 } \\
& \text { NNPDF3_IC_nlo_as_0118_mC_1400 }
\end{aligned}
$$

- PDF sets with identical theory settings as those above, with the only differences being that the charm PDF is perturbatively generated and that the EMC data are excluded, for the same three values of the charm mass:

$$
\begin{aligned}
& \text { NNPDF3_nIC_n1o_as_0118_mc_1150 } \\
& \text { NNPDF3_nIC_n1o_as_0118_mc_1275 } \\
& \text { NNPDF3_nIC_nlo_as_0118_mc_1400 }
\end{aligned}
$$

- A PDF set with fitted charm and central theory settings but without the EMC charm data included:

NNPDF3_IC_n1o_as_0118_mc_1275_noEMC

These PDF sets are not meant to be used for generalpurpose applications, for which the NNPDF3.0 PDF sets are still recommended, but rather for studies related to the charm content of the proton. Specifically, fitted charm PDFs should always be compared with the corresponding baseline fits presented in this publication, in order to have a consistent comparison of two PDF sets with identical theory and methodological settings and only differing in the treatment of charm and the inclusion or not of the EMC charm data.

Acknowledgements We thank J. Feltesse and A. Cooper-Sarkar for comments on the EMC data, P. Nadolsky and R. Thorne for several stimulating comments on many aspects of our results, and especially M. Arneodo for detailed information on the EMC charm structure function data. RDB is funded by an STFC Consolidated Grant ST/J000329/1; SC is supported by the HICCUP ERC Consolidator Grant (614577); SF is supported by the Executive Research Agency (REA) of the European Commission under the Grant Agreement PITNGA-2012-316704 (HiggsTools); JR is supported by an STFC Rutherford Fellowship and Grant ST/K005227/1 and ST/M003787/1; VB, $\mathrm{MB}, \mathrm{NH}$, JR and LR are supported by an European Research Council Starting Grant "PDF4BSM".

Open Access This article is distributed under the terms of the Creative Commons Attribution 4.0 International License (http://creativecomm ons.org/licenses/by/4.0/), which permits unrestricted use, distribution, and reproduction in any medium, provided you give appropriate credit to the original author(s) and the source, provide a link to the Creative Commons license, and indicate if changes were made. Funded by SCOAP . $^{3}$

\section{References}

1. NNPDF Collaboration, R.D. Ball et al., Parton distributions for the LHC Run II. JHEP 04, 040 (2015). arXiv:1410.8849 
2. S. Dulat, T.-J. Hou, J. Gao, M. Guzzi, J. Huston, P. Nadolsky, J. Pumplin, C. Schmidt, D. Stump, C.P. Yuan, New parton distribution functions from a global analysis of quantum chromodynamics. Phys. Rev. D 93(3), 033006 (2016). arXiv:1506.07443

3. L.A. Harland-Lang, A.D. Martin, P. Motylinski, R.S. Thorne, Parton distributions in the LHC era: MMHT 2014 PDFs. Eur. Phys. J. C 75, 204 (2015). arXiv: 1412.3989

4. S. Alekhin, J. Blümlein, S. Moch, The ABM parton distributions tuned to LHC data. Phys. Rev. D 89, 054028 (2014). arXiv: 1310.3059

5. ZEUS, $\mathrm{H} 1$ Collaboration, $\mathrm{H}$. Abramowicz et al., Combination of measurements of inclusive deep inelastic $e^{ \pm} p$ scattering cross sections and QCD analysis of HERA data. Eur. Phys. J. C 75(12), 580 (2015). arXiv: 1506.06042

6. A. Accardi, L.T. Brady, W. Melnitchouk, J.F. Owens, N. Sato, Constraints on large- $x$ parton distributions from new weak boson production and deep-inelastic scattering data. arXiv:1602.03154

7. P. Jimenez-Delgado, E. Reya, Delineating parton distributions and the strong coupling. Phys. Rev. D 89(7), 074049 (2014). arXiv: 1403.1852

8. S.J. Brodsky, A. Kusina, F. Lyonnet, I. Schienbein, H. Spiesberger, R. Vogt, A review of the intrinsic heavy quark content of the nucleon. Adv. High Energy Phys. 2015, 231547 (2015). arXiv: 1504.06287

9. J.C. Collins, Hard scattering factorization with heavy quarks: a General treatment. Phys. Rev. D 58, 094002 (1998). arXiv:hep-ph/9806259

10. B.W. Harris, J. Smith, R. Vogt, Reanalysis of the EMC charm production data with extrinsic and intrinsic charm at NLO. Nucl. Phys. B 461, 181-196 (1996). arXiv:hep-ph/9508403

11. J. Pumplin, H.L. Lai, W.K. Tung, The charm parton content of the nucleon. Phys. Rev. D 75, 054029 (2007). arXiv:hep-ph/0701220

12. A.D. Martin, W.J. Stirling, R.S. Thorne, G. Watt, Parton distributions for the LHC. Eur. Phys. J. C 63, 189 (2009). arXiv:0901.0002

13. S. Dulat et al., Intrinsic charm parton distribution functions from CTEQ-TEA global analysis. Phys. Rev. D 89(7), 073004 (2014). arXiv: 1309.0025

14. P. Jimenez-Delgado, T. Hobbs, J. Londergan, W. Melnitchouk, New limits on intrinsic charm in the nucleon from global analysis of parton distributions. Phys. Rev. Lett. 114(8), 082002 (2015). arXiv: 1408.1708

15. T.-J. Hou, S. Dulat, J. Gao, M. Guzzi, J.W. Huston, P. Nadolsky, J. Pumplin, C.R. Schmidt, D. Stump, C.P. Yuan, Heavy flavors on CT14. PoS DIS2015,166 (2015)

16. E. Hoffmann, R. Moore, Subleading contributions to the intrinsic charm of the nucleon. Z. Phys. C 20, 71 (1983)

17. S. Kretzer, I. Schienbein, Heavy quark initiated contributions to deep inelastic structure functions. Phys. Rev. D 58, 094035 (1998). arXiv:hep-ph/9805233

18. M. Cacciari, M. Greco, P. Nason, The $p(T)$ spectrum in heavy-flavour hadroproduction. JHEP 05, 007 (1998). arXiv:hep-ph/9803400

19. S. Forte, E. Laenen, P. Nason, J. Rojo, Heavy quarks in deep-inelastic scattering. Nucl. Phys. B 834, 116-162 (2010). arXiv: 1001.2312

20. M. Kramer, F.I. Olness, D.E. Soper, Treatment of heavy quarks in deeply inelastic scattering. Phys. Rev. D 62, 096007 (2000). arXiv:hep-ph/0003035

21. R. Vogt, S.J. Brodsky, Intrinsic charm contribution to double quarkonium hadroproduction. Phys. Lett. B 349, 569-575 (1995). arXiv:hep-ph/9503206

22. R.D. Ball, V. Bertone, M. Bonvini, S. Forte, P. Groth Merrild, J. Rojo, L. Rottoli, Intrinsic charm in a matched general-mass scheme. Phys. Lett. B 754, 49-58 (2016). arXiv:1510.00009

23. R.D. Ball, M. Bonvini, L. Rottoli, Charm in deep-inelastic scattering. JHEP 11, 122 (2015). arXiv:1510.02491
24. H1, ZEUS Collaboration, H. Abramowicz et al., Combination and QCD analysis of charm production cross section measurements in deep-inelastic ep scattering at HERA. Eur. Phys. J. C 73, 2311 (2013). arXiv:1211.1182

25. European Muon Collaboration, J.J. Aubert et al., Production of charmed particles in $250-\mathrm{GeV} \mu^{+}$-iron interactions. Nucl. Phys. B 213, 31 (1983)

26. H1 and ZEUS Collaboration, F. Aaron et al., Combined measurement and QCD analysis of the inclusive $e^{ \pm} p$ scattering cross sections at HERA. JHEP 1001, 109 (2010). arXiv:0911.0884

27. H1 Collaboration, F. Aaron et al., Inclusive deep inelastic scattering at high $Q^{2}$ with longitudinally polarised lepton beams at HERA. JHEP 1209, 061 (2012). arXiv:1206.7007

28. H1 Collaboration, F. Aaron et al., Measurement of the inclusive $e^{ \pm} p$ scattering cross section at high inelasticity $y$ and of the structure function $F_{L}$. Eur. Phys. J. C 71, 1579 (2011). arXiv: 1012.4355

29. ZEUS Collaboration, H. Abramowicz, Measurement of high$Q^{2}$ neutral current deep inelastic $e^{+} p$ scattering cross sections with a longitudinally polarised positron beam at HERA. arXiv: 1208.6138

30. Z.E.U.S. Collaboration, H. Abramowicz et al., Measurement of high- $Q^{2}$ charged current deep inelastic scattering cross sections with a longitudinally polarised positron beam at HERA. Eur. Phys. J. C 70, 945-963 (2010). arXiv: 1008.3493

31. J. Rojo, Progress in the NNPDF global analysis and the impact of the legacy HERA combination. PoS EPS-HEP2015, 506 (2015). arXiv: 1508.07731

32. L.A. Harland-Lang, A.D. Martin, P. Motylinski, R.S. Thorne, The impact of the final HERA combined data on PDFs obtained from a global fit. Eur. Phys. J. C 76(4), 186 (2016). arXiv: 1601.03413

33. S.J. Brodsky, P. Hoyer, A.H. Mueller, W.-K. Tang, New QCD production mechanisms for hard processes at large $x$. Nucl. Phys. B 369, 519-542 (1992)

34. New Muon Collaboration, M. Arneodo et al., Accurate measurement of $F_{2}^{d} / F_{2}^{p}$ and $R_{d}-R_{p}$. Nucl. Phys. B 487, 3-26 (1997). arXiv:hep-ex/9611022

35. New Muon Collaboration, M. Arneodo et al., Measurement of the proton and deuteron structure functions, $F_{2}^{p}$ and $F_{2}^{d}$, and of the ratio $\sigma_{L} / \sigma_{T}$. Nucl. Phys. B 483, 3-43 (1997). arXiv:hep-ph/9610231

36. BCDMS Collaboration, A.C. Benvenuti et al., A high statistics measurement of the proton structure functions $f_{2}\left(x, q^{2}\right)$ and $r$ from deep inelastic muon scattering at high $q^{2}$. Phys. Lett. B 223, 485 (1989)

37. BCDMS Collaboration, A.C. Benvenuti et al., A high statistics measurement of the deuteron structure functions $f_{2}\left(x, q^{2}\right)$ and $r$ from deep inelastic muon scattering at high $q^{2}$. Phys. Lett. B 237, 592 (1990)

38. L.W. Whitlow, E.M. Riordan, S. Dasu, S. Rock, A. Bodek, Precise measurements of the proton and deuteron structure functions from a global analysis of the SLAC deep inelastic electron scattering cross-sections. Phys. Lett. B 282, 475-482 (1992)

39. CHORUS Collaboration, G. Onengut et al., Measurement of nucleon structure functions in neutrino scattering. Phys. Lett. B 632, 65-75 (2006)

40. NuTeV Collaboration, M. Goncharov et al., Precise measurement of dimuon production cross-sections in $v_{\mu} \mathrm{Fe}$ and $\bar{v}_{\mu} \mathrm{Fe}$ deep inelastic scattering at the Tevatron. Phys. Rev. D 64, 112006 (2001). arXiv:hep-ex/0102049

41. D.A. Mason, Measurement of the strange-antistrange asymmetry at NLO in QCD from NuTeV dimuon data. FERMILAB-THESIS2006-01

42. G. Moreno et al., Dimuon production in proton-copper collisions at $\sqrt{s}=38.8-G e V$. Phys. Rev. D 43, 2815-2836 (1991) 
43. NuSea Collaboration, J.C. Webb et al., Absolute Drell-Yan dimuon cross sections in $800-\mathrm{GeV} / \mathrm{c}$ pp and pd collisions. arXiv:hep-ex/0302019

44. J.C. Webb, Measurement of continuum dimuon production in 800$\mathrm{GeV} / \mathrm{c}$ proton nucleon collisions. arXiv:hep-ex/0301031

45. FNAL E866/NuSea Collaboration, R.S. Towell et al., Improved measurement of the anti-d/anti-u asymmetry in the nucleon sea. Phys. Rev. D 64, 052002 (2001). arXiv:hep-ex/0103030

46. C.D.F. Collaboration, T.A. Aaltonen et al., Measurement of $d \sigma / d y$ of Drell-Yan $e^{+} e^{-}$pairs in the $Z$ mass region from $p \bar{p}$ collisions at $\sqrt{s}=1.96$ TeV. Phys. Lett. B 692, 232-239 (2010). arXiv:0908.3914

47. D0 Collaboration, V.M. Abazov et al., Measurement of the shape of the boson rapidity distribution for $p \bar{p} \rightarrow Z / \gamma^{*} \rightarrow e^{+} e^{-}+$ $X$ events produced at $\sqrt{s}=1.96-\mathrm{TeV}$. Phys. Rev. D 76, 012003 (2007). arXiv:hep-ex/0702025

48. C.D.F. Collaboration, T. Aaltonen et al., Measurement of the Inclusive Jet Cross Section at the Fermilab Tevatron p-pbar Collider Using a Cone-Based Jet Algorithm. Phys. Rev. D 78, 052006 (2008). arXiv:0807.2204

49. ATLAS Collaboration, G. Aad et al., Measurement of the inclusive $W^{ \pm}$and $Z / \gamma^{*}$ cross sections in the electron and muon decay channels in pp collisions at $\sqrt{s}=7 \mathrm{TeV}$ with the ATLAS detector. Phys.Rev. D 85 (2012) 072004, arXiv:1109.5141

50. ATLAS Collaboration, G. Aad et al., Measurement of the highmass Drell-Yan differential cross-section in pp collisions at $\sqrt{s}$ $=7 \mathrm{TeV}$ with the ATLAS detector. Phys. Lett. B 725, 223 (2013). arXiv: 1305.4192

51. ATLAS Collaboration, G. Aad et al., Measurement of the transverse momentum distribution of momentum distribution of $W$ bosons in $p p$ collisions at $\sqrt{s}=7 \mathrm{TeV}$ with the ATLAS detector. Phys. Rev. D 85, 012005 (2012). arXiv: 1108.6308

52. C.M.S. Collaboration, S. Chatrchyan et al., Measurement of the electron charge asymmetry in inclusive $\mathrm{W}$ production in pp collisions at $\sqrt{s}=7$ TeV. Phys. Rev. Lett. 109, 111806 (2012). arXiv: 1206.2598

53. C.M.S. Collaboration, S. Chatrchyan et al., Measurement of the muon charge asymmetry in inclusive pp to WX production at $\sqrt{s}=$ $7 \mathrm{TeV}$ and an improved determination of light parton distribution functions. Phys. Rev. D 90, 032004 (2014). arXiv:1312.6283

54. C.M.S. Collaboration, S. Chatrchyan et al., Measurement of associated $\mathrm{W}+$ charm production in pp collisions at $\sqrt{s}=7 \mathrm{TeV}$. JHEP 02, 013 (2014). arXiv: 1310.1138

55. C.M.S. Collaboration, S. Chatrchyan et al., Measurement of the differential and double-differential Drell-Yan cross sections in proton-proton collisions at $\sqrt{s}=7 \mathrm{TeV}$. JHEP 1312, 030 (2013). arXiv: 1310.7291

56. LHCb Collaboration, R. Aaij et al., Inclusive $W$ and $Z$ production in the forward region at $\sqrt{s}=7 \mathrm{TeV}$. JHEP 1206, 058 (2012). arXiv: 1204.1620

57. LHCb Collaboration, R. Aaij et al., Measurement of the crosssection for $Z \rightarrow e^{+} e^{-}$production in $p p$ collisions at $\sqrt{s}=7$ TeV. JHEP 1302, 106 (2013). arXiv: 1212.4620

58. ATLAS Collaboration, G. Aad et al., Measurement of inclusive jet and dijet production in pp collisions at $\sqrt{s}=7 \mathrm{TeV}$ using the ATLAS detector. Phys. Rev. D 86, 014022 (2012). arXiv: 1112.6297

59. ATLAS Collaboration, G. Aad et al., Measurement of the inclusive jet cross section in pp collisions at $\sqrt{s}=2.76 \mathrm{TeV}$ and comparison to the inclusive jet cross section at $\sqrt{s}=7 \mathrm{TeV}$ using the ATLAS detector. Eur. Phys. J. C 73, 2509 (2013). arXiv:1304.4739

60. C.M.S. Collaboration, S. Chatrchyan et al., Measurements of differential jet cross sections in proton-proton collisions at $\sqrt{s}=7$ $\mathrm{TeV}$ with the CMS detector. Phys. Rev. D 87, 112002 (2013). arXiv: 1212.6660
61. ATLAS Collaboration, G. Aad et al., Measurement of the cross section for top-quark pair production in $p p$ collisions at $\sqrt{s}=7$ $\mathrm{TeV}$ with the ATLAS detector using final states with two high-pt leptons. JHEP 1205, 059 (2012). arXiv: 1202.4892

62. ATLAS Collaboration, G. Aad et al., Measurement of the $t \bar{t}$ production cross-section in pp collisions at sqrts $=7 \mathrm{TeV}$ using kinematic information of lepton+jets events. ATLAS-CONF-2011121, ATLAS-COM-CONF-2011-132

63. ATLAS Collaboration, G. Aad et al., Measurement of the $t \bar{t}$ production cross-section in $p p$ collisions at $\sqrt{s}=8 \mathrm{TeV}$ using $e \mu$ events with $b$-tagged jets. ATLAS-CONF-2013-097, ATLASCOM-CONF-2013-112

64. C.M.S. Collaboration, S. Chatrchyan et al., Measurement of the $t \bar{t}$ production cross section in the dilepton channel in pp collisions at $\sqrt{s}=8$ TeV. JHEP 1402, 024 (2014). arXiv: 1312.7582

65. C.M.S. Collaboration, S. Chatrchyan et al., Measurement of the $t \bar{t}$ production cross section in the dilepton channel in $p p$ collisions at $\sqrt{s}=7$ TeV. JHEP 1211, 067 (2012). arXiv:1208.2671

66. C.M.S. Collaboration, S. Chatrchyan et al., Measurement of the $t \bar{t}$ production cross section in $p p$ collisions at $\sqrt{s}=7 \mathrm{TeV}$ with lepton + jets final states. Phys. Lett. B 720, 83-104 (2013). arXiv: 1212.6682

67. M. Bonvini, S. Marzani, J. Rojo, L. Rottoli, M. Ubiali, R.D. Ball, V. Bertone, S. Carrazza, N.P. Hartland, Parton distributions with threshold resummation. JHEP 09, 191 (2015). arXiv:1507.01006

68. The NNPDF Collaboration, R.D. Ball et al., A determination of parton distributions with faithful uncertainty estimation. Nucl. Phys. B 809, 1-63 (2009). arXiv:0808.1231

69. The NNPDF Collaboration, R.D. Ball et al., A first unbiased global NLO determination of parton distributions and their uncertainties. Nucl. Phys. B 838, 136 (2010). arXiv: 1002.4407

70. V. Bertone, S. Carrazza, J. Rojo, APFEL: A PDF evolution library with QED corrections. Comput. Phys. Commun. 185, 1647 (2014). arXiv:1310.1394

71. T. Carli et al., A posteriori inclusion of parton density functions in NLO QCD final-state calculations at hadron colliders: The APPLGRID Project. Eur. Phys. J. C 66, 503 (2010). arXiv:0911.2985

72. V. Bertone, S. Carrazza, N.P. Hartland, APFELgrid: a high performance tool for parton density determinations. arXiv: 1605.02070

73. xFitter Developers' Team Collaboration, V. Bertone et al., A determination of $\mathrm{mc}(\mathrm{mc})$ from HERA data using a matched heavyflavor scheme. arXiv: 1605.01946

74. S. Alekhin, S. Moch, Heavy-quark deep-inelastic scattering with a running mass. Phys. Lett. B 699, 345-353 (2011). arXiv: 1011.5790

75. G.P. Salam, J. Rojo, A higher order perturbative parton evolution toolkit (HOPPET). Comput. Phys. Commun. 180, 120-156 (2009). arXiv:0804.3755

76. http://www.ge.infn.it/ bonvini/massivedis/

77. J. Particle, Data Group, Beringer et al., Review of particle physics (RPP). Phys. Rev. D 86, 010001 (2012)

78. E.R. Nocera, Small- and large- $x$ nucleon spin structure from a global QCD analysis of polarized parton distribution functions. Phys. Lett. B 742, 117-125 (2015). arXiv:1410.7290

79. R.D. Ball, E.R. Nocera, J. Rojo, The asymptotic behaviour of parton distributions at small and large $x$. arXiv:1604.00024

80. R.D. Ball, V. Bertone, F. Cerutti, L. Del Debbio, S. Forte, A. Guffanti, J.I. Latorre, J. Rojo, M. Ubiali, Impact of heavy quark masses on parton distributions and LHC phenomenology. Nucl. Phys. B 849, 296-363 (2011). arXiv: 1101.1300

81. NNPDF Collaboration, R.D. Ball et al., Parton distributions: determining probabilities in a space of functions. arXiv: 1110.1863

82. The NNPDF Collaboration, R.D. Ball et al., Fitting parton distribution data with multiplicative normalization uncertainties. JHEP 05, 075 (2010). arXiv:0912.2276 
83. R.D. Ball, S. Carrazza, L. Del Debbio, S. Forte, J. Gao et al., Parton distribution benchmarking with LHC data. JHEP 1304, 125 (2013). arXiv: 1211.5142

84. S. Lionetti et al., Precision determination of $\alpha_{s}$ using an unbiased global NLO parton set. Phys. Lett. B 701, 346-352 (2011). arXiv: 1103.2369

85. R.D. Ball, V. Bertone, L. Del Debbio, S. Forte, A. Guffanti et al., Precision NNLO determination of $\alpha_{s}\left(M_{Z}\right)$ using an unbiased global parton set. Phys. Lett. B 707, 66-71 (2012). arXiv: 1110.2483

86. J. Blümlein, A kinematic condition on intrinsic charm. Phys. Lett. B 753, 619-621 (2016). arXiv:1511.00229

87. J. Feltesse, Introduction to deep inelastic scattering: past and present. In: Proceedings, 20th International Workshop on DeepInelastic Scattering and Related Subjects (DIS 2012), pp. 3-12 (2012)

88. M. Arneodo, Private Communication

89. L. Rottoli, Fitting EMC structure functions with intrinsic charm. In: Proceedings, 24th International Workshop on Deep-Inelastic Scattering and Related Subjects (DIS 2016): Hamburg, Germany, April 11-25, 2016. arXiv:1606.09289

90. Particle Data Group Collaboration, K. Olive et al., Review of particle physics. Chin. Phys. C 38, 090001 (2014)

91. R. Gauld, J. Rojo, L. Rottoli, J. Talbert, Charm production in the forward region: constraints on the small- $x$ gluon and backgrounds for neutrino astronomy. JHEP 11, 009 (2015). arXiv:1506.08025

92. LHCb Collaboration, R. Aaij et al., Prompt charm production in pp collisions at sqrt(s)=7 TeV. Nucl. Phys. B 871, 1-20 (2013). arXiv: 1302.2864

93. K. Rith, Present status of the EMC effect. Subnucl. Ser. 51, 431449 (2015). arXiv: 1402.5000

94. BCDMS Collaboration, A.C. Benvenuti et al., A high statistics measurement of the proton structure functions $F_{2}\left(x, Q^{2}\right)$ and $R$ from deep inelastic muon scattering at high $Q^{2}$. Phys. Lett. B 223, 485 (1989)

95. BCDMS Collaboration, A.C. Benvenuti et al., A high statistics measurement of the deuteron structure functions $F_{2}\left(x, Q^{2}\right)$ and $R$ from deep inelastic muon scattering at high $Q^{2}$. Phys. Lett. B 237, 592 (1990)

96. M. Buza, Y. Matiounine, J. Smith, R. Migneron, W.L. van Neerven, Heavy quark coefficient functions at asymptotic values $Q 2 \gg m 2$. Nucl. Phys. B 472, 611-658 (1996). arXiv:hep-ph/9601302

97. M. Buza, Y. Matiounine, J. Smith, W.L. van Neerven, Charm electroproduction viewed in the variable-flavour number scheme versus fixed-order perturbation theory. Eur. Phys. J. C 1, 301-320 (1998). arXiv:hep-ph/9612398

98. S.J. Brodsky, P. Hoyer, C. Peterson, N. Sakai, The intrinsic charm of the proton. Phys. Lett. B 93, 451-455 (1980)

99. J.M. Campbell, J.W. Huston, W.J. Stirling, Hard interactions of quarks and gluons: A primer for LHC physics. Rept. Prog. Phys. 70, 89 (2007). arXiv:hep-ph/0611148

100. http://www.ge.infn.it/ bonvini/higgs/

101. M. Czakon, A. Mitov, Top++: A Program for the Calculation of the Top-Pair Cross-Section at Hadron Colliders. Comput. Phys. Commun. 185, 2930 (2014). [arXiv:1112.5675]

102. J. Campbell, R.K. Ellis, F. Tramontano, Single top production and decay at next-to-leading order. Phys. Rev. D 70, 094012 (2004). arXiv:hep-ph/0408158

103. A.D. Martin, W.J. Stirling, R.S. Thorne, G. Watt, Heavyquark mass dependence in global PDF analyses and 3- and 4flavour parton distributions. Eur. Phys. J. C 70, 51-72 (2010). arXiv: 1007.2624

104. J. Gao, M. Guzzi, P.M. Nadolsky, Charm quark mass dependence in a global QCD analysis. Eur. Phys. J. C 73, 2541 (2013). arXiv:1304.3494
105. L.A. Harland-Lang, A.D. Martin, P. Motylinski, R.S. Thorne, Charm and beauty quark masses in the MMHT2014 global PDF analysis. Eur. Phys. J. C 76(1), 10 (2016). arXiv:1510.02332

106. J. Alwall, R. Frederix, S. Frixione, V. Hirschi, F. Maltoni et al., The automated computation of tree-level and next-to-leading order differential cross sections, and their matching to parton shower simulations. JHEP 1407, 079 (2014). arXiv:1405.0301

107. V. Bertone, R. Frederix, S. Frixione, J. Rojo, M. Sutton, aMCfast: automation of fast NLO computations for PDF fits. JHEP 1408, 166 (2014). arXiv: 1406.7693

108. S. Carrazza, S. Forte, Z. Kassabov, J. Rojo, Specialized minimal PDFs for optimized LHC calculations. arXiv:1602.00005

109. T.P. Stavreva, J.F. Owens, Direct photon production in association with a heavy quark at hadron colliders. Phys. Rev. D 79, 054017 (2009). arXiv:0901.3791

110. S. Rostami, A. Khorramian, A. Aleedaneshvar, M. Goharipour, The impact of the intrinsic charm quark content of the proton on differential $\gamma+c$ cross section. arXiv: 1510.08421

111. V.A. Bednyakov, M.A. Demichev, G.I. Lykasov, T. Stavreva, M. Stockton, Searching for intrinsic charm in the proton at the LHC. Phys. Lett. B 728, 602-606 (2014). arXiv: 1305.3548

112. T. Boettcher, P. Ilten, M. Williams, A direct probe of the intrinsic charm content of the proton. Phys. Rev. D 93, 074008 (2016). arXiv: 1512.06666

113. G. Bailas, V.P. Goncalves, Phenomenological implications of the intrinsic charm in the $Z$ boson production at the LHC. Eur. Phys. J. C 76(3), 105 (2016). arXiv:1512.06007

114. P.-H. Beauchemin, V.A. Bednyakov, G.I. Lykasov, Y.Y. Stepanenko, Search for intrinsic charm in vector boson production accompanied by heavy flavor jets. Phys. Rev. D 92(3), 034014 (2015). arXiv:1410.2616

115. B.A. Kniehl, G. Kramer, I. Schienbein, H. Spiesberger, Inclusive $\mathrm{D}^{*}+-$ production in $\mathrm{p}$ anti-p collisions with massive charm quarks. Phys. Rev. D 71, 014018 (2005). arXiv:hep-ph/0410289

116. E.V. Bugaev, P.A. Klimai, About possible contribution of intrinsic charm component to inclusive spectra of charmed mesons. J. Phys. G 37, 055004 (2010). arXiv:0905.2309

117. R. Vogt, S.J. Brodsky, QCD and intrinsic heavy quark predictions for leading charm and beauty hadroproduction. Nucl. Phys. B 438, 261-277 (1995). arXiv:hep-ph/9405236

118. B.A. Kniehl, G. Kramer, I. Schienbein, H. Spiesberger, Open charm hadroproduction and the charm content of the proton. Phys. Rev. D 79, 094009 (2009). arXiv:0901.4130

119. B.A. Kniehl, G. Kramer, I. Schienbein, H. Spiesberger, Inclusive charmed-meson production at the CERN LHC. Eur. Phys. J. C 72, 2082 (2012). arXiv: 1202.0439

120. S.J. Brodsky, B. Kopeliovich, I. Schmidt, J. Soffer, Diffractive Higgs production from intrinsic heavy flavors in the proton. Phys. Rev. D 73, 113005 (2006). arXiv:hep-ph/0603238

121. S.J. Brodsky, A.S. Goldhaber, B.Z. Kopeliovich, I. Schmidt, Higgs hadroproduction at large Feynman $x$. Nucl. Phys. B 807, 334-347 (2009). arXiv:0707.4658

122. M. Czakon, M.L. Mangano, A. Mitov, J. Rojo, Constraints on the gluon PDF from top quark pair production at hadron colliders. JHEP 1307, 167 (2013). arXiv: 1303.7215

123. LHCb Collaboration, R. Aaij et al., Measurements of prompt charm production cross-sections in $p p$ collisions at $\sqrt{s}=13$ TeV. JHEP 03, 159 (2016). arXiv:1510.01707

124. ATLAS Collaboration, G. Aad et al., Measurement of $D^{* \pm}, D^{ \pm}$ and $D_{s}^{ \pm}$meson production cross sections in $p p$ collisions at $\sqrt{s}=$ $7 \mathrm{TeV}$ with the ATLAS detector. Nucl. Phys. B 907, 717-763 (2016). arXiv: 1512.02913

125. S. Alekhin, K. Daum, K. Lipka, S. Moch, Determination of the charm-quark mass in the MS-bar scheme using charm production data from deep inelastic scattering at HERA. Phys. Lett. B 718, 550-557 (2012). arXiv:1209.0436 
126. O. Behnke, A. Geiser, M. Lisovyi, Charm, beauty and top at HERA. Prog. Part. Nucl. Phys. 84, 1-72 (2015). arXiv: 1506.07519

127. S. Alekhin, J. Blümlein, K. Daum, K. Lipka, S. Moch, Precise charm-quark mass from deep-inelastic scattering. Phys. Lett. B 720, 172 (2013). arXiv:1212.2355
128. A. Buckley, J. Ferrando, S. Lloyd, K. Nordstrm, B. Page et al., LHAPDF6: parton density access in the LHC precision era. Eur. Phys. J. C 75, 132 (2015). arXiv: 1412.7420 